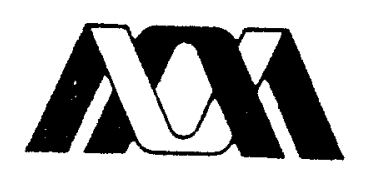

Casa abierta al tiempo

\title{
Uso del Método de Series para el Estudio de Sistemas Cuánticos Confinados
}

Tesis que presenta:

Norberto Aquino Aquino

para la obtención del grado de

Doctor en Ciencias.

Octubre, 1997.

UNIVERSIDAD AUTONOMA METROPOLITANA-IZTAPALAPA

División de Ciencias Básicas e Ingeniería 


\title{
DEDICATORIA
}

Este trabajo está dedicado a mis hijos:

\author{
Norberto y Erandi
}

\section{y}

a la memoria de mis abuelos:

\section{Leoncio Aquino S. y Celestina Gómez}

quienes siempre me motivaron para continuar estudiando. 


\section{AGRADECIMIENTOS}

Deseo expresar mi agradecimiento a los miembros del jurado: Dr. Eugenio Ley-Koo, Dr. Salvador Cruz, Dra. Julia Tagueña, Dr. Eleuterio Castaño y al Dr. Germán Campoy, por sus útiles comentarios y observaciones sobre este trabajo.

Al Dr. Salvador Cruz por haber dirigido esta tesis, así como por sus útiles consejos.

A mi esposa Alejandra por su cariño, la infinita paciencia y comprensión que mostró durante el tiempo en el que se desarrolló este trabajo.

Quiero agradecer también, a mis amigos, cuyos comentarios me motivaron a finalizar este trabajo, en particular a: José Luis Jiménez, Miguel Angel López, Lidia Jiménez, Elizabeth Salinas, Jorge Garza, Rubicelia Vargas, Alberto Vela, José Luis Córdova y a mis amigos de toda la vida Jaime y Javier. 


\section{Contenido}

\section{Dedicatoria}

Agradecimientos

\section{Intròducción}

1 Importancia de los sistemas cuánticos confinados. 1

2 Objetivo de este trabajo. 3

3 Contenido del trabajo. 4

\section{Metodología en el estudio de los Sistemas Cuánticos Confinados}

1.1 Métodos comunmente empleados. 7

1.1.1 Métodos exactos. 7

1.1.2 Método variacional directo. 10

1.1.3 Métodos perturbativos. 11

1.1.4 Método de perturbación de fronteras. 12

1.1.5 Teorema del Virial. 14

1.1.6 Otros métodos. 16

1.2 Método de series. 17

\section{El oscilador armónico isotrópico confinado.}

2.1 Antecedentes. 21

2.2 Eigenvalores de la energía. 22

2.3 Los valores esperados de la posición. 30

2.4 Conclusiones. 33 


\section{Atomo de hidrógeno 3D confinado.}

3.1 Antecedentes. 35

3. $2 \mathrm{El}$ confinamiento dentro de cajas impenetrables. 36

3.2.1 Hidrógeno estudiado en la base del OIAC. 36

3.2.2 El cálculo de energías propias con alta precisión. 39

3.2.3 Valores esperados de potencias de la coordenada radial. 44

3.2.4 Propiedades fisicas. 46

3.2.5 Funciones de onda. 48

3.3 Atomo de $\mathrm{H}$ con condiciones a la frontera tipo Neumann. 50.

$3.4 \mathrm{El}$ átomo de $\mathrm{H}$ dentro de una caja penetrable. 51

3.5 Conclusiones. 53

4. El átomo de hidrógeno $2 \mathrm{D}$ confinado.

4.1 Introducción. 55

4.2 Atomo de hidrógeno libre en 2D. 57

4.3 Atomo de hidrógeno $2 \mathrm{D}$ confinado. 58

4.3.1 Eigenvalores de la energía. 60

4.3.2 Valores esperados de r. 62

4.4 Método de series. 66

4.5 Conclusiones. 67

5. La inversión del $\mathrm{NH}_{3}$ y sus especies isotópicas.

5.1 Introducción. 69

5.2 El potencial anarmónico cuártico. 71

5.3 Método de series. 72

5.4 Espectro de inversión del NH3. 75

5. 5 Especies isotópicas del $\mathrm{NH} 3.77$

5.6 Conclusiones. 78

6 Conclusiones

Conclusiones. 81

Referencias. 86 


\section{Introdución}

\section{Importancia de los sistemas cuánticos confi- nados}

Existen muchos problemas en diversos campos de la física que por diversas razones deben de considerarse como sistemas cuánticos confinados (SCC).

Llamaremos acotado o confinado a un sistema cuántico que esté descrito por una función de onda que satisface determinadas condiciones de frontera (CF) para valores finitos de las coordenadas.

Entre los numerosos modelos estudiados [1-94, 98-99, 102-105] podemos destacar el átomo de hidrógeno confinado por paredes esféricas impenetrables [1-16, $19,21,24,25,29]$, penetrables $[17,23,25]$ y paredes de diversas formas $[18,20]$, el oscilador armónico confinado en una, dos y tres dimensiones [30-50], átomos de muchos electrones y moléculas confinadas dentro de diferentes recintos y ciertas condiciones de frontera [51-58, 92-93]. Estos modelos sirven como base para tratar muy diversos problemas, algunos de los cuales son los siguientes:

a) Materia bajo altas presiones. El comportamiento de una partícula (átomo, molécula, ión, etc) en un medio sometido a muy altas presiones puede estudiarse, en primera aproximación, suponiendo que el efecto de las cargas negativas de las partículas vecinas crean una superficie de potencial infinita. Debido a esto, la función de onda debe anularse sobre esta superficie de potencial. Esto permite definir de manera sencilla a la presión, así como algunas otras propiedades físicas 
tales como la polarizabilidad, el apantallamiento magnético nuclear y el desdoblamiento hiperfino $[1-4,6,12,17,26,29]$. El mismo modelo se ha aplicado al estudio del hidrógeno confinado dentro del cuarzo-alfa [13, 16, 18-20, 26].

Un modelo más realista sugiere la utilización de un potencial finito, que encajona parcialmente al sistema dentro de paredes penetrables [21-25].

b) También se pueden estudiar otras propiedades como el calor específico de metales, transiciones de fase de segundo orden y algunas propiedades magnéticas y eléctricas de los metales mediante el modelo del oscilador en una, dos y tres dimensiones confinado con condiciones de Dirichlet [39-41] o de la partícula en una caja con un potencial que varía en forma lineal [67].

c) Aplicaciones en Astronomía. El modelo del oscilador armónico confinado ha sido muy útil en el estudio de la transformación protón-deuterón como fuente de energía en estrellas densas [33] y en el estudio de la relación fundamental masa-radio en la teoría de las enanas blancas [35] y también el estudio de la velocidad de escape de estrellas de cúmulos globulares o galácticos [32].

d) Aplicación a sistemas libres. Muchos sistemas cuánticos libres pueden resolverse fácilmente si los confinamos dentro de una caja de paredes impenetrables, es decir se imponen artificialmente condiciones de frontera de Dirichlet. En este caso, las energías y eigenfunciones del problema libre se obtienen al hacer que el tamaño de la caja tienda a infinito. Algunos ejemplos son el oscilador anarmónico unidimensional [78-81], el potencial de Morse [81, 102-103], las frecuencias de inversión del $\mathrm{NH}_{3}$ [83-84], la posición y ancho de las resonancias en la interacción de una partícula con un campo radial [70-71].

e) Aplicaciones al campo del estado sólido. En la física del estado sólido existen muchos sistemas que pueden considerarse como acotados; como por ejemplo: electrones y excitones dentro de pozos y puntos cuánticos, electrones en nanoestructuras y centros de color [85-92].

f) Propiedades de los metales. En la teoría de sólidos de Wigner y Seitz [72-73] la función de onda que describe el movimiento del electrón dentro del volumen atómico satisface condiciones de frontera de Neumann sobre la superficie que lo limita [74-77]. 
g) Otras aplicaciones físicas. Existen otros modelos físicos que pueden ser tratados como sistemas cuánticos acotados; como por ejemplo:

- Fórmulas de dispersión de reacciones nucleares [59-60] y predicción de deformaciones nucleares [64].

- El estudio de los electrones de Bloch en un campo eléctrico y el efecto Stark [15].

- Propiedades de las moléculas diatómicas dipolares en un campo eléctrico [61].

- Superconductividad [62].

- Modelos de Cuarks dentro de una caja [104-105].

- El estudio de la densidad de estados de un sistema desordenado en presencia de un campo eléctrico [69].

Las aplicaciones antes mencionadas no son todas las existentes, el propósito de esta sección no es hacer una revisión exahustiva de las aplicaciones de los modelos cuánticos confinados, sino dar una visión general de las aplicaciones que de ellos se han realizado y siguen realizándose. Sin lugar a dudas los sistemas más ampliamente estudiados son el oscilador armónico y el átomo de hidrógeno acotados con condiciones de Dirichlet o de Neumann. Esto es debido fundamentalmente a que ambos sistemas son muy sencillos; sin embargo, tienen todas las características que se espera encontrar en sistemas más complejos. A pesar de la sencillez de estos dos sistemas su solución no es trivial, ya que los eigenvalores de la energía no pueden expresarse de una manera analítica sencilla, y en general es necesario utilizar el cálculo numérico para determinarlos.

\section{Objetivo de este trabajo}

El objetivo de este trabajo es el estudio de algunos sistemas cuánticos acotados o confinados a través de un método exacto. En él la función de onda se desarrolla 
en una serie de potencias en la(s) variable(s) relevante(s), considerando la dependencia explícita de la función de onda en la energía y la posición de la pared de confinamiento; de esta manera el problema de eigenvalores de la ecuación de Schrödinger se reduce al de encontrar los ceros de una función trascendente. La ventaja de este método es que permite determinar simultáneamente los eigenvalores y eigenfunciones del problema con un alto grado de precisión. En los problemas que trataremos sus eigenvalores se determinan con al menos 10 cifras significativas después del punto decimal, además el método es numéricamente estable y es fácil de implantar en cualquier computadora y cada uno de los eigenvalores se obtiene en un tiempo de cómputo muy reducido (menos de 1 segundo en una PC-386 DX a 33Mhz sin coprocesador matemático).

Para ser más precisos, estudiaremos el oscilador isotrópico en tres dimensiones y el átomo de hidrógeno en dos dimensiones confinado mediante paredes impenetrables, el átomo de hidrógeno en tres dimensiones confinado mediante paredes esféricas penetrables e impenetrables y usaremos el método del encajonamiento para el estudio de las frecuencias de inversión del $\mathrm{NH}_{3}$.

Aunque algunos de estos sistemas ya han sido estudiados previamente por muchos autores, estos estudios han sido realizados con métodos que no tienen la precisión del que aquí se propone, por lo que las propiedades físicas que predicen tienen un carácter más cercano a lo cualitativo. En este trabajo obtenemos estos valores con alta precisión para que puedan servir de referencia y poder comparar la eficiencia y precisión de los métodos que en el futuro se propongan para el estudio de los SCC. Por otra parte, la simplicidad y eficiencia del método propuesto no solamente permitirá establecer valores de referencia para otros cálculos, sino el tratamiento de sistemas novedosos que aún no se han explorado.

\section{Contenido del trabajo}

En el capítulo 1 describimos brevemente los métodos más comunmente empleados en el estudio de los sistemas cuánticos confinados, haciendo hincapié en las ventajas y desventajas de cada formulación.

También introducimos el método de las series que usaremos a lo largo de 
este trabajo. Con él estudiaremos cuatro ejemplos para mostrar, con resultados concretos, el poder del método empleado; El oscilador armónico isotrópico en tres dimensiones y el átomo de hidrógeno confinado en dos y tres dimensiones, $\mathrm{y}$ el potencial de doble pozo, responsable de la inversión de la molécula $\mathrm{NH}_{3}$. El objetivo es mostrar que este método permite obtener eigenvalores de alta precisión y simultáneamente, las eigenfunciones del problema.

En el capítulo 2 empleamos el método de las series para estudiar el problema del oscilador armónico isotrópico confinado (OAIC) en tres dimensiones, que se confina dentro de paredes esféricas impenetrables [38]. Calculamos los eigenvalores de la energía para diferentes tamaños de caja, los resultados obtenidos por este método lo comparamos con los valores reportados en la literatura y encontrmos que el método propuesto nos permite obtener valores de alta precisión, que pueden tomarse como referencia para determinar la precisión de otros métodos. Además calculamos algunos de los valores esperados de la posición. Es importante hacer notar que estos valores no se encuentran reportados en la literatura, así como tampoco se han reportado propiedades físicas tales como la presión.

El método de series seleccionado para el estudio proporciona los eigenvalores de la energía con una alta precisión a un costo computacional muy bajo. Los valores obtenidos por el presente método son los más precisos que se pueden encontrar en la literatura [38].

En el capítulo 3 estudiamos el átomo de hidrógeno confinado. Como un ejemplo de las posibles aplicaciones del oscilador armónico isotrópico confinado, estudiado en el capítulo 2, se resuelve el problema del átomo de hidrógeno confinado dentro de una caja esférica de paredes impenetrables, empleando la base del OAIC [38] encontrándose que este procedimiento proporciona energías satisfactorias para el estado base y algunos estados excitados. Siguiendo el mismo razonamiento empleado en el capítulo 2, volvemos a emplear el método de las series para estudiar el problema del átomo de hidrógeno confinado, dentro de paredes penetrables [29] e impenetrables. Los resultados obtenidos poseen una precisión que rebasa por varios ordenes de magnitud a la de los cálculos reportados anteriormente en la literatura. En este capítulo calculamos los eigenvalores de la energía, algunos momentos esperados de la posición, la presión, la estructura 
hiperfina, el apantallamiento magnético nuclear y la polarizabilidad de átomo de hidrógeno como función del tamaño de la caja de confinamiento [29]. Además resolvemos el problema del átomo de hidrógeno bajo condiciones de frontera de Neumann. Todos estos cálculos se realizaron con el mismo método de series a un costo computacional muy bajo.

En el capítulo 4 se estudia el átomo de hidrógeno en dos dimensiones confinado mediante paredes impenetrables. En este capítulo hacemos uso del método variacional directo para el cálculo de las energías, las cuales se comparan con las que se obtienen mediante el método de las series que hemos empleado en capitulos anteriores. También calculamos algunos de los valores esperados de la posición. Además se discuten las analogías y diferencias entre los sistemas (libres y confinados) en dos y tres dimensiones. El estudio del átomo de hidrógeno bidimensional confinado, es un tópico que no se encuentra discutido en la literatura. Sin embargo, este modelo puede ser de utilidad para entender fenómenos asociados con puntos cuánticos bidimensionales. Los resultados obtenidos mediante el método de las series constituyen la primera referencia del cálculo preciso de este sistema.

En el capítulo 5 se usa el método de las series para encontrar los eigenvalores del potencial de doble pozo, que aparece en el problema de la inversión del $\mathrm{NH}_{3}$, y sus especies isotópicas [84]. Para verificar la precisión de los eigenvalores obtenidos por el método de las series, éstos se calculan mediante un método independiente [158] basado en las propiedades asintóticas de las funciones de onda. Encontramos que ambos métodos predicen los mismos resultados, confirmando que el método de las series produce resultados de muy alta calidad.

Las frecuencias de inversión obtenidas para el $\mathrm{NH}_{3}$ y sus especies isotópicas se comparan con las reportadas en la literatura, las que han sido predichas con otros modelos y con los valores experimentales, encontrándose que el modelo que aquí proponemos proporciona los mejores valores que se han reportado hasta el momento.

Finalmente en el capítulo 6, se dan las conclusiones de este trabajo y se sugieren algunos sistemas para los cuales el confinamiento es importante y que pueden ser estudiados, de manera cualitativa y cuantitativa precisa utilizando el método empleado en este trabajo. 


\section{Capítulo 1}

\section{Metodología en el estudio de los Sistemas Cuánticos Confinados}

Como ya lo mencionamos, en la gran mayoría de sistemas cuánticos confinados no es posible resolver la ecuación de Schrödinger en forma analítica y por lo tanto tampoco se tienen expresiones analíticas para las eigenfunciones y los eigenvalores. No obstante, siempre se pueden encontrar soluciones aproximadas, cuya precisión varía dependiendo del método de aproximación seleccionado. En este capítulo describiremos brevemente los métodos más comunmente empleados en el estudio de los SCC, y propondremos un método alternativo que produce eigenvalores y eigenfunciones de alta precisión.

\subsection{Métodos comunmente empleados}

\subsubsection{Métodos exactos}

Estos métodos están basados en el desarollo de la función de onda en una serie de potencias en la coordenada relevante y su posterior sustitución en la ecuación de Schrödinger, con el fin de determinar una relación de recurrencia entre los coeficientes de la serie. Para ilustrar este método tomaremos a manera de ejemplo, 
el caso del átomo de hidrógeno confinado por paredes esféricas impenetrables. El hamiltoniano de este sistema (en unidades atómicas) se puede escribir como:

$$
H=-\frac{1}{2} \nabla^{2}-1 / r+U(r)
$$

el potencial de confinamiento $U(r)$ esta dado por

$$
U(r)=\left\{\begin{array}{cc}
+\infty, & r>r_{0} \\
0, & r \leq r_{0}
\end{array}\right.
$$

donde $r_{0}$ es el radio de la caja esférica. El problema es el de un potencial central, y posee simetría esférica por lo que la función de onda total se puede escribir como:

$$
\psi(r, \theta, \phi)=N R(r) Y_{l}^{m}(r, \theta, \phi)
$$

donde $Y_{l}^{m}(r, \theta, \phi)$ son los armónicos esféricos, $N$ es la constante de normalización de la función de onda y $R(r)$ es la parte radial de la función de onda, que satisface la ecuación

$$
\left(\frac{d^{2}}{d r^{2}}+\frac{2}{r} \frac{d}{d r}-\frac{l(l+1)}{r^{2}}+\frac{2}{r}+2 E\right) R(r)=0,
$$

aquí $E$ representa la energía del sistema. Dado que el potencial de confinamiento tiene barreras impenetrables, la función de onda debe de anularse en la superficie de la caja, es decir:

$$
\psi\left(r=r_{0}, \theta, \phi\right)=0,
$$

condición que es equivalente a:

$$
R\left(r_{0}\right)=0
$$


Haciendo la sustitución $R(r)=r^{l} f(r)$, la ecuación de Schrödinger radial (4) se transforma en

$$
\left(\frac{d^{2}}{d r^{2}}+\frac{2(l+1)}{r} \frac{d}{d r}+\frac{2}{r}+2 E\right) f(r)=0
$$

Proponemos que la solución $f(r)$ este dada por la siguiente serie

$$
f(r)=\sum_{k=0}^{\infty} a_{k} r^{k}
$$

Al sustituir esta solución en la ecuación (6) se encuentra la relación de recurrencia

$$
a_{k+2}=-2\left(E a_{k}+a_{k+1}\right) /(k+2)(k+2 l+3) .
$$

Asi que la condición de frontera (5) se puede escribir como

$$
R\left(r_{0}\right)=\sum_{k=0}^{\infty} a_{k} r^{k}=0
$$

Las raíces de esta ecuación son los valores propios de las energías del sistema. En la práctica se toma un número finito de términos para aproximar el valor de la serie, dependiendo del grado de precisión con la cual se deseen encontrar los eigenvalores de la energía. Cuando el valor de $r_{0}$ crece, se hace necesario tomar un mayor número de términos y mantener la precisión deseada en los eigenvalores. Esto impone un límite para el tamaño de las cajas en las cuales este método se puede aplicar. Para evaluar por ejemplo los valores esperados, de la posición se deben de evaluar los coeficientes $a_{k}$ para cada valor de $r_{0}$ y después hacer las integraciones necesarias. La primera solución al problema del átomo de hidrógeno confinado por paredes impenetrables usando un método de esta clase fue dada por Ley-Koo y Rubinstein [17].

De entre los problemas que se han resuelto mediante esta clase de métodos podemos mencionar el átomo de hidrógeno confinado dentro de cajas con paredes 
penetrables e impenetrables [2-4,17], el oscilador armónico en una y tres dimensiones dentro de paredes impentrables [44, 49], algunos iones moleculares en cajas de diversas geometrías $[18,20]$, centros $\mathrm{F}$ en halogenuros alcalinos [106], y también en electrones balísticos en nano-estructuras [107].

\subsubsection{Método variacional directo}

Aun cuando no sea posible encontrar soluciones analíticas a muchos sistemas cuánticos, siempre se puede dar una solución aproximada. Uno de los primeros métodos que se emplearon para encontrar soluciones aproximadas y que aun hoy es uno de los más usados es el método variacional. Para sistemas cuánticos confinados Marín y Cruz [24-26] propusieron el método variacional directo, que consiste en emplear una función de prueba de la siguiente forma:

$$
\Psi\left(\vec{r}, \alpha_{i}\right)=\psi\left(\vec{r}, \alpha_{i}\right) \chi(\vec{r}),
$$

donde $\operatorname{los} \alpha_{i}$ son un conjunto de parámetros variacionales, mientras que $\psi$ es la solución de la ecuación de Schrödinger para el problema libre, en tanto que que $\chi$ es una función de corte bien comportada, que se anula exactamente en la frontera del sistema (para paredes impenetrables), haciendo que la función de onda $\Psi$ satisfaga también la condición de frontera del problema confinado. Con esta función de prueba (ec. 10) se minimiza el valor esperado de la energía

$$
E\left(\alpha_{i}\right)=\frac{\langle\Psi|H| \Psi\rangle}{\langle\Psi \mid \Psi\rangle},
$$

respecto a los parámetros variacionales

$$
\frac{\partial E}{\partial \alpha_{i}}=0,
$$

para obtener la energía y la función de onda óptimas. En la ecuación (11a) y (11b), $E$ representa el valor esperado de la energía y $H$ al hamiltoniano del 
sistema. Debe notarse que la información sobre la geometría del sistema esta contenida en la función $\chi$, y que además esta función no es única.

Con este método se han tratado los problemas del átomo de hidrógeno confinado por paredes impenetrables y penetrables [26], el átomo de hidrógeno confinado fuera del centro de una esfera impenetrable [156], el oscilador armónico en una y tres dimensiones, y el átomo de helio confinado por una caja esférica $[25,26,49]$.

\subsubsection{Métodos perturbativos}

Otro método aproximado que se usa con mucha frecuencia en mécanica cuántica es el de teoría de perturbaciones [108] , en particular perturbaciones independientes del tiempo. La teoría es bien conocida y se le puede consultar en cualquier libro de texto moderno de mécanica cuántica [108]. En esta sección solo mencionaremos algunos detalles importantes para su aplicación en sistemas confinados. La idea general es que tenemos un hamiltoniano $H$ para nuestro sistema, y este se puede escribir como:

$$
H=H_{0}+U,
$$

donde $H_{0}$ es el hamiltoniano del sistema no perturbado, el cual tiene una solución conocida y por lo tanto conocemos los eigevalores $E_{n}$ y eigenfunciones $\psi_{n}$. Si la perturbación $U$ es lo suficientemente pequeña, se puede emplear teoría de perturbaciones y calcular la corrección de la energía a algún orden determinado. El problema en su aplicación es que sólo para algunos cuantos problemas confinados se conoce el espectro de energías y sus correspondientes eigenfunciones, lo cual hace poco práctico al método.

El método se puede aplicar sólo en situaciones extremas, esto es, cuando la región de confinamiento es muy pequeña o cuando es muy grande. En el caso de que la región de confinamiento sea muy pequeña, la energía cinética de la partícula es más grande que la energía potencial y por lo tanto podemos utilizar como funciones no perturbadas a la base de funciones de onda de una partícula en 
una caja impenetrable y como perturbación al potencial debido a todas las otras interacciones. En el otro caso, cuando la región de confinamiento es muy grande,

- tenemos un problema muy cercano al caso libre y su diferencia de las energías respecto al sistema libre será muy pequeña, así que el cambio introducido por el confinamiento podrá tratarse como una perturbación al sistema libre. Las funciones de onda no pertubadas en este caso serán las del sistema libre adaptadas para que satisfagan las condiciones de frontera.

Por ejemplo, para un átomo hidrogenoide confinado dentro de una caja esférica impenetrable de radio $r_{0}$ pequeño, se toma a las funciones de onda de una partícula dentro de una caja esférica similar, como las funciones no perturbadas.

Este enfoque ha sido empleado por diversos autores para estudiar algunas propiedades del hidrógeno a altas presiones [12] y en el estudio de los niveles de energía de excitones en esferas semiconductoras o puntos cuánticos [109-110].

\subsubsection{Método de perturbación de fronteras}

El presente método fue originalmente desarrollado por Frölich [74] y posteriormente modificado por Singh [54] en su estudio del efecto de la presión sobre el ión molecular de hidrógeno $H_{2}^{+}$. La versión moderna es debida a Gorecki y ByersBrown [111-112], quienes emplearon en el estudio de sistemas confinados tanto penetrables como impenetrables. Mediante este método se han tratado también al oscilador armónico y al átomo de hidrógeno confinados en cajas muy grandes.

El método esencialmente es el siguiente. Consideremos un sistema sin confinamiento, cuyo hamiltoniano es

$$
H_{0}=-\frac{1}{2} \nabla^{2}+V(r)
$$

La ecuación de Schrödinger correspondiente es

$$
H_{0} \phi_{0}=E_{0} \phi_{0}
$$


Ahora supongamos que confinamos al sistema anterior dentro de una región $D$ impenetrable con una frontera $S$, así que, el nuevo hamiltoniano será:

$$
H=-\frac{1}{2} \nabla^{2}+V(\vec{r})+U(\vec{r})
$$

Donde $U(\vec{r})$ es el potencial de confinamiento, que está dado por

$$
U(\vec{r})=\left\{\begin{array}{cc}
+\infty, & \vec{r} \notin D \\
0, & \vec{r} \in D .
\end{array}\right.
$$

La ecuación de Schrödinger correspondiente al hamiltoniano $H$ (ec. 15) se puede escribir como

$$
H \phi=\left(-\frac{1}{2} \nabla^{2}+V(\vec{r})\right) \phi=E \phi ; \vec{r} \in D
$$

con la condición de frontera $\phi(\vec{r})=0$ si $\vec{r} \in S$.

Ahora multipliquemos la ecuación (14) por $\phi$ y la (17) por $\phi_{0}$ y restándolas, se obtiene

$$
-\frac{1}{2}\left(\phi \nabla^{2} \phi_{0}-\phi_{0} \nabla^{2} \phi\right)=\left(E-E_{0}\right) \phi \phi_{0}=\Delta E \phi \phi_{0}
$$

donde $\Delta E=E-E_{0}$, representa el cambio de energía debido al confinamiento. Como la función de onda satisface la condición de frontera $\phi(\vec{r})=0$ para $\vec{r} \in S$, se propone que $\phi$ tenga la forma

$$
\phi(\vec{r})=\phi_{0}(\vec{r}) f(\vec{r})
$$

en la cual la función $f$ es no singular y satisface la condición de frontera $f(\vec{r})=0$ cuando $\vec{r} \in S$. Al sustituir la ecuación (19) en la ec. (18) e integrar sobre el volumen encerrado por $S$, se obtiene 


$$
\Delta E=\frac{\int \phi_{0} \nabla f \cdot \nabla \phi_{0} d \tau+\frac{1}{2} \int \phi_{0} \nabla^{2} f d \tau}{\int f \phi^{2} d \tau}
$$

Para determinar el valor de $\Delta E$, es necesario conocer la función $f$. En este método se realiza una optimización de $\Delta E$ con respecto al conjunto de funciones $f$ que satisfacen la condición de frontera $f(\vec{r})=0$ en la superficie $S$ y que minimiza la ec. (20) con respecto a los contornos de $D$ representados por $S(f)=$ constante. Usualmente este procedimiento lleva a una ecuación integral con valores propios $\Delta E$ y vectores propios $f$ respectivamente.

Con este método se han encontrado expresiones analíticas para los eigenvalores de la energía del oscilador armónico [7,39] y el átomo de hidrógeno confinados, válidas cuando el radio de la caja que los confina es muy grande.

Los resultados que se obtienen con este método en general son buenos, aunque es muy laborioso y por lo tanto costoso desde el punto de vista del tiempo de cálculo en una computadora.

\subsubsection{Teorema del Virial}

Si analizamos la rapidez de cambio en el tiempo del valor esperado del operador A, que no depende explícitamente del tiempo, se puede demostrar que

$$
\frac{d<A>}{d t}=\frac{1}{i \hbar}\langle[A, H]\rangle,
$$

donde $H$ es el hamiltoniano del sistema. En particular, para el operador $\vec{r} \cdot \vec{p}$, se encuentra que:

$$
\frac{d}{d t}<\vec{r} \cdot \vec{p}>=0,
$$

si además $H=\frac{p^{2}}{2 m}+V$, entonces [108] 


$$
2<T>=<\vec{r} \cdot \nabla V(r)>
$$

Si $V$ es esféricamente simétrico y proporcional a $r^{n}$, el valor esperado existe. De la ecuación (23) se encuentra que

$$
2<T>=n<V>
$$

Para el potencial Coulombiano $n=-1$, así que $2\langle T\rangle=-\langle V\rangle$, mientras que para el oscilador armónico $n=2$, de donde se tiene que $\langle T\rangle=\langle V\rangle$.

El teorema del Virial es muy útil en el estudio de sistemas cuánticos libres, ya que mediante el uso de transformaciones de escala y este teorema, es posible reducir en uno el número de parámetros variacionales a optimizar. En el caso de los sistemas cuánticos confinados, las integrales involucradas en el cálculo del valor medio de la energía no se calculan en el intervalo sobre todo el espacio, sino solo en una región restringida del mismo, por lo cual las transformaciones de escala ya no son tan útiles. Así también debe notarse que con el confinamiento espacial el teorema del Virial (ec. 23) debe de modificarse. Para un sistema confinado este se puede escribir como [98]:

$$
2<T>-<\vec{r} \cdot \nabla V(r)>=F\left(E^{\prime}, q_{0}\right)
$$

donde $q_{0}$ representa a la(s) coordenada(s) que define(n) la frontera de la región de confinamiento, $E^{\prime}$ es la variación de la energía respecto a $q_{0}, F$ es una función que depende tanto del sistema en estudio como de la forma y la altura de la(s) barrera(s) de confinamiento. En el caso libre $E^{\prime}=0, q_{0}=\infty, F=0$.

Para potenciales centrales y fronteras esféricas e impenetrables [113-116] se sabe que:

$$
2<T>-<\vec{r} \cdot \nabla V(r)>=-r_{0} \frac{\partial E}{\partial r_{0}}
$$


donde $r_{0}$ es el radio de la esfera de confinamiento, mientras que $E$ es la energía del sistema.

Mediante la ecuación (26) podemos calcular la presión que las fronteras esféricas ejercen sobre el sistema, ya que

$$
P=\frac{1}{4 \pi r_{0}^{2}} \frac{\partial E}{\partial r_{0}}=\frac{1}{4 \pi r_{0}^{3}}(2<T>-<\vec{r} \cdot \nabla V(r)>) .
$$

La validez de la ec. (26) para potenciales seccionalmente definidos, como en el caso de fronteras penetrables, se encuentra aun a discusión [116], mientras que para el caso impentrable se tiene una demostración rigurosa de ella [113-155].

En la mayoría de los casos no se conocen las soluciones exactas de la ecuación de Schrödinger para sistemas confinados, así que para calcular la presión que las fronteras ejercen sobre el sistema, se usan las funciones de onda que provee un método aproximado, como por ejemplo, el variacional.

\subsubsection{Otros métodos}

A continuación mencionamos algunos otros métodos que se han empleado en menor medida en el estudio de los sistemas cuánticos confinados:

- El método WKB. Este método proporciona aproximaciones analíticas para los eigenvalores, sin embargo estos valores no son muy precisos, en particular para los estados más bajos. Pese a su amplia aplicación en sistemas libre, sólo se ha empleado en el estudio del átomo de hidrógeno y el oscilador armónico confinados $[4,44]$.

- Método de los elementos finitos. Este método proporciona eigenvalores de la energía en forma numérica y valores de la función de onda sobre una malla. La precisión de los cálculos es razonablemente buena. Mediante este método se han estudiado el átomo de hidrógeno confinado [14] y en presencia de un campo eléctrico uniforme [15]. 
- Método de diferencias finitas. Este método al igual que el anterior es completamente numéricos y proporciona buenos valores para las energías del sistema. Este método se ha empleado en el estudio del oscilador armónico y anarmónico acotados [30].

- Método de Hartree-Fock. El método de Hartree-Fock se ha empleado en el estudio del átomo de hidrógeno, el átomo de helio e iones de dos electrones, $\mathrm{Li}, \mathrm{Be}, \mathrm{B}, \mathrm{C}$ y $\mathrm{Ne}$ dentro de cajas esféricas impenetrables [57, 58]. Este es un método comunmente empleado en el estudio de átomos y moléculas libres, con la variante de que ahora los orbitales del caso confinado deben de cumplir la condición de frontera sobre la superficie de la caja esférica. Estos se construyen de la siguiente manera. Se multiplica al orbital del sistema libre por una función de corte que se anula sobre la frontera del sistema. Los resultados de este cálculo son razonablemente buenos, pero si se emplea el método CI [58] los resultados son excelentes. La desventaja de este tipo de cálculos es la gran cantidad de tiempo de cómputo consumido.

- Método de Monte Carlo Cuántico. Este método es considerado por algunos como un método exacto por la gran precisión que se obtiene en los eigenvalores, aunque su uso se ha restringido solo al átomo de hidrógeno, al helio [27] y a la molécula de $H_{2}$ confinada en cajas esferoidales impenetrables $[117]$.

\subsection{Método de series}

El método que emplearemos para resolver la ecuación de Schrödinger en este trabajo, fue originalmente introducido por Campoy y Palma [78-80] para resolver sistemas cuánticos libres, mientras que Aquino [29, 38] lo generalizó para mostrar que también podía emplearse para resolver problemas confinados por paredes impenetrables.

El método propuesto por Campoy y Palma [78-80] para sistemas libres lo describiremos a continuación. La ecuación de Schrödinger independiente del tiempo, 
para un potencial unidimensional arbitrario $V(x)$, que en unidades atómicas es:

$$
\psi^{\prime \prime}=2[V(x)-\epsilon] \psi
$$

donde $\epsilon$ representa el eigenvalor de la energía y $\psi$ la función de onda. Una de las principales características del método es la de tratar a la función de onda $\psi$ como una función de la posición y de la energía, es decir:

$$
\psi=\psi(x, \epsilon)
$$

de tal suerte que la derivada de la ecuación (1) con respecto a la energía es entonces

$$
\partial \psi^{\prime \prime} / \partial \epsilon=2[V(x)-\epsilon](\partial \psi / \partial \epsilon)-2 \psi
$$

De esta ecuación se puede obtener una relación de recurrencia para cada una de las derivadas y con ello reconstruir la función de onda. El problema es que no se conoce el valor de la energía $\epsilon$. Para poder determinar este valor Campoy y Palma imponen la siguiente restricción sobre las funciones de onda: la función de onda debe anularse para valores de $x$ "razonablemente grandes", por ejemplo, para valores de $x$ que sean una longitud de onda más grandes que el punto de retorno clásico. Como $\epsilon$ es una incógnita, se le da un valor inicial estimativo, se selecciona un valor $x_{0}$ como se mencionó antes, y se modifica el valor inicialmente propuesto de $\epsilon$, hasta que la función de onda (29) se anule en $x_{0}$. Una vez obtenido el valor de $\epsilon$ con la precisión requerida, se incrementa el valor de $x_{0}$ en una cantidad pequeña $\delta$ y se calcula el nuevo valor de $\epsilon$ para el cual la función (29) se anula en $x_{0}+\delta$. Después de algunos incrementos en $x_{0}$ se obtiene el valor de $\epsilon$ para el sistema libre. En este procedimiento los valores intermedios de $\epsilon$ no tienen significado físico, son simplemente pasos auxiliares para obter el valor de $\epsilon$ en $x_{0}$ suficientemente grande.

Para poder calcular los eigenvalores de la energía y las eigenfunciones de los SCC, Aquino [29,38] observó que la restricción impuesta por Campoy y Palma sobre 
la función de onda no es necesaria para un sistema confinado por una barrera infinita, ya que la función de onda se anula en la posición de la barrera y fuera de ella, y la barrera puede estar en cualquier posición $x_{0}$, grande o pequeña. De esta manera también le dió un significado físico a los estados intermedios que Campoy y Palma encontraron en sus cálculos. Estos son los estados de un sistema cuántico confinado. Además el valor de $\epsilon$ para el sistema libre se obtiene cuando $x_{0}$ se hace muy grande.

En un sistema cuántico confinado mediante una barrera impenetrable en el punto $x_{0}$, buscamos la energía $\epsilon_{e}$ para cual se satisface la siguiente relación:

$$
\psi\left(x_{0}, \epsilon_{e}\right) \equiv 0
$$

Entonces, la determinación de los eigenvalores de la ecuación (28) se reduce a encontrar los ceros de la función $\psi$ en $x_{0}$. Un procedimiento es el siguiente: se da un valor inicial (de prueba) de $\epsilon_{i}$ y se resuelven numéricamente las ecuaciones (28) y $(30)$ para $\psi$ y $\partial \psi / \partial \epsilon$. Con esto obtenemos $\psi\left(x_{0}, \epsilon_{i}\right)$ y $\partial \psi\left(x_{0}, \epsilon_{i}\right) / \partial \epsilon$, y entonces, se calcula la corrección para la energía mediante el método de Newton-Raphson [95-97],

$$
\epsilon_{i+1}=\epsilon_{i}-\psi\left(x_{0}, \epsilon_{i}\right) /\left[\partial \psi\left(x_{0}, \epsilon_{i}\right) / \partial \epsilon\right]
$$

Con este nuevo valor de la energía iteramos las ecuaciones (28) y (30) hasta obtener el eigenvalor $\epsilon_{f}$ con la precisión requerida.

En este trabajo representaremos a la función $\psi$ mediante una serie de Taylor alrededor del punto $x=0$, que es donde se conocen tanto el valor de la función de onda como el de algunas de sus primeras derivadas. Veremos en los capítulos 2 y 3 como se aplica el método en cada problema, lo que nos hará tener una idea más clara de como usarlo en un problema más complicado.

La ventaja de este método sobre los anteriores es el bajo costo computacional, ya que el cálculo de los eigenvalores y eigenfunciones se puede realizar en cualquier computadora, e incluso en una calculadora programable. El método también es 
numéricamente estable. Con él se han conseguido los mejores valores reportados de las energías, para el oscilador armónico isotrópico tridimensinal [38] y para el átomo de hidrógeno confinados [29] mediante paredes esféricas impenetrables. 


\section{Capítulo 2}

\section{El oscilador armónico isotrópico confinado}

\subsection{Antecedentes}

Como ya lo mencionamos en la introducción de este trabajo, el estudio del oscilador armónico confinado es importante debido a dos razones principalmente. Primeramente tiene un gran número de aplicaciones en diversos campos de la física y además es un sistema en el cual es posible probar de manera directa un buen número de métodos matemáticos. Por estas razones enfocaremos nuestra atención al estudio de este sistema en el presente capítulo.

Es bien sabido que sistemas tan sencillos como el átomo de hidrógeno y el oscilador armónico libres permiten soluciones analíticas de la ecuación de Schrödinger. Sin embargo, cuando estos sistemas cuánticos son confinados por paredes esféricas impenetrables, las soluciones de la ecuación de Schrödinger no son triviales y los eigenvalores se encuentran a través de una ecuación trascendente, que debe resolverse numéricamente.

En la literatura existen reportados estudios sobre el oscilador armónico confinado en 1, 2 y 3 dimensiones [24, 32-35, 40, 41, 48, 94]. En este capítulo nos 
dedicaremos a estudiar exclusivamente el oscilador armónico isotrópico en tres dimensiones confinado en cajas esféricas. Un estudio independiente sobre este último sistema fue realizado por Marín y Cruz [24] y por Fernández y Castro [94], quienes usaron, respectivamente, el método variacional directo, y una combinación de Teoría de pertubaciones con el teorema hipervirial. Los resultados de este último método son en general más precisos que los obtenidos por Marín y Cruz [24]; sin embargo, cuando el radio de la caja aumenta sus resultados pierden precisión.

En este capítulo mostraremos como se pueden obtener las eigenfunciones y los eigenvalores de la energía con una precisión en principio arbitraria para cualquier valor del radio de la caja. Además obtenemos algunos valores esperados de la posición radial, cabe senãlar que hasta el momento no los hemos encuentrado reportados en la literatura.

\subsection{Eigenvalores de la energía}

El hamiltoniano del oscilador armónico isotrópico confinado (OAIC) en unidades naturales (la unidad de la distancia es $(\hbar / m \omega)^{1 / 2}$, mientras que la de la energía es $\hbar \omega$ ) está dado por

$$
H=-\frac{1}{2} \nabla^{2}+r^{2} / 2+U(r)
$$

donde

$$
U(r)=\left\{\begin{array}{cc}
+\infty, & r \geq r_{0} \\
0, & r \leq r_{0}
\end{array}\right.
$$

y $r_{0}$ es el radio de la caja esférica. Ya que el problema es separable, sólo necesitamos resolver la ecuación radial, pues las soluciones angulares en términos de armónicos esféricos son bien conocidas. 
El método descrito en la sección 1.2 fue originalmente desarrollado para potenciales polinomiales, sin embargo, podemos aplicarlo al problema del OAIC si escribimos la ecuación de Schrödinger radial de la siguiente manera [38]:

$$
r^{2} R^{\prime \prime}=-2 r R^{\prime}+l(l+1) R+r^{4} R-2 \epsilon r^{2} R
$$

donde $l$ es el momento angular de la partícula.

A continuación se hace un desarrollo en serie de Taylor para la función de onda $R$, alrededor de $r=0$, donde se conoce el valor inicial de la función.

$$
R(r)=\sum_{k} T_{k}(r)
$$

donde

$$
T_{k}=\left(R^{(k)}(0) / k !\right) r^{k},
$$

la cual se puede evaluar de una manera sencilla. Para ello tomemos la derivada de orden $p$ de la ecuación (3) respecto a $r$, con lo que obtenemos:

$$
\left[r^{2} R^{(2)}\right]^{(p)}=-2\left[r \psi^{(1)}\right]^{(p)}+l(l+1) R^{(p)}-\left[r^{4} R\right]^{(p)}-2 \epsilon\left[r^{2} R\right]^{(p)}
$$

donde las cantidades dentro del paréntesis del super-índice indican el orden de la derivada. Usando ahora la fórmula para la derivada de un producto, junto con las ecuaciones $(4 \mathrm{a})$ y $(4 \mathrm{~b})$, obtenemos finalmente:

$$
T_{p}=r^{2}\left[r^{2} T_{p-4}-2 \epsilon T_{p-2}\right] /[p(p+1)-l(l+1)] .
$$

La función de onda puede evaluarse directamente usando (4a), la ecuación es válida para cualquier valor de $r$ en el intervalo $\left[0, r_{0}\right]$. De esta misma ecuación también se obtiene una expresión para evaluar las derivadas de la función de onda respecto a la energía: 


$$
\partial R / \partial \epsilon=\sum_{m} \partial T_{m} / \partial \epsilon=\sum_{p} \dot{T}_{p}
$$

donde $\dot{T}_{p}$ denota la derivada de $T_{p}$ respecto a la energía. De la ecuación (6), obtenemos la relación de recurrencia:

$$
\dot{T}_{p}=r^{2}\left[r^{2} \dot{T}_{p-4}-2 \epsilon \dot{T}_{p-2}-2 T_{p-2}\right] /[p(p+1)-l(l+1)] .
$$

De las relaciones (4a), (7) y la ec. (32) del capítulo 1, podemos construir cualquier eigenfunción, usando las condiciones iniciales. Para $l=0$ (estados $s$ ), es suficiente con hacer $R_{n}(0)=1$, y de (6) se obtiene que $R_{n}^{\prime}(0)=-1$. Para $l=1$ (estados $p$ ), damos $R_{n}(0)=0$ y $R_{n}^{\prime}(0)=1$; mientras que para $l=2$ (estados $d$ ), debemos dar $R_{n}(0)=0, R_{n}^{\prime}(0)=0, R_{n}^{\prime \prime}(0)=1$.

Una vez que se han obtenido los eigenvalores de la energía $\epsilon_{n}$ podemos calcular los coeficientes del desarrollo de la función de onda sustituyendo los valores de $\epsilon_{n}$ en la ecuación (6) y evaluando $T_{p}$ en $r=1$. Así se obtiene la siguiente expresión para la función de onda:

$$
R_{n}(r)=\sum_{m} T_{m}\left(r=1, \epsilon_{n}\right) r^{m}
$$

La principal ventaja de este método es que el cálculo de los eigenvalores de la energía son muy rápidos y precisos. Cada eigenvalor de la energía se obtuvo con la precisión reportada en la Tabla1, en un tiempo menor a 1 segundo en una microcomputadora 386-DX sin procesador matemático. Debido a la alta precisión con la que se determinan los eigenvalores de la energía por este método, los hemos tomado como referencia para establecer la confiabilidad de otros métodos. Por ejemplo, en la Tabla 1 se muestran los valores para el estado base y el primer estado excitado en función del radio de la caja impenetrable $r_{0}$ y se comparan los resultados obtenidos mediante este método y los obtenidos por Marín y Cruz [24] y Fernández y Castro [94]. Fernández y Castro sólo reportan los valores del estado base, mientras que Marín y Cruz calculan los valores para el primer 
estado excitado también. Los resultados obtenidos por Fernández y Castro tienen buena precisión sólo para valores pequeños de $r_{0}$ y pierden precisión para valores mayores de 2.0 unidades de distancia. Los resultados de Marín y Cruz son menos precisos que los obtenidos por Fernández y Castro, pero el método es muy simple y puede emplearse para calcular los eigenvalores de estados excitados más altos. En la cuarta columna de la Tabla 1 también presentamos los resultados obtenidos usando el método de Ley-Koo y Rubinstein [17]. Aunque este método puede producir resultados con buena precisión, el procedimiento es más complicado que el que hemos mostrado en esta sección.

Tabla 1. Eigenvalores de la energía $\epsilon\left(r_{0}\right)$ para el oscilador isotrópico tridimensional confinado en función del radio de la caja $\left(r_{0}\right)$. Las energías están en unidades de $\hbar \omega$ y el radio en unidades de $(\hbar / m \omega)^{1 / 2}$.

\begin{tabular}{|c|c|c|c|c|}
\hline$r_{0}$ & $\epsilon[24]$ & $\epsilon[94]$ & $\epsilon[17]$ & Presente \\
\hline & Estado & base & $n=0, l=0$ & \\
0.5 & & 19.774534 & & 19.7745341792 \\
1.0 & 5.1313 & 5.0755820 & 5.0755 & 5.0755820152 \\
$(3 / 2)^{1 / 2}$ & & 3.5000000 & & 3.5000000000 \\
1.5 & 2.5265 & 2.5049761 & 2.5050 & 2.5049761785 \\
2.0 & 1.7739 & 1.7648087 & 1.7648 & 1.7648164387 \\
2.5 & 1.5567 & 1.55166 & 1.5514 & 1.5514216545 \\
3.0 & 1.5105 & & 1.5061 & 1.5060815272 \\
4.0 & 1.5033 & & 1.5000 & 1.5000146030 \\
5.0 & 1.5025 & & 1.5000 & 1.5000000036 \\
& & & & \\
\hline & Primer & estado & excitado & $n=0, l=1$ \\
\hline & & & & \\
1.0 & 10.3188 & & 10.2822 & 10.2822569390 \\
1.5 & 4.9169 & & 4.9036 & 4.9035904194 \\
2.0 & 3.2514 & & 3.2469 & 3.2469470987 \\
2.5 & 2.6901 & & 2.6881 & 2.6881439638 \\
3.0 & 2.5337 & & 2.5313 & 2.5312924666 \\
4.0 & 2.5015 & & 2.5001 & 2.5001437781 \\
5.0 & 2.5012 & & 2.5000 & 2.5000000584 \\
\hline
\end{tabular}

Como mencionamos antes, con el presente método también se tiene la ventaja de calcular en forma rápida y precisa los estados excitados. Es sabido que el 


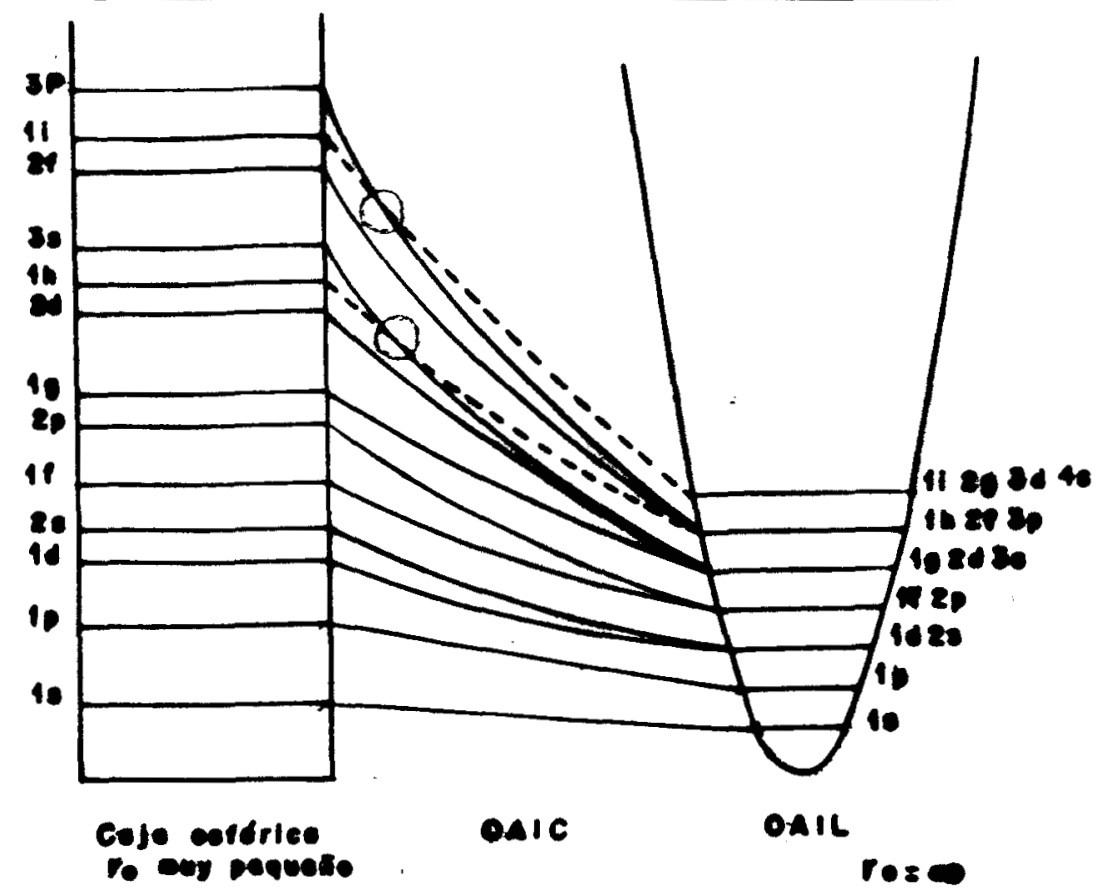

Figura 2.1: Este diagrama muestra la correspondencia de los niveles de energía del OAIC con los de la caja esférica y los del OAIL. Para valores muy pequeños de $r_{0}$ los niveles de energía del OAIC están muy cerca de los de la caja esférica, mientras que para valores grandes de $r_{0}$ son prácticamente los del OAIL. También se muestran dos de los cruces de los niveles de energía.

oscilador armónico isotrópico libre (OAIL) en tres dimensiones, tiene un espectro de energías degenerado, sus eigenvalores son $E=(N+3 / 2) \hbar \omega$ donde $N=2 n+l$, siendo $n$ el número cuántico principal y $l$ el del momento angular. Cuando este sistema (OAIL) se confina dentro de una caja esférica de paredes impenetrables, la degeneración en la energía se rompe, por lo que es conveniente etiquetar a los eigenvalores de la energía del sistema confinado como $E_{n l}\left(r_{0}\right)$.

Los resultados obtenidos mediante el método propuesto en este trabajo para varios estados excitados, se muestran en la Tabla 2, en donde se ha incluído el estado base por completez. Las energías se calcularon para diferentes valores de $n$ y $l$ como funciones del radio de confinamiento $r_{0}$.

Nótese el rompimiento de la degeneracón para las energías $E_{02}\left(r_{0}\right)$ y $E_{10}\left(r_{0}\right)$, asi mismo, para valores pequeños de $r_{0}$ el espectro es no degenerado, mientras 
que para valores grandes de $r_{0}$ el espectro es prácticamente degenerado. Este comportamiento se hace más evidente en la figura 2.1; en el extremo izquierdo se observan los niveles de energía de la caja esférica impenetrable y en el derecho el espectro de energías del OAIL. Las energías del oscilador armónico isotrópico confinado OAIC se encuentran entre estos dos extremos [38].

Tabla 2. Eigenvalores de la energía $\epsilon_{n l}\left(r_{0}\right)$ del oscilador armónico isotrópico confinado en tres dimensiones para diferentes valores de $n$ y $l$ como función del radio de confinamiento $r_{0}$.

\begin{tabular}{|c|c|c|c|}
\hline$r_{0}$ & $\epsilon_{00}\left(r_{0}\right)$ & $\epsilon_{01}\left(r_{0}\right)$ & $\epsilon_{02}\left(r_{0}\right)$ \\
\hline 0.5 & 19.7745341792 & 40.4282764970 & 66.4897565349 \\
\hline 1.0 & 5.0755820152 & 10.2822569390 & $\begin{array}{llll}16.827 & 777 & 1098\end{array}$ \\
\hline 1.5 & 2.5049761785 & 4.9035904194 & 7.8717304877 \\
\hline 2.0 & 1.7648164387 & 3.2469470987 & 5.0100408656 \\
\hline 2.5 & 1.5514216545 & $2.688 \quad 1439638$ & 3.9535289034 \\
\hline 3.0 & 1.5060815272 & 2.5312924666 & 3.5982476989 \\
\hline 3.5 & 1.5003995211 & 2.5029101642 & 3.5125803181 \\
\hline 4.0 & 1.5000146030 & $2.500143778 \quad 1$ & 3.5008420738 \\
\hline 4.5 & 1.5000003041 & 2.5000038701 & 3.5000294123 \\
\hline 5.0 & 1.5000000036 & 2.5000000584 & 3.5000005567 \\
\hline 5.5 & 1.5000000002 & 2.5000000005 & 3.5000000058 \\
\hline 6.0 & 1.5000000000 & 2.5000000000 & 3.5000000003 \\
\hline$r_{0}=\infty^{*}$ & 1.5000000000 & 2.5000000000 & 3.500000000 \\
\hline$r_{0}$ & $\epsilon_{10}\left(r_{0}\right)$ & $\epsilon_{11}\left(r_{0}\right)$ & $\epsilon_{20}$ \\
\hline 0.5 & 78.9969211507 & 119.4024452520 & 177.6938438082 \\
\hline 1.0 & 19.8896965018 & 30.0134875924 & 44.5771712285 \\
\hline 1.5 & 9.1354220876 & 13.6537408930 & 20.1090029728 \\
\hline 2.0 & 5.5846390790 & 8.1595288816 & $\begin{array}{lll}11.764982 & 1223\end{array}$ \\
\hline 2.5 & 4.1842613183 & $\begin{array}{lllll}5.876 & 767 & 736 & 0\end{array}$ & 8.1534369588 \\
\hline 3.0 & 3.6642196450 & 4.9138976907 & 6.4733366162 \\
\hline 3.5 & 3.5233023363 & 4.5807989270 & $\begin{array}{llll}5.758 & 692 & 163 & 5\end{array}$ \\
\hline 4.0 & 3.5016915385 & 4.5083304308 & 5.5394217970 \\
\hline 4.5 & 3.5000623121 & 4.5004174712 & 5.5028722559 \\
\hline 5.0 & 3.5000012214 & 4.5000105730 & 5.5000987178 \\
\hline 5.5 & 3.5000000132 & $\begin{array}{lll}4.500 & 000 & 1425\end{array}$ & 5.5000017124 \\
\hline 6.0 & & 4.5000000008 & 5.5000000134 \\
\hline & & & \\
\hline
\end{tabular}

\footnotetext{
* Estos valores corresponden al OAIL en tres dimensiones calculados mediante fórmulas analíticas [100].
} 


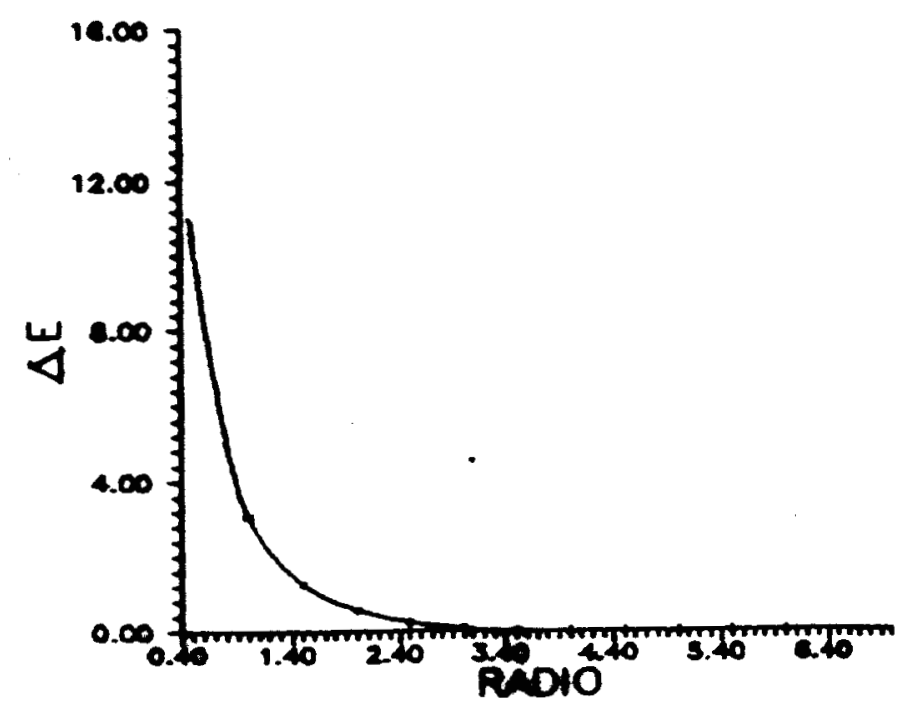

Figura 2.2: La separación de los niveles de energía $\Delta E\left(=E_{10}-E_{02}\right)$ se ha graficado como función de $r_{0}$. Para valores pequeños de $r_{0}$ no hay degeneración entre estos dos niveles, pero a medida que $r_{0}$ aumenta los niveles se aproximan a la degeneración.

Un hecho poco usual ocurre con los niveles $1 h$ y $3 s$ cuando $r_{0}$ disminuye. Existe cierto valor $r_{0} *$ para el cual estos niveles se cruzan y para valores menores de $r_{0} *$ el orden de estos niveles se invierte. Los estados $3 p$ y $1 i$ muestran un comportamiento análogo. Hay que observar que tanto el orden de los niveles como la separación entre ellos es función del radio de confinamiento $r_{0}$.

Una estimación de la no degeneración de la energía está dada, por ejemplo, por $\Delta E=E_{10}-E_{02}$. En la figura 2.2 mostramos una gráfica de $\Delta E$ como función de $r_{0}$. Nótese que la degeneración se rompe y crece el efecto de la separación en $\Delta E$ para valores de $r_{0} \leq 2.5 a$.u.

El hecho de que el espectro de energías no sea degenerado para valores pequeños de $r_{0}$ se puede entender de la siguiente manera: Para valores pequeños de $r_{0}$ podemos pensar que el problema es el de una partícula en una caja de paredes infinitas (cuyo espectro es no degenerado) más una perturbación de oscilador 
armónico $r_{0}^{2}$, así que el problema completo es no degenerado. Mientras que para valores grandes de $r_{0}$ el problema puede verse como el de un oscilador armónico perturbado por una caja de paredes impenetrables, así que el espectro será muy cercano a uno con degeneración, como era de esperarse.

Tabla 3. Valores esperados de $\left\langle r^{-2}\right\rangle,\left\langle r^{2}\right\rangle \mathrm{y}\left\langle r^{4}\right\rangle$ como función del radio de la caja $\left(r_{0}\right)$.

\begin{tabular}{|c|c|c|c|}
\hline$r_{0}$ & $\left\langle r^{-2}\right\rangle$ & $\left\langle r^{2}\right\rangle$ & $\left\langle r^{4}\right\rangle$ \\
\hline & $n=0, l=0$ & & \\
0.5 & 35.6703626921 & $7.0633325319 \mathrm{E}-2$ & $7.1240518727 \mathrm{E}-3$ \\
1.0 & 9.0241006941 & 0.2804491919 & 0.1125979005 \\
2.0 & 2.6958517746 & 0.9969257335 & 1.4783049734 \\
3.0 & 2.0244173259 & 1.4618641057 & 3.4785513901 \\
4.0 & 2.0000776828 & 1.4998046846 & 3.7479140456 \\
5.0 & 2.0000000216 & 1.4999999191 & 3.7499987701 \\
5.5 & 2.0000000007 & 1.4999999996 & 3.7499999994 \\
$r_{0}=\infty *$ & 2.0000000000 & 1.5000000000 & 3.75000000000 \\
& $n=0, l=1$ & & \\
0.5 & 15.6045790517 & $9.3625935728 \mathrm{E}-2$ & $1.0871505434 \mathrm{E}-2$ \\
1.0 & 3.9247112835 & 0.3729644642 & 0.1727284383 \\
2.0 & 1.0801733970 & 1.3944839053 & 2.4617968195 \\
3.0 & 0.6947107566 & 2.3374249913 & 7.3776557684 \\
4.0 & 0.6668569583 & 2.4982360443 & 8.7286620820 \\
5.0 & 0.6666667627 & 2.4999987516 & 8.7499792031 \\
5.5 & 0.6666666675 & 2.4999999866 & 8.7499997431 \\
$r_{0}=\infty *$ & 0.6666666667 & 2.5000000000 & 8.7500000000 \\
& $n=1, l=0$ & & \\
0.5 & 75.0099270868 & $8.0176719300 \mathrm{E}-2$ & $1.0977905107 \mathrm{E}-2$ \\
1.0 & 18.7738038158 & 0.3212800381 & 0.1758546450 \\
2.0 & 4.7740234899 & 1.3136755498 & 2.8376786605 \\
3.0 & 2.3631605071 & 2.8484973047 & 12.3605291432 \\
4.0 & 2.0061556647 & 3.4816007196 & 18.5015111189 \\
5.0 & 2.0000058010 & 3.4999753635 & 18.74955538 \\
6.0 & 2.0000000 & 3.500000000 & 18.75000000 \\
$r_{0}=\infty *$ & 2.0000000 & 3.500000000 & 18.75000000 \\
\hline
\end{tabular}

* Estos valores corresponden al OAIL en tres dimensiones calculados mediante fórmulas analíticas [100]. 


\subsection{Valores esperados de la posición $\left\langle r^{n}\right\rangle$}

Las funciones de onda obtenidas por este método no están normalizadas. Sin embargo, este hecho no es importante para calcular los valores esperados de la posición, porque hay otro procedimiento basado en el teorema de HellmannFeynman [78-80] mediante el cual estas cantidades se pueden calcular fácilmente sin el uso explícito de las funciones de onda. En esta sección, sin embargo, haremos una integración numérica de las funciones de onda obtenidas en la sección anterior para calcular el valor esperado de $r^{k}$, donde

$$
<r^{k}>=\frac{\int_{0}^{r_{0}} R^{2} r^{k} r^{2} d r}{\int_{0}^{r_{0}} R^{2} r^{2} d r}, \text { con } \mathrm{k}=\text { un número entero. }
$$

La integración fue realizada con un método adaptivo para la regla de Simpson, con este procedimiento numérico se obtuvieron los valores de $\left\langle r^{-2}\right\rangle,\left\langle r^{2}\right\rangle$, $\left\langle r^{-1}\right\rangle \mathrm{y}\left\langle r^{4}\right\rangle$ con 7 díigitos de precisión; los resultados de estos cálculos se encuentran en la Tabla 3. El comportamiento de los valores esperados es muy similar al de los eigenvalores de la energía. Estos varían monótonamente y se aproximan a los valores del OAIL cuando se incrementa el radio de confinamiento $r_{0}$.

Como lo indicamos antes, podemos calcular las eigenfunciones y eigenvalores de la energía a través de las ecuaciones (6) y (8), dando las condiciones iniciales de las funciones y sus derivadas en el origen y pidiendo que se satisfagan las condiciones de frontera en $r=r_{0}$.

Es importante mencionar que los nodos de las eigenfunciones quedan automáticamente determinados por estas dos condiciones también. No es necesario hacer ningún tipo de cambio en la forma de la función (6) (como en otros métodos [98-99]), para calcular los estados excitados. La posición de los nodos depende del radio $r_{0}$ de la caja impenetrable. Los nodos del OAIC se aproximan a la posición de los nodos del OAIL cuando $r_{0}$ aumenta, y son los mismos cuando $r_{0} \rightarrow \infty$. En la práctica podemos considerar que son iguales cuando $r_{0} \geq 6$. En la figura 2.3 mostramos la función de onda radial para 6 diferentes estados como función del 

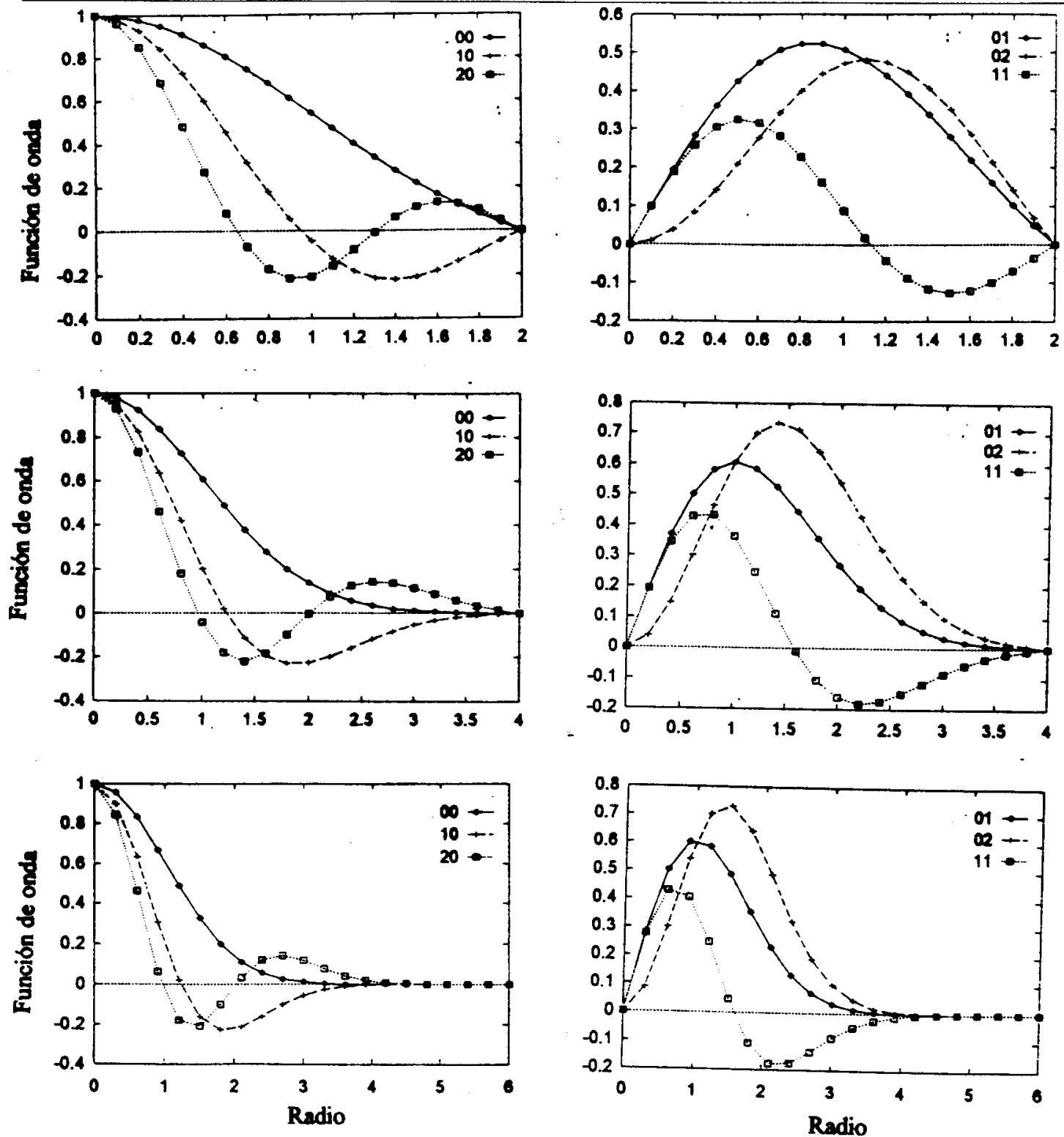

Figura 2.3: Gráficas de las funciones de onda radial para el estado base y los primeros 5 estados excitados, como función de $r_{0}$. Las funciones de onda no están normalizadas a la unidad. Para $r_{0}$ pequeño las funciones estan comprimidas dentro de la caja, pero a medida que $r_{0}$ aumenta las funciones se van pareciendo más a las del problema libre. 


\subsection{Conclusiones}

La principal ventaja del presente método es la posibilidad de poder calcular simultáneamente las eigenfunciones y los eigenvalores del oscilador armónico con una gran precisión (10 dígitos en los valores de la energía) y a un costo computacional muy bajo, ya que los cálculos aquí mostrados se pueden realizar en cualquier PC, desde una XT hasta una Pentium o Workstation.

Acerca de las funciones de onda podemos decir, que el efecto total del confinamiento mediante paredes impenetrables es el de comprimir a la función de onda dentro de la caja que la confina, pero la forma de la función confinada es semejante a la de la función libre, ya que el confinamiento del oscilador armónico rompe la degeneración de la energía pero no cambia la simetría del problema, de esta manera el número de nodos es el mismo que el del caso libre.

En trabajos anteriores se había calculado sólo los eigenvalores de la energía, pero en ninguno de ellos se reportaron los valores esperados de la posición. Estos valores pueden ser de mucha utilidad, ya que por ejemplo, el valor $\left\langle r^{2}\right\rangle$ esta relacionado con la polarizabilidad del sistema cuántico.

Como ya lo mencionamos en la introducción general de este trabajo, el modelo del oscilador armónico (unidimensional) confinado ha tenido su aplicación en problemas de Astrofísica, como por ejemplo, el estudio de la transformación protóndeuterón como fuente de energía en estrellas densas [33] y la relación fundamental masa-radio en la teoría de las enanas blancas [35].

El modelo del OAIC puede ser útil en el estudio del calor específico de sólidos bajo altas presiones empleando el modelo de Einstein. en este modelo los osciladores armónicos se encuentran desacoplados, desde luego, en este caso se tendría que resolver el problema unidimensional correspondiente. Sin embargo, el corrimiento observado en los niveles de energía en el caso tridimensional, debido a los efectos del confinamiento, indica un comportamiento similar, el cual tendría consecuencias en la función de partición correspondiente y por consiguiente en la variación del calor específico 
El estudio del OAIC es importante por sí mismo, pero es más interesante si pensamos en las posibles aplicaciones que de él se pueden hacer a sistemas más complejos. Como por ejemplo, el átomo de hidrógeno, el de helio y el ión molecular de hidrógeno confinados. En el siguiente capítulo haremos el cálculo variacional de las energías del átomo de hidrógeno confinado en la base del OIAC y las compararemos con el cálculo exacto que se obtiene al usar el método propuesto en este trabajo. 


\section{Capítulo 3}

\section{Átomo de hidrógeno 3D confinado}

\subsection{Introducción}

Los modelos más discutidos en la literatura, son sin lugar a dudas, el átomo de hidrógeno [1-29] y el oscilador armónico [30-50] confinados. Pese a la sencillez de estos sistemas, no son triviales y como ya mencionamos antes, no se conocen soluciones analíticas de estos problemas.

El modelo del átomo de hidrógeno confinado fue propuesto para explicar la estructura hiperfina del hidrógeno atómico atrapado en el cuarzo $\alpha$ [140], en la década de los 70s, Ley-Koo y Rubinstein [17] obtuvieron una solución exacta para el problema, sin embargo, para obtener los eigenvalores de la energía es necesario resolver numéricamente una ecuación complicada. Otro enfoque radicalmente diferente fue propuesto por Ludeña [13], al resolver el problema, usando el método de Hartree-Fock, empleando funciones tipo hidrogenoide multiplicadas por una función de corte, para satisfacer las condiciones de frontera. Una manera muy simple de atacar el problema de manera variacional fue propuesta por Marin y Cruz [24-25], en la cual las funciones de prueba se construyen al multiplicar la solución del problema libre por una función de corte que satisfaga las condiciones 
de frontera, como se indicó en el capítulo 1. Por otro lado Fernández y Castro [22, 31] usan una combinación del teorema hipervirial y teoría de perturbaciones para resolver el mismo problema. De estos métodos que hemos mencionado, sólo el de Ley-Koo y Rubinstein proporciona valores exactos de la energía. Otra manera de obtener valores exactos fue propuesta por Aquino[29]. El método fue presentado en' el capítulo 2 , los resultados de estos cálculos se presentan en este capítulo.

\subsection{Confinamiento dentro de cajas impenetra- bles}

\subsubsection{Hidrógeno estudiado en la base del OAIC}

Por muchos años la base del OAIL se ha usado para resolver problemas más complicados [101] , como el átomo de hidrógeno, la molécula de hidrógeno, el átomo de helio, etc. Una pregunta interesante surge de manera natural, ¿ Será la base del OAIC tan buena para problemas confinados como la base del OAIL para problemas libres?, daremos un respuesta mediante el cálculo de los eigenvalores de átomo de hidrógeno confinado.

Hace algunos años Moshinsky [101] calculó la energía del estado base dèl átomo de hidrógeno libre usando como base la del oscilador armónico isotrópico libre (OAIL). En esta sección mostraremos que usando esta misma idea, es posible hacer un cálculo similar para el átomo de hidrógeno confinado (por una caja esférica de paredes impenetrables) usando como base la del OAIC [38] obtenida en el capítulo anterior.

En la teoría cuántica no-relativista el hamiltoniano del átomo de hidrógeno confinado se puede escribir como:

$$
H^{\prime}=\frac{p^{2}}{2 m}+U\left(r^{\prime}\right)
$$

donde 


$$
U\left(r^{\prime}\right)=\left\{\begin{array}{cc}
-e^{2} / r^{\prime}, & \text { if } r \leq r_{0} \\
+\infty & \text { if } r>r_{0}
\end{array}\right.
$$

$r_{0}$ es el radio de la caja esférica de paredes impenetrables. Es conveniente transformar la ecuación (1) sumando y restando el término de oscilador armónico con frecuencia $w$ para $r<r_{0}$ :

$$
H^{\prime}=\frac{1}{2 m} p^{\prime 2}+\frac{1}{2} m w^{2} r^{2}-e^{2} / r^{\prime}-\frac{1}{2} m w^{2} r^{2}
$$

Haciendo el cambio de variable $r^{\prime}=a r$, donde $a=\sqrt{\frac{\hbar}{m w}}$, y la energía equivalente $\hbar w=\frac{m e^{4}}{\hbar^{2}}$, el hamiltoniano Ec. (3) es ahora

$$
H^{\prime}=\hbar w H=\frac{m e^{4}}{\hbar^{2}} H
$$

donde

$$
H=H_{b h o}-\frac{1}{r}-\frac{1}{2} r^{2}
$$

El término $H_{b h o}$ es el hamiltoniano del OAIC. Los eigenestados $R$ de la ec. (5) tienen una $l, m$ definidas,así que podemos desarrollar a $R$ en términos de los estados $\psi_{n l m}$ del oscilador confinado (OAIC):

$$
R=\sum_{n} \mid n l m>
$$

Para obtener los eigenvalores (de la energía) de (5) es suficiente con diagonalizar la matriz $\left\|<n^{\prime} l m|H| n l m>\right\|$ para $l, m$ fijas. Los elementos de matriz necesarios son

$$
<n^{\prime} l m|H| n l m>=E_{b h_{0}} \delta_{n^{\prime} n}-\frac{1}{2}<n^{\prime} l m\left|r^{2}\right| n l m>-<n^{\prime} l m|1 / r| n l m>
$$


Tabla 1. Eigenvalores de la energía $E_{n l}$ (en unidades atómicas) para el átomo de hidrógeno confinado como función del número de quanta $N$, para $r_{0}=2,4,5$ Bohrs.

\begin{tabular}{|c|c|c|c|c|c|c|}
\hline$N$ & $E_{00}$ & $E_{10}$ & $E_{20}$ & $E_{30}$ & $E_{40}$ & $E_{50}$ \\
\hline 0 & -0.0611 & & $r_{0}=2$ & & & \\
2 & -0.0839 & 3.4057 & & & & \\
4 & -0.1087 & 3.3776 & 9.4056 & & & \\
6 & -0.1159 & 3.3486 & 9.3708 & 17.9153 & & \\
8 & -0.1199 & 3.3400 & 9.3398 & 17.8766 & 28.9173 & \\
10 & -0.1217 & 3.3349 & 9.3300 & 17.8450 & 28.8763 & 42.4034 \\
12 & -0.1228 & 3.3325 & 9.3240 & 17.8345 & 28.8448 & 42.3612 \\
Exacto & -0.1250 & 3.3275 & 9.3142 & 17.8161 & 28.8135 & 42.2967 \\
& & & $r_{0}=4$ & & & \\
0 & -0.3783 & & & & & \\
2 & -0.3970 & 0.8374 & & & & \\
4 & -0.4642 & 0.6220 & 2.3658 & & & \\
6 & -0.4642 & 0.4455 & 2.0791 & 4.3978 & & \\
8 & -0.4732 & 0.4404 & 1.8981 & 4.1417 & 7.0545 & \\
10 & -0.4751 & 0.4277 & 1.8957 & 3.9913 & 6.8500 & 10.3535 \\
12 & -0.4776 & 0.4270 & 1.8823 & 3.9908 & 6.7159 & 10.1843 \\
Exacto & -0.4833 & 0.4202 & 1.8727 & 3.9665 & 6.6907 & 10.0406 \\
0 & & & $r_{0}=5$ & & & \\
2 & -0.3784 & & & & & \\
4 & -0.3974 & 0.8287 & & & & \\
6 & -0.4694 & 0.5778 & 2.2370 & & & \\
8 & -0.4857 & 0.2957 & 1.7409 & 3.7837 & & \\
10 & -0.4858 & 0.1579 & 1.2669 & 3.1064 & 5.4940 & \\
12 & -0.4895 & 0.1523 & 1.0708 & 2.5655 & 4.7643 & 7.5027 \\
Exacto & -0.4964 & 0.1413 & 1.0532 & 2.3823 & 4.2573 & 6.8101 \\
\end{tabular}

El valor exacto fue calculado mediante el procedimiento de la referencia [29].

El término $E_{b h o}$ representa el eigenvalor de la energía del OAIC, algunos de estos valores se encuentran en la Tabla 1, y el cálculo de los valores esperados de la posición se explicó en la sección anterior.

Por el momento sólo nos interesamos por los estados esféricamente simétricos, es decir, 1s, 2s, 3s, etc. Por esta razón podemos hacer $l=m=0$, así que sólo necesitamos calcular los elementos de matriz $\left\|<n^{\prime} 00|H| n 00>\right\|$. Para $N$ quanta necesitamos diagonalizar una matriz de $\left(\frac{1}{2} N+1\right)\left(\frac{1}{2} N+1\right)$, con $n=0,1, \ldots, \frac{1}{2} N$. Los resultados de nuestros cálculos para $r_{0}=2,4,5$ bohrs, se muestran en la Tabla 1, para el estado base y algunos estados excitados. 
De la Tabla 1 podemos observar una buena convergencia del estado base para 12 quanta; los estados excitados son los que convergen primero para valores pequeños de $r_{0}$. Sin embargo, para $r_{0} \geq 5$ el estado base converge antes que los estados excitados como en el caso del átomo de hidrógeno libre, de donde concluimos que la base del OIAC es útil para atacar éste problema. También es posible resolver el problema del oscilador armónico confinado en geometrías más complicadas, para después con esta base resolver el átomo de hidrógeno confinado en esa misma geometría.

\subsubsection{Cálculo de energias propias con alta precisión}

A continuación aplicaremos el método de series presentado en el capítulo anterior al problema del átomo de hidrógeno confinado dentro de paredes impenetrables.

La ecuación de Schrödinger independiente del tiempo (en unidades atómicas) de este problema está dada por:

$$
H=-\frac{1}{2} \nabla^{2}-1 / r+V(r)
$$

donde

$$
V(r)=\left\{\begin{array}{cc}
+\infty, & r \geq r_{0} \\
0, & r<r_{0}
\end{array}\right.
$$

$r_{0}$ es el radio de la caja esférica en bohrs, mientras que la energía esta en hartrees.

Ya que el problema es separable, solo resolveremos la ecuación radial, la cual se puede escribir de la siguiente manera:

$$
r^{2} \psi^{\prime \prime}=-2 r \psi^{\prime}+l(l+1) \psi-2 r \psi-2 \epsilon r^{2} \psi
$$

donde $l$ es el momento angular de la partícula. En esta ecuación hemos usado el símbolo ' para indicar derivada respecto a la posición. 
A continuación se hace un desarrollo en serie de Taylor para la función de onda $\psi$, alrededor de $r=0$, donde se conoce el valor inicial de la función.

$$
\psi(r)=\sum_{k} T_{k}
$$

donde

$$
T_{k}=\left(\psi^{(k)}(0) / k !\right) r^{k}
$$

Los coeficientes $T_{k}$ se pueden calcular de manera recursiva mediante

$$
T_{p+1}=-2 r\left[\epsilon r T_{p-1}+T_{p}\right] /[(p+1)(p+2)-l(l+1)]
$$

La función de onda puede evaluarse directamente usando (9a). Esta ecuación es válida para cualquier valor de $r$ que esté en el intervalo $\left[0, r_{0}\right]$. De la ecuación (4a) también se obtiene una expresión para evaluar las derivadas de la función de onda respecto a la energía:

$$
\partial \psi / \partial \epsilon=\sum_{m} \partial T_{m} / \partial \epsilon=\sum_{p} \dot{T}_{p}
$$

donde $\dot{T}_{p}$ denota la derivada de $T_{p}$ respecto a la energía. De la ecuación (4c), tenemos que

$$
\dot{T}_{p+1}=-2 r\left[\epsilon r \dot{T}_{p-1}-r T_{p-1}+\dot{T}_{p}\right] /[(p+1)(p+2)-l(l+1)]
$$

De las relaciones (11a), (12a) y la ec. (32) del capítulo 1, podemos construir cualquier eigenfunción, usando las condiciones iniciales: para $l=0$ (estados $s$ ), es suficiente con dar $\psi_{n}(0)=1$, y el valor de la derivada $\psi_{n}^{\prime}(0)=-1$, se obtiene de (4c). Para $l=1\left(\operatorname{los}\right.$ estados $p$ ), damos $\psi_{n}(0)=0$ y $\psi_{n}^{\prime}(0)=1$. Mientras que para $l=2(\operatorname{estados} d)$, debemos dar $\psi_{n}(0)=0, \psi_{n}^{\prime}(0)=0, \psi_{n}^{\prime \prime}(0)=1$. 
Una vez que se han obtenido los eigenvalores de la energía $\epsilon_{n}$ podemos calcular los coeficientes del desarrollo de la función de onda sustituyendo los valores de $\epsilon_{n}$ en la ecuación (4c) y evaluando $T_{p}$ en $r=1$. Así se obtiene la siguiente relación para la función de onda:

$$
\psi_{n}(r)=\sum_{m} T_{m}\left(r=1, \epsilon_{n}\right) r^{m} .
$$

Tabla 2. Eigenvalores de la energía $\epsilon\left(r_{0}\right)$ para el estado base y los dos primeros estados excitados $(l=0)$ del átomo de hidrógeno confinado como función del radio de la caja $r_{0}$. Las energías están en hartrees y las distancias en Bohrs.

\begin{tabular}{|c|c|c|c|}
\hline$r_{0}$ & $1 s\left(\epsilon_{1}\right)$ & $2 s\left(\epsilon_{2}\right)$ & $3 s,\left(\epsilon_{3}\right)$ \\
\hline 1.05486 & 1.9999831673 & 14.7326547665 & 36.5466762735 \\
2.00000 & -0.12500000000 & 3.32750915649 & 9.31415043551 \\
4.08671 & -0.48533085511 & 0.38576150184 & 1.77514809767 \\
7.00000 & -0.49986257755 & -0.05126039361 & 0.39224114311 \\
8.00000 & -0.49997510044 & -0.08473872135 & 0.24649197699 \\
10.00000 & -0.49999926328 & -0.11280621029 & 0.09142232240 \\
12.00000 & -0.49999998015 & -0.12141586728 & 0.01975295004 \\
14.00000 & -0.49999999949 & -0.12401502943 & -0.01606557644 \\
16.00000 & -0.49999999987 & -0.12475028444 & -0.03472990828 \\
19.00000 & -0.50000000000 & -0.12497231269 & -0.04769156796 \\
21.00000 & -0.50000000000 & -0.1249940820 & -0.05153736974 \\
23.00000 & -0.50000000000 & -0.12499879429 & -0.05355614753 \\
25.00000 & -0.50000000000 & -0.12499976370 & -0.05459245098 \\
30.00000 & -0.50000000000 & -0.12499999646 & -0.05542367049 \\
35.00000 & -0.50000000000 & -0.12499999995 & -0.05554112309 \\
42.00000 & -0.50000000000 & -0.1250000000 & -0.0555550675 \\
48.00000 & -0.50000000000 & -0.12500000000 & -0.055555553 \\
$(r=\infty)^{a}$ & -0.5000000000 & -0.1250000000 & -0.0555555556 \\
\hline
\end{tabular}

${ }^{a}$ Este valor fue calculado mediante las fórmulas analíticas para el átomo libre.

El cálculo de los eigenvalores de la energía y de las eigenfunciones es muy rápido y preciso. En la Tabla 2 se muestran algunos de los valores de la energía obtenidos mediante este método; todos los valores tienen una precisión de 10 cifras.

Los eigenvalores de la energía se aproximan a los del átomo de hidrógeno libre cuando $r_{0}$ aumenta, como puede observarse de la Tabla 2. Debe notarse que este comportamiento es monótono decreciente. 
En la tabla 3 comparamos los resultados obtenidos mediante este método [29] con los que se obtienen usando el método variacional de Marín y Cruz [24-26], el método de Fernández y Castro [22], el de Ludeña [13] y los valores obtenidos por Ley-Koo y Rubinstein [17]. Es evidente que los resultados obtenidos por el método propuesto en este trabajo son mucho más precisos.

Tabla 3. Eigenvalores de la energía $\epsilon\left(r_{0}\right)$ para el estado base del átomo de hidrógeno confinado como función del radio de la caja $r_{0}$. Las energías están en hartrees y las distancias en Bohrs.

\begin{tabular}{|c|c|c|c|c|c|}
\hline$r_{0}$ & {$[13]$} & {$[24-25]$} & {$[22]$} & {$[17]$} & Actual \\
\hline 0.53622 & & 12.6237 & & 12.5000 & 12.50019917931 \\
0.81000 & 4.3920 & 4.4275 & 4.3960 & & 4.39164157090 \\
1.15000 & 1.4860 & 1.4953 & 1.4871 & & 1.48625280550 \\
1.22195 & & 1.19015 & & 1.18345 & 1.18344646955 \\
1.71100 & 0.1260 & 0.1267 & 1.1263 & & 0.12624365292 \\
2.00000 & -0.1250 & -0.1250 & & -0.1250 & -0.12500000000 \\
2.20050 & -0.2320 & -0.2321 & -0.2323 & & -0.23225436144 \\
2.44558 & & -0.3195 & & -0.3200 & -0.32000030292 \\
2.80700 & & -0.39665 & -0.3939 & & -0.39777647173 \\
3.04187 & & -0.4272 & & -0.42865 & -0.42866962486 \\
3.19200 & & -0.44155 & -0.4463 & & -0.44321857319 \\
3.52870 & -0.4660 & -0.46385 & & & -0.46586834749 \\
4.08671 & & -0.48315 & & -0.48535 & -0.48533085511 \\
4.41530 & -0.4910 & -0.48905 & & & -0.49112101703 \\
5.02000 & -0.4960 & -0.4948 & & & -0.49652777253 \\
5.80119 & & -0.4978 & & -0.4990 & -0.49900149259 \\
6.22530 & -0.4990 & -0.49855 & & & -0.49950038051 \\
\hline
\end{tabular}

En la tabla 4 reportamos los eigenvalores de la energía para los estados $2 p$ y $3 d$ obtenidos mediante este método [29] y los de otros autores que encontramos en la literatura. Los resultados que se obtienen con el presente método son mucho más precisos que los reportados con anterioridad, vemos nuevamente que cuando el tamaño de la caja aumenta estos eigenvalores se acercan a los del átomo de hidrógeno libre.

Debemos notar que cuando el radio $r_{0}$ de la caja esférica impenetrable es muy grande, el ordenamiento de los niveles de energía es prácticamente el mismo que el del átomo libre, pero a medida que $r_{0}$ decrece, se nota inmediatamente que la degeneración de los niveles de energía se rompe. Es decir, debido al confinamiento 


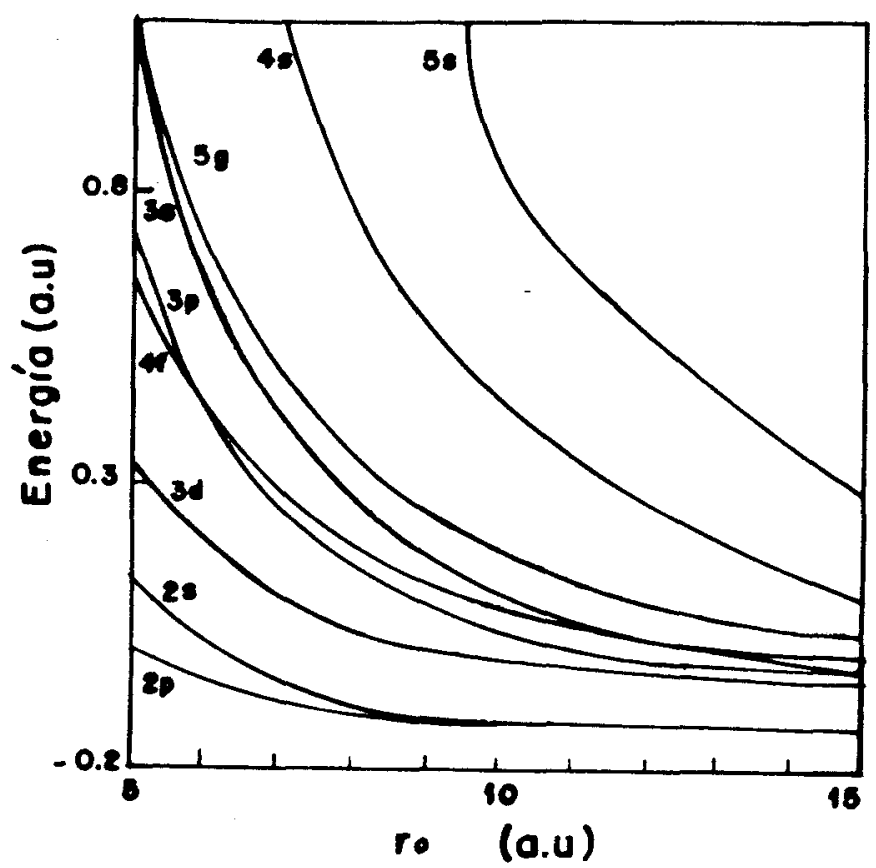

Figura 3.1: Al confinar al átomo de hidrógeno se rompe la degeneración accidental y se separan las energías orbitales de tal manera que $E_{2 p}<E_{2 s}, E_{3 d}<E_{3 p}<E_{3 s}$, etc.

espacial se rompe la degeneración accidental y las energias orbitales se separan, este comportamiento se hace más evidente en la figura 3.1. Para radios muy pequeños las energías deben ser más parecidas a las de una partícula en una caja, ya que la energía total es positiva. Lo cual indica que la energía cinética es mayor que la potencial y el electrón permanece cerca del núcleo gracias a la caja de paredes infinitas más que a la atracción electrostática.

La degeneración accidental se rompe y se observa que $E_{2 p}<E_{2 s}, E_{3 d}<E_{3 p}<$ $E_{3 s}$, etc. Este nuevo ordenamiento de los niveles de energía es de crucial importancia. Si los átomos multielectrónicos bajo confinamiento espacial siguen este mismo ordenamiento de los niveles de energía, entonces sus propiedades químicas pueden cambiar radicalmente, y esto es lo que se experimentalmente se ha encontrado. Por ejemplo, consideremos al sodio libre, cuya configuración electrónica es $1 s^{2} 2 s^{2} 2 p^{6} 3 s^{1}$, bajo altas presiones cambiaría a $1 s^{2} 2 p^{6} 2 s^{2} 3 d^{1}$ de acuerdo con la figura 3.1 , con lo cual ya no se comportaría como un metal alcalino. Esto es, suponiendo que el confinamiento se realiza mediante paredes impenetrables, pero 
un caso más realista sugiere que la paredes sean penetrables.

\subsubsection{Valores esperados de potencias de la coordenada radial}

La función de onda obtenida por este método no está normalizada, pero este hecho no es relevante para calcular los valores esperados de la posición, ya que se puede normalizar. Sin embargo, estas cantidades pueden calcularse aun sin conocer explícitamente la función de onda, mediante el teorema de Hellmann-Feynman $[78-80]$ :

$$
d E / d \alpha=<\partial H / \partial \alpha>
$$

donde $\alpha$ es cualquier parámetro en el hamiltoniano.

Entonces, si sumamos a nuestro hamiltoniano $\mathrm{H}$ el término $\alpha r^{n}$, con $n$ un entero y resolvemos el problema de eigenvalores de este nuevo hamiltoniano $H^{\prime}$,

$$
H^{\prime}=H+\alpha r^{n},
$$

el cálculo de $<r^{n}>$ se reduce a

$$
<r^{n}>=<\partial H^{\prime} / \partial \alpha>
$$

El procedimiento numérico para calcular $\left\langle r^{n}>\right.$ es el siguiente: Se calculan los eigenvalores de la energía para el nuevo hamiltoniano $H^{\prime}$, ecuación (8). para valores pequeños de $\alpha$, y entonces se obtiene la derivada numéricamente. Los resultados que se presentan en la tabla 5 se obtuvieron usando un algoritmo de 5 puntos para el cálculo numérico de la derivada [95-96]. De esta tabla podemos observar la buena convergencia de los valores esperados hacia los valores exactos del átomo de hidrógeno libre, a medida que el radio de la caja va aumentando. 
Tabla 4. Eigenvalores de la energía $\epsilon\left(r_{0}\right)$ para los estados excitados $2 p$ y $3 s$ del átomo de hidrógeno confinado como función del radio de la caja $r_{0}$. Las energías están en hartrees y las distancias en Bohrs.

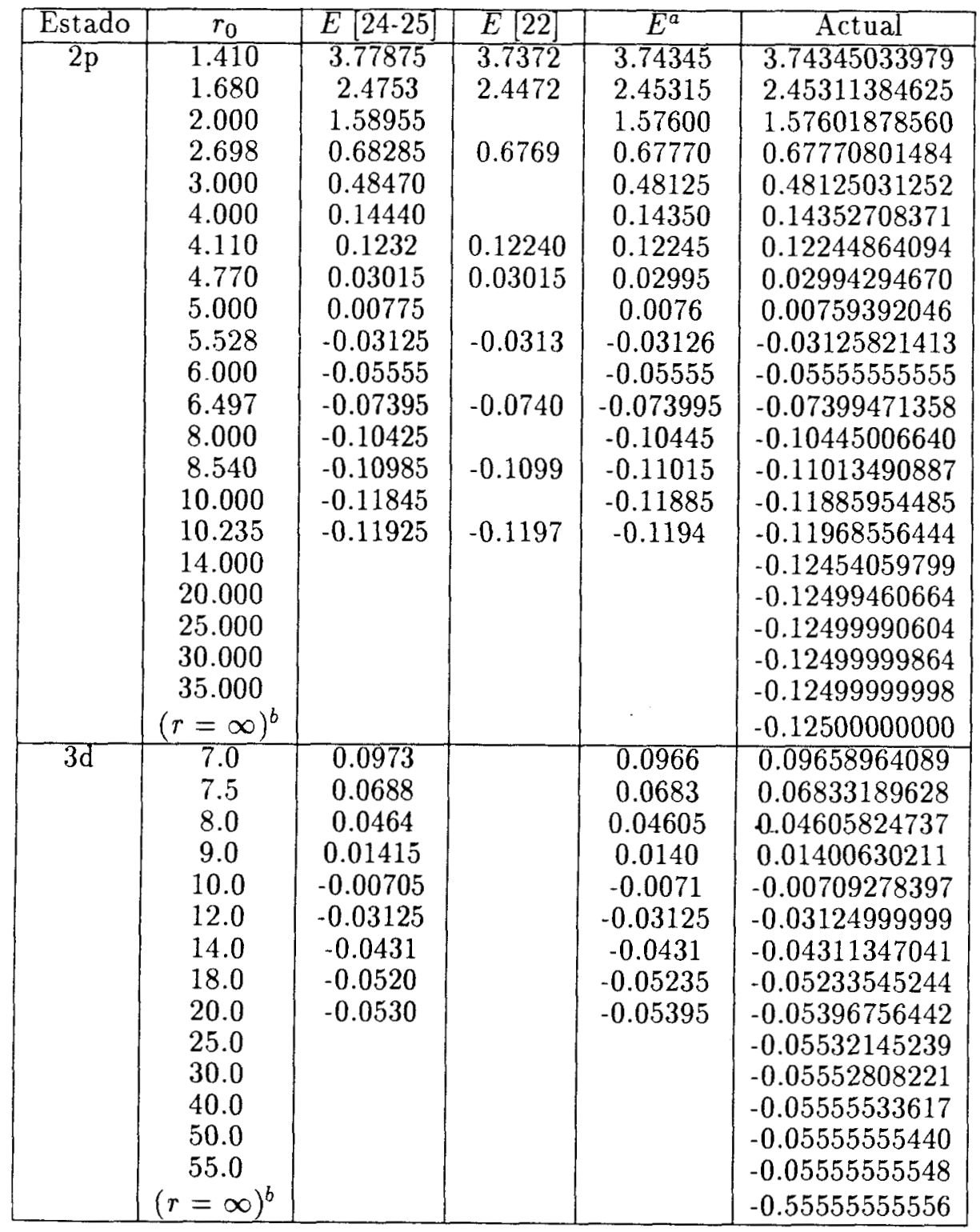

${ }^{a}$ Valores calculados por Marín y Cruz [24] usando el método de Ley-Koo [17].

${ }^{b}$ Calculado mediante las fórmulas analíticas [100]. 


\subsubsection{Propiedades físicas}

Con la información que hemos obtenido hasta el momento tenemos la posibilidad de determinar otras cantidades físicas importantes. Estamos interesados en las propiedades del estado base del átomo de hidrógeno confinado. Algunas de ellas son el desdoblamiento hiperfino [12], dado por el término de contacto de Fermi,

$$
A=(2 / 3) g \beta g_{n} \beta_{n} R_{\nu 0}^{2}(0)
$$

donde

$$
R_{\nu 0}^{2}(0)=1 /<\psi \mid \psi>
$$

Tabla 5. Valores esperados $\langle 1 / r\rangle$ de y $\left\langle r^{2}\right\rangle$ para el estado base y algunos estados excitados.

\begin{tabular}{|c|c|c|c|c|c|}
\hline$r_{0}$ & $\langle 1 / r\rangle$ & $\left\langle r^{2}\right\rangle$ & $r_{0}$ & $\langle 1 / r\rangle$ & $\left\langle r^{2}\right\rangle$ \\
\hline & $1 s$ & & & $2 s$ & \\
0.53662 & 4.78628287 & 0.07689770 & 2.00000 & 1.64627014 & $0.13320904 \mathrm{E}+1$ \\
1.05486 & 2.57504998 & 0.27969993 & 4.93358 & 0.63685783 & $0.86689450 \mathrm{E}+1$ \\
2.00000 & 1.53516170 & 0.87482539 & 7.00000 & 0.41884859 & $0.16775775 \mathrm{E}+2$ \\
3.04187 & 1.18789094 & 1.65527121 & 10.0000 & 0.29883743 & $0.28524320 \mathrm{E}+2$ \\
4.08671 & 1.06183597 & 2.31605115 & 15.0000 & 0.25415513 & $0.39737509 \mathrm{E}+2$ \\
5.80119 & 1.00738609 & 2.86000034 & 20.0000 & 0.25017311 & $0.4184913 \mathrm{E}+2$ \\
7.00000 & 1.00134706 & 2.96554751 & 30.0000 & 0.25000008 & $0.4199984 \mathrm{E}+2$ \\
8.00000 & 1.00029451 & 2.99063240 & 35.0000 & 0.25000001 & $0.4199998 \mathrm{E}+2$ \\
10.0000 & 1.00001169 & 2.99945950 & $(r=\infty)^{a}$ & 0.25000000 & $0.4200000 \mathrm{E}+2$ \\
12.0000 & 1.00000039 & 2.99997501 & & & \\
14.0000 & 1.00000001 & 2.99999900 & & & \\
16.0000 & 1.00000000 & 2.99999999 & & & \\
$(r=\infty)^{a}$ & 1.00000000 & 3.0000000 & & & \\
& $3 s$ & & & $2 p$ & \\
4.08671 & 0.89190756 & $0.5822915 \mathrm{E}+1$ & 2.00000 & 0.97234328 & $0.1405663 \mathrm{E}+1$ \\
10.0000 & 0.32310459 & $0.3816705 \mathrm{E}+2$ & 5.00000 & 0.42922962 & $0.7726190 \mathrm{E}+1$ \\
16.0000 & 0.17628677 & $0.9890471 \mathrm{E}+2$ & 10.0000 & 0.27537090 & $0.2178063 \mathrm{E}+2$ \\
30.0000 & 0.11251371 & $0.2000751 \mathrm{E}+3$ & 20.0000 & 0.25007388 & $0.2993300 \mathrm{E}+2$ \\
42.0000 & 0.11112039 & $0.2069195 \mathrm{E}+3$ & 30.0000 & 0.25000003 & $0.2999771 \mathrm{E}+2$ \\
48.0000 & 0.11111177 & $0.2069937 \mathrm{E}+3$ & 35.0000 & 0.25000000 & $0.2999998 \mathrm{E}+2$ \\
$(r=\infty)^{a}$ & 0.11111111 & $0.2070000 \mathrm{E}+3$ & $(r=\infty)^{a}$ & 0.25000000 & $0.3000000 \mathrm{E}+2$ \\
\hline
\end{tabular}

${ }^{a}$ Valores calculados mediante fórmulas analíticas 
El apantallamiento magnético nuclear [6], dado por la constante de apantallamiento diamagnético,

$$
\sigma=e^{2}<1 / r>\left(3 \mu c^{2}\right) .
$$

La polarizabilidad, dada por la aproximación de Kirkwood [1],

$$
\alpha=4<r^{2}>^{2} /\left(9 a_{0}\right)
$$

Tabla 6. Valores de la constante de desdoblamiento hiperfino $A$, la constante de apantallamiento magnético $\sigma$, la polarizabilidad $\alpha$ y la presión $P$, como función del radio $r_{0}$. Los valores en $r=\infty$ se calcularon mediante fórmulas analíticas [100].

\begin{tabular}{|c|c|c|c|c|}
\hline$A(m T)$ & & & $\sigma\left(e^{2} / 3 \mu a_{0} c^{2}\right)$ & \\
\hline$r_{0}$ & {$[17]$} & Actual & {$[17]$} & Actual \\
0.53622 & 2317.0307 & 2317.0715 & 4.7863 & 4.78628287 \\
1.05486 & 431.3955 & 431.39378 & 2.5751 & 2.57504998 \\
2.00000 & 120.5113 & 120.51127 & 1.5352 & 1.53516171 \\
3.04187 & 69.3406 & 69.34056 & 1.1789 & 1.18789094 \\
4.08671 & 56.0665 & 56.06651 & 1.0618 & 1.06183597 \\
5.37065 & 51.7762 & 51.77635 & 1.0131 & 1.01307033 \\
5.80199 & 51.3202 & 51.32174 & 1.0074 & 1.00738609 \\
8.00000 & & 50.78275 & & 1.00029451 \\
9.00000 & & 50.76632 & & 1.00006020 \\
10.0000 & & 50.76303 & & 1.00001169 \\
12.0000 & & 50.76228 & & 1.00000039 \\
\hline$(r=\infty)$ & & 50.7620 & & 1.00000000 \\
\hline$\alpha\left(10^{-24} c m^{3}\right)$ & & & $P\left(10^{6} a t m\right)$ & \\
$r_{0}$ & {$[17]$} & Actual & {$[17]$} & Actual \\
0.53622 & 0.0004 & 0.0003872 & 4463.2770 & 4463.937894 \\
1.05486 & 0.0052 & 0.0051238 & 129.4175 & 129.430937 \\
1.51301 & 0.0192 & 0.0191248 & 58.6808 & 58.688489 \\
2.00000 & 0.0504 & 0.0501250 & 3.7115 & 3.740753 \\
4.08671 & 0.3533 & 0.3513245 & 0.0309 & 0.030865 \\
5.37065 & 0.5092 & 0.5063356 & 0.0026 & 0.002537 \\
5.80119 & 0.5391 & 0.5357289 & 0.0011 & 0.001111 \\
10.0000 & & 0.5892480 & & $3.042 \mathrm{E}-7$ \\
14.0000 & & 0.5894600 & & $1.088 \mathrm{E}-10$ \\
16.0000 & & 0.5894603 & & $1.354 \mathrm{E}-12$ \\
17.0000 & & 0.5894604 & & $5.664 \mathrm{E}-13$ \\
\hline$(r=\infty)$ & & 0.5894604 & \multicolumn{3}{|c|}{0.00000} \\
\hline
\end{tabular}


y la presión,

$$
P=-\left(1 / 4 \pi r_{0}^{2}\right)\left(d E / d r_{0}\right)=(2 E-<V>) /\left(4 \pi r_{0}^{2}\right)
$$

donde $\langle V\rangle=-<1 / r>$.

Ya hemos visto como calcular los valores esperados de la posición sin el conocimiento explícito de la función de onda, pero para calcular el desdoblamiento hiperfino necesitamos conocer

$$
<\psi \mid \psi>=\int_{0}^{\tau_{0}} \psi^{2}(r, \epsilon) r^{2} d r
$$

Esta integral puede obtenerse numéricamente mediante la regla de Simpson extendida [95], los valores de las cantidades físicas antes mencionadas que se obtienen por este método los reportamos en la tabla 6 .

\subsubsection{Funciones de onda}

- Como ya se mencionó, mediante el presente método se pueden determinar simultáneamente los eigenvalores y las eigenfunciones del átomo de hidrógeno confinado por paredes impenetrables. El efecto de las paredes impenetrables sobre el espectro de energías es romper la degeneración que existía en el momento angular, pero la simetría del problema permanece inalterada. Así que la parte angular de la función de onda continúa estando representada por los armónicos esféricos, mientras que la parte radial mantiene intacto el número de nodos que se observan en el caso libre. El efecto del confinamiento lo podemos ver en la figura 3.1, en donde se muestran las funciones de onda sin normalizar para los estados $1 s, 2 s$ y $3 s$. Las funciones de onda se ven comprimidas por las paredes impenetrables, pareciéndose más a las funciones de la caja esférica para radios muy pequeños y recobrando su forma exponencial a medida que el radio de la caja se incrementa. 

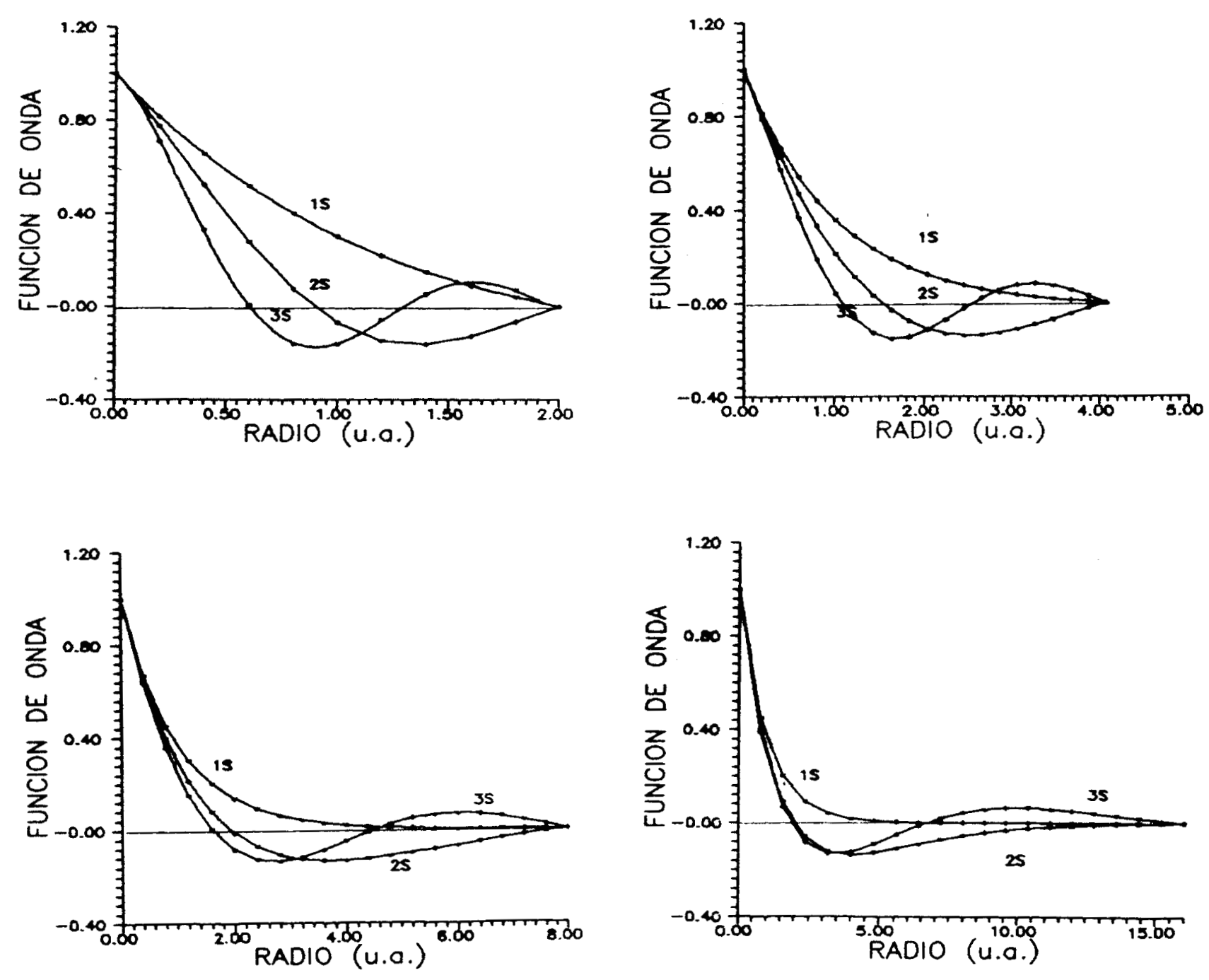

Figura 3.2: Gráfica de las funciones de onda $1 s, 2 s$ y $3 s$, como función del radio de la caja impenetrable. Para la gráfica (a) $r_{0}=2 a_{0}$, (b) $r_{0}=4.1 a_{0}$, (c) $r_{0}=8 a_{0}$ y (d) $r_{0}=16 a_{0}$. Las funciones de onda se comprimen al interior de la caja de radio $r_{0}$ como efecto del encajonamiento; en esta figura las funciones de onda no están normalizadas. 


\section{3 Átomo de $\mathrm{H}$ con condiciones a la frontera tipo Neumann}

La ecuación de Schrödinger independiente del tiempo (en unidades atómicas) está dada por la ecuación (1) donde ahora el potencial $U(r)=0$ para toda $r$. Las condiciones de frontera que deben satisfacer la funciones de onda radial son las condiciones de frontera de Neumann :

$$
\psi^{\prime}\left(r=r_{0}\right)=0
$$

donde el simbolo' significa derivada respecto a $r$. A continuación encontraremos los eigenvalores que corresponden a estados ligados únicamente.

Las soluciones de este problema son las dadas por las ecuaciones $4 a, 4 b$ y $4 c$, donde las incógnitas son precisamente los eigenvalores de la energía, que ahora se determinarán a partir de las condiciones de frontera de Neumann (23).

Para determinar los eigenvalores de la energía procedemos de manera análoga al tratamiento de la sección 3.2. Mediante el esquema de Newton-Raphson [95-97] podemos encontrar los eigenvalores de la energía dentro de una precisión que fijemos.

$$
\epsilon_{i+1}=\epsilon_{i}-\frac{\psi^{\prime}\left(r_{0} ; \epsilon_{i}\right)}{\dot{\psi}^{\prime}\left(r_{0} ; \epsilon_{i}\right)}
$$

donde $\dot{\psi}^{\prime}=\partial \psi^{\prime} / \partial \epsilon$. Esta última derivada se toma numéricamente, haciendo uso de la regla de diferencias centrales de seis puntos. En la tabla 7 se muestran los eigenvalores obtenidos mediante este procedimiento y los obtenidos por Killingbeck [118] y por Friedman et al [119] usando el método de elemento finito. 
Tabla 7. Eigenvalores de la energía para el átomo de hidrógeno para el estado base $(l=0)$ con condiciones de Neumann, para diferentes valores de $r_{0}$.

\begin{tabular}{|c|c|c|c|}
\hline$T=0$ & & & \\
\hline$r_{0}$ & Friedman & Killingbeck & Actual \\
\hline 0.5 & -3.055 & -3.05513386 & $\begin{array}{l}-3.055133860168\end{array}$ \\
\hline 1.0 & -1.561 & -1.56146218 & -1.561462181919 \\
\hline 2.0 & -0.829 & -0.829506651 & -0.829506651041 \\
\hline 3.0 & -0.609 & -0.609196267 & -0.609196267233 \\
\hline 4.0 & -0.529 & -0.529302461 & -0.529302460977 \\
\hline 5.0 & -0.504 & -0.506158374 & $-0.506 \quad 158373699$ \\
\hline 6.0 & -0.495 & -0.501139438 & -0.501139438570 \\
\hline 7.0 & & & -0.500200188926 \\
\hline 8.0 & & & -0.500034174169 \\
\hline
\end{tabular}

De esta tabla (7) podemos apreciar que los resultados obtenidos mediante el método del elemento finito pierden precisión a medida que se incrementa $r_{0}$, mientras que los resultados de Killingbeck aunque mantienen la precisión, son una aproximación a los resultados que aquí presentamos.

\subsection{El átomo de $\mathrm{H}$ dentro de una caja pene- trable}

Un modelo más físico para el átomo de hidrógeno confinado sugiere que las paredes de la caja que lo contienen, deberían de ser penetrables. El hamiltoniano asociado a la ecuación de Schrödinger para este problema es

$$
H=-\frac{1}{2} \nabla^{2}+V(r)
$$

donde ahora el potencial es

$$
V(r)=\left\{\begin{array}{cc}
V_{0}, & r \geq r_{0} \\
-1 / r, & r<r_{0}
\end{array}\right.
$$

$V_{0}$ es una constante no negativa. 
La ecuación de Schrödinger radial es

$$
\psi^{\prime \prime}+\frac{2}{r} \psi^{\prime}-\frac{l(l+1)}{r^{2}}-2 V(r) \psi+2 E \psi=0,
$$

La solución para la región $r<r_{0}$ está dada por las ecuaciones $11 a, 11 b$ y $11 c$ y la llamaremos $\psi^{i}$

$$
\psi^{i}=\sum_{0}^{\infty} T_{p}
$$

Para la región $r \geq r_{0}$ la ecuación (20) se reduce a

$$
\frac{d^{2} \chi}{d r^{2}}-\frac{l(l+1)}{r^{2}}-k^{2} \chi=0
$$

donde $k^{2}=2\left(V_{0}-E\right)$ y $\chi=r \psi^{e}$.

Para el caso $l=0$ la solución se obtiene inmediatamente

$$
\psi^{e}(r)=B e^{-k r} / r
$$

Para determinar los eigenvalores de la energía basta con exigir que las funciones $\psi^{i}(r)$ y $\psi^{e}(r)$, sean continuas y tengan derivada continuas en $r=r_{0}$, lo que nos lleva a encontrar las soluciones de la ecuación

$$
\frac{\psi^{i^{\prime}}\left(r_{0} ; E\right)}{\psi^{i}\left(r_{0} ; E\right)}=\frac{\psi^{e^{\prime}}\left(r_{0} ; E\right)}{\psi^{e}\left(r_{0} ; E\right)}=-\left(k+\frac{1}{r_{0}}\right)
$$

Para encontrar las raíces de la ecuación (24) hacemos uso nuevamente del método de Newton-Raphson [95-97]. En la Tabla 8 se reportan algunos de los eigenvalores de la energía obtenidos por este procedimiento para diferentes valores de $r_{0}$. 
Tabla 8. Eigenvalores de la energía del átomo de hidrógeno para el estado base $(l=0)$ confinado dentro de paredes penetrables. El alto de la barrera es $V_{0}=0.5$, para diferentes valores de $r_{0}$, las energía estan en hartrees y las distancias en bohrs.

\begin{tabular}{|c|c|c|}
\hline$r_{0}$ & Ref.[17] & Actual \\
0.73918 & 0.4132 & 0.4132259339 \\
0.83807 & 0.29585 & 0.2958597207 \\
1.03054 & 0.0800 & 0.0799966714 \\
1.25130 & -0.1033 & -0.103306791 \\
1.53583 & -0.2551 & -0.255103218 \\
1.72210 & -0.3200 & -0.319998880 \\
1.96281 & -0.37805 & -0.378071403 \\
2.46766 & -0.4450 & -0.444998290 \\
3.13886 & -0.4806 & -0.480584480 \\
3.57457 & -0.49015 & -0.490147954 \\
4.14964 & -0.4960 & -0.496023853 \\
4.57972 & -0.4980 & -0.498005999 \\
\hline
\end{tabular}

\subsection{Conclusiones}

Han sido muchos y muy variados los métodos que se han empleado en el estudio del átomo de hidrógeno confinado por paredes penetrables e impenetrables, y bajo condiciones de Neumann. El método que hemos propuesto ofrece amplias ventajas sobre los anteriores, pero tal vez la más importante es que produce resultados de muy alta precisión con muy poco esfuerzo y no requiere de grandes recursos computacionales.

Este modelo, como ya se mencionó en la introducción general, ha sido utilizado en el estudio de centros de color e impurezas hidrogenoides embebidas en materiales semiconductores [85-92], así como en el estudio del hidrógeno atómico atrapado en el cuarzo $\alpha[140]$.

Es muy interesante saber que en el átomo de hidrógeno confinado por paredes impenetrables se rompe la degeneración accidental y que los niveles de energía altos se cruzan e invierten su ordenamiento. Según el modelo de capas para átomos multielectrónicos, algo semejante debe de suceder con ellos, lo cual nos indica que a altas presiones los elementos químicos pueden tener propiedades diferentes a las del caso libre. Un enfoque más realista nos obliga a pensar en un 
confinamiento con paredes penetrables, lo que puede cambiar el ordenamiento de los niveles de momento angular alto.

En problemas de Astrofísica con este modelo se pueden preveer transiciones entre niveles de energía invertidos por el efecto del confinamiento, asociados a la posible existencia de hidrógeno atómico sometido a altas presiones.

Para tener una idea más completa del comportamiento de los átomos multielectrónicos bajo altas presiones, debemos recurrir a un modelo más elaborado, por ejemplo, Hartree-Fock o CI con confinamiento impenetrable, como lo hizo Ludeña para algunos átomos ligeros [57-58], aunque como ya lo mencionamos, el caso más realista sugiere que las fronteras sean penetrables. Este problema aún permanece abierto. 


\section{Capítulo 4}

\section{El átomo de hidrógeno 2D confinado}

\subsection{Introducción}

El estudio del átomo de hidrógeno (libre) en tres dimensiones es un tópico obligado en casi todos los libros de texto [108], mientras que los análisis en una dimensión [120-126] y dos dimensiones [126-135] por lo regular no se incluyen. Estos dos últimos problemas también ya han sido resueltos y sus soluciones se encuentran en la literatura [120-135]. En el átomo de hidrógeno libre (en una, dos o tres dimensiones) $[108,120-135]$ se pueden encontrar los eigenvalores de la energía en forma analítica, mientras que en el caso confinado esto no es posible.

El modelo del átomo de hidrógeno en dos dimensiones libre fue propuesto por Kohn y Luttinger [133] para describir el movimiento de un electrón en un cristal altamente anisotrópico. por lo tanto, las soluciones de este sistema tienen interés para el estudio de los efectos anisotrópicos en sólidos [137-139].

Existen ciertos fenómenos físicos en los que los sistemas cuánticos ya no pueden considerarse libres, sino que están confinados dentro de recintos que tienen fronteras de extensión finita. Un ejemplo de ello es el átomo de hidrógeno (tri- 
dimensional) atrapado dentro del cuarzo- $\alpha$ [140]. El primer modelo que se usó para explicar este fenómeno fue considerar al hidrógeno confinado dentro de una caja esférica impenetrable $[13,17,22-25,29,136]$, encontrándose que este modelo predecía cualitativamente las propiedades del sistema físico. En este caso ya no es posible encontrar en una forma analítica sencilla a los eigenvalores de la energía y debe de recurrirse a un método numérico para determinarlos. Sin embargo, para la solución de este tipo de problemas, se ha recurrido exitosamente a diversos métdos aproximados [31].

Antes de continuar debemos de definir al sistema cuántico que llamaremos átomo de hidrógeno en dos dimensiones: Si el movimiento del electrón alrededor del núcleo (puntual) cargado positivamente está completamente restringido a un plano, entonces el átomo de hidrógeno se considerará en dos dimensiones. Esto podría corresponder físicamente al caso de una impureza hidrogenoide ubicada en la interficie entre dos materiales semiconductores. En este trabajo estudiaremos al átomo de hidrógeno en dos dimensiones confinado mediante una pared circular impenetrable, empleando el método variacional directo $[25,49]$.

En el átomo de hidrógeno en dos dimensiones, el potencial de atracción electrostática es el potencial Coulombiano $V(r)=-e^{2} / r$ donde $e$ es valor de la carga eléctrica del electrón de masa $m$ y $r$ es la distancia a la que el electrón se encuentra del núcleo. Esta aclaración es pertinente ya que como es bien sabido el potencial $V(r)=-\frac{e^{2}}{r}$ no es solución de la ecuación de Poisson $\left(\nabla^{2} \phi=-4 \pi \rho\right)$ en dos dimensiones. Sin embargo está fundamentado físicamente por la naturaleza de la interacción entre cargas puntuales.

Este modelo es de interés también en problemas de materia condensada para el cálculo de los estados de un gas bidimensional de electrones confinados bajo la acción de un campo magnético homogéneo [85]. 


\subsection{Atomo de hidrógeno libre en $2 \mathrm{D}$}

Los eigenestados de un átomo de hidrógeno en dos dimensiones se describen por la ecuación de Schrödinger, en coordenadas polares (en unidades atómicas).

$$
\left(-\frac{1}{2}\left(\frac{\partial^{2}}{\partial r^{2}}+\frac{1}{r} \frac{\partial}{\partial r}+\frac{1}{r^{2}} \frac{\partial^{2}}{\partial \phi^{2}}\right)+V(r)\right) \psi(r, \phi)=E \psi(r, \phi)
$$

donde

$$
V(r)=-1 / r
$$

Las soluciones exactas [135] de la ecuación (1.a) son de la forma

$$
\psi_{n l}(r, \phi)=R_{n l}(r) \Phi_{l}(\phi)
$$

siendo la solución angular

$$
\Phi(\phi)=\frac{1}{(2 \pi)^{1 / 2}} e^{i l \phi}, l=0, \mp 1, \mp 2, \mp 3, \ldots,
$$

mientras que las eigenfunciones radiales (normalizadas) para el estado base y los dos primeros estados excitados están dadas por

$$
\begin{gathered}
R_{10}=\beta_{1} e^{-\beta_{1} r / 2}, \\
R_{20}=\left(\beta_{2} / \sqrt{3}\right)\left(1-\beta_{2} r\right) e^{-\beta_{2} r / 2} \\
R_{21}=\left(\beta_{2}^{2} / \sqrt{6}\right) r e^{-\beta_{2} r / 2} .
\end{gathered}
$$

donde 


$$
\beta_{n}=\frac{2}{n-1 / 2}
$$

Mientras que los eigenvalores de la energía están dados por

$$
E_{n}=-\frac{1}{2(n-1 / 2)^{2}}, \text { con } n=1,2,3, \ldots
$$

Siendo $n$ el número cuántico principal. Para una $n$ dada, $|l|=0,1,2, \ldots, n-1$.

\subsection{Atomo de hidrógeno $2 \mathrm{D}$ confinado}

El problema del átomo de hidrógeno en dos dimensiones confinado mediante paredes impentrables, está descrito por la ecuación de Schrödinger (1), donde el potencial $V(r)$ es ahora

$$
V(r)= \begin{cases}+\infty, & r \geq r_{0} \\ -\frac{1}{r}, & r<r_{0}\end{cases}
$$

La funcion de onda que es solución de (1) con el potencial (8) debe satisfacer la condición de frontera

$$
\psi\left(r=r_{0}, \phi\right)=0
$$

Las eigenfunciones de este problema siguen siendo de la misma forma que para el caso libre (ec. 3) y la solución angular es la dada por la ecuación (4).

Las eigenfunciones radiales que ahora llamaremos $R_{n l}^{\prime}$ satisfacen la ecuación radial de Schrödinger en dos dimensiones

$$
-\frac{1}{2}\left(\frac{d^{2} R_{n l}^{\prime}}{d r^{2}}+\frac{1}{r} \frac{d R_{n l}^{\prime}}{d r}-\frac{l^{2}}{r^{2}} R_{n l}^{\prime}\right)+V(r) R_{n l}^{\prime}=E_{n l} R_{n l}^{\prime}
$$


Nótese que, como en el caso libre, la solución $R_{n l}^{\prime}(r)$ depende de $n$ y del valor absoluto de $|l|$. La solución (4) es eigenfunción del momento angular en la dirección $z$.

$$
L_{z}=-i \hbar \frac{\partial}{\partial \phi}
$$

el cual conmuta con el hamiltoniano, por lo que $l$ es un buen número cuántico.

De acuerdo al método variacional directo, la función radial de prueba se escoge como $R_{n l}^{\prime}(r ; \alpha)=R_{n l}(r ; \alpha) \times \chi\left(r ; r_{0}\right)$ donde $R_{n l}(r)$ es la solución radial del problema libre [135], $\alpha$ es un parámetro variacional y $\chi\left(r, r_{0}\right)$ es una función de corte que se anula en $r=r_{0}$. La función $R_{n l}^{\prime}(r ; \alpha)$ claramente satisface la condición de frontera (ec. 9). La función de corte más sencilla es $\chi\left(r ; r_{0}\right)=r_{0}-r$; que es precisamente la que usaremos en este trabajo.

Algunas de las funciones de onda radiales $R_{n l}(r)$ del problema libre se muestran en (5), lo que facilita construir las funciones de onda de prueba para los diferentes niveles energéticos. A continuación se dan explicitamente algunas de las funciones $R_{n l}^{\prime}(r ; \alpha)$.

$$
\begin{gathered}
R_{10}^{\prime}=e^{-\alpha_{1} r}\left(r_{0}-r\right), \\
R_{20}^{\prime}=e^{-\alpha_{2} r}\left(r_{1}\left(\alpha_{2}\right)-r\right)\left(r_{0}-r\right), \\
R_{21}^{\prime}=r e^{-\alpha_{3} r}\left(r_{0}-r\right) .
\end{gathered}
$$

donde $r_{1}\left(\alpha_{2}\right)$ es el nodo de la función radial $R_{20}^{\prime}$, que se determina a partir de la condición de ortogonalidad $<R_{10}^{\prime} \mid R_{20}^{\prime}>=0$. 

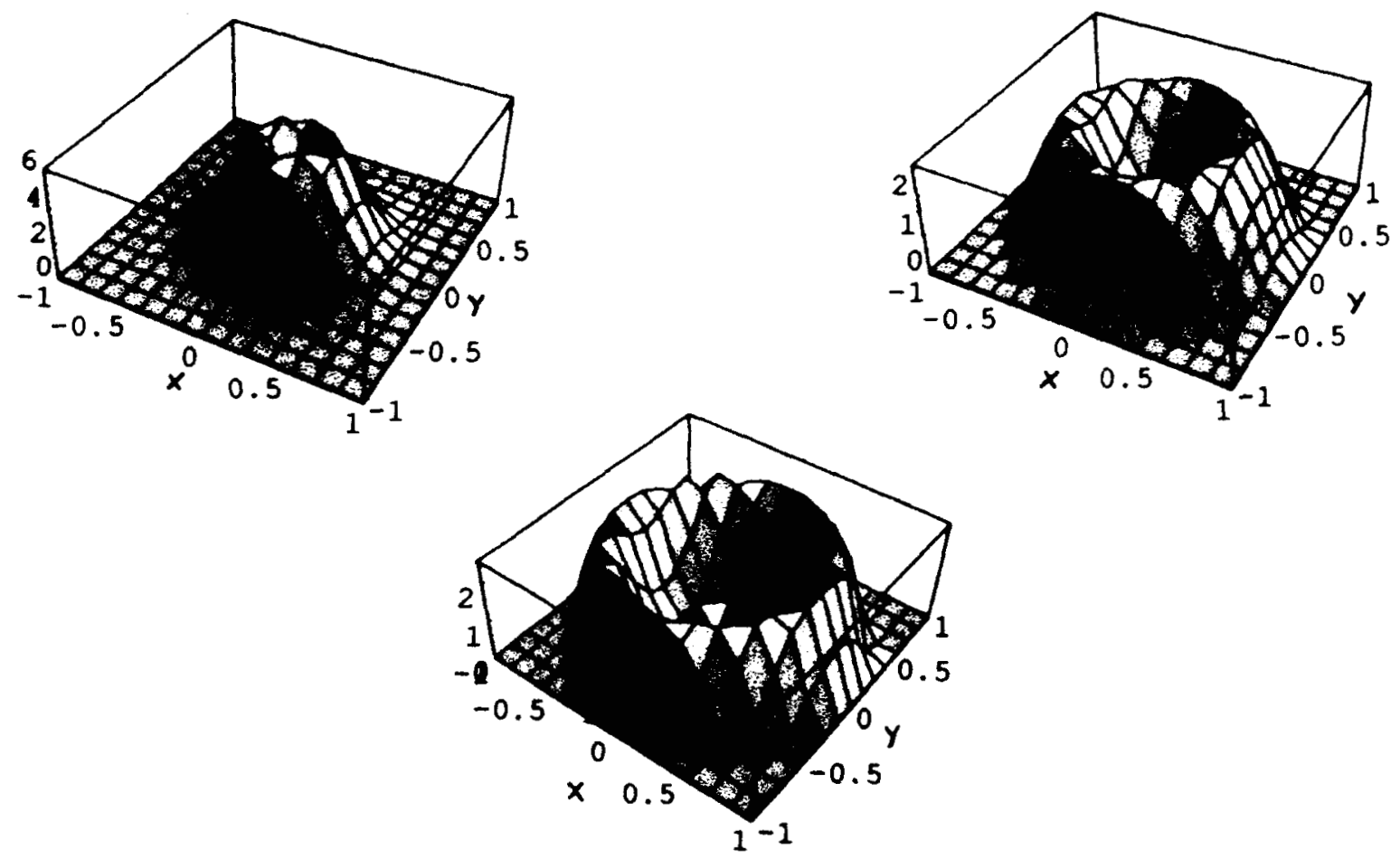

Figura 4.1: Una gráfica de las densidades de probabilidad $|\psi(r, \phi)|^{2}$ para un tamaño de caja $r_{0}=1$ Bohr.

Para tener una idea más clara de como son estos orbitales, en la figura 4.1 se muestran unas gráficas del módulo al cuadrado de las funciones de onda $|\psi(r, \phi)|^{2}$ para un tamaño de caja $r_{0}$ de 1 Bohr. Las gráficas de $|\psi(r, \phi)|^{2}$ representan el sólido de revolución que se obtiene al hacer girar las densidades radiales $D_{n l}(r)$ alrededor de su eje vertical.

\subsubsection{Eigenvalores de la energía}

Las energías óptimas se encuentran minimizando el funcional $E\left(\alpha ; r_{0}\right)$ respecto al parámetro variacional $\alpha$, donde $E\left(\alpha ; r_{0}\right)$ está dada por:

$$
E_{n l}\left(\alpha ; r_{0}\right)=\frac{<R_{n l}^{\prime}|H| R_{n l}^{\prime}>}{<R_{n l}^{\prime} \mid R_{n l}^{\prime}>}
$$


y $H$ es el hamiltoniano del sistema asociado con la ecuación radial de Schrödinger en dos dimensiones (ec. 10).

Tabla 1. Eigenvalores del estado base y algunos estados excitados del átomo de hidrógeno bidimensional confinado. Las distancias están dadas en Bohrs y las energías en hartrees

\begin{tabular}{|c|c|c|c|c|c|c|c|}
\hline $\mathrm{R}$ & $\alpha_{1}$ & $E_{10}$ & $\alpha 3$ & $E_{21}$ & $\alpha 2$ & $r_{1}$ & $E_{20}$ \\
\hline 0.5 & 0.3709 & 3.9259 & 0.9000 & 25.4852 & 0.0383 & 0.1959 & 56.0947 \\
1.0 & 0.8624 & -1.3460 & 0.5812 & 5.2922 & 0.0350 & 0.3651 & 11.3237 \\
2.0 & 1.3595 & -1.9732 & 0.4361 & 0.7659 & 0.2895 & 0.5697 & 1.6515 \\
4.0 & 1.7124 & -1.9994 & 0.4011 & -0.1101 & 0.3893 & 0.7120 & -0.0384 \\
6.0 & 1.8176 & -1.9999 & 0.4335 & -0.2031 & 0.4317 & 0.7500 & -0.1921 \\
8.0 & 1.8663 & -2.0000 & 0.4825 & -0.2184 & 0.4797 & 0.7576 & -0.2163 \\
10.0 & 1.8946 & -2.0000 & 0.5249 & -0.2213 & 0.5222 & 0.7565 & -0.2208 \\
15.0 & 1.9310 & -2.0000 & 0.5825 & -0.2221 & 0.5814 & 0.7527 & -0.2221 \\
20.0 & 1.9487 & -2.0000 & 0.6074 & -0.2222 & 0.6069 & 0.7514 & -0.2222 \\
25.0 & 1.9592 & -2.0000 & 0.6209 & $"$ & 0.6208 & 0.7508 & $"$ \\
30.0 & 1.9661 & -2.0000 & 0.6295 & $"$ & 0.6294 & 0.7506 & $"$ \\
40.0 & 1.9747 & -2.0000 & 0.6397 & $"$ & 0.6395 & 0.7503 & $"$ \\
50.0 & 1.9798 & -2.0000 & 0.6454 & $"$ & 0.6453 & 0.7502 & $"$ \\
60.0 & 1.9831 & -2.0000 & 0.6491 & $"$ & 0.6491 & 0.7501 & $"$ \\
$\infty$ & 2.0000 & -2.0000 & 0.6666 & -0.2222 & 0.6666 & 0.7500 & -0.2222 \\
\hline
\end{tabular}

Las energías y valores de $\alpha$ óptimos se encuentran reportados en la Tabla 1. De esta Tabla podemos observar que cuando el radio de la caja $r_{0} \rightarrow \infty$ los valores de la energía se aproximan a los del átomo bidimensional libre, como es de esperarse. Mientras que cuando $r_{0}$ se hace muy pequeño, la energía se vuelve positiva indicando que la energía cinética se vuelve más grande que la energía potencial, a pesar de que la distancia entre el núcleo y el electrón se vuelve muy pequeña. En este caso, el potencial Coulombiano se vuelve una perturbación ante el efecto del confinamiento y los niveles de energía son similares a los de una partícula confinada en una caja circular.

También es evidente que la degeneración en el momento angular se remueve cuando el sistema se confina dentro del recinto impenetrable y la degeneración vuelve a aparecer solo para tamaños de cajas mayores de 20 Bohrs, como puede observarse de la tabla 1. El estado base del átomo bidimensional libre está más ligado que el estado base de su análogo en tres dimensiones, lo mismo sucede para los otros estados. De igual manera los estados del átomo bidimensional confinado 
poseen energías más bajas que sus análogos de tres dimensiones. Esto puede tener consecuencias de interés práctico al estudiar estados de impurezas hidrogenoides confinadas en la interficie entre dos materiales semiconductores.

Todas las integrales necesarias para evaluar la energía se pueden hacer de manera analítica. Por comodidad decidimos hacer la optimización de la energía numéricamente mediante el método de búsqueda con la sección aurea (golden section search) en una dimensión [95], optimizando al parámetro $\alpha$ hasta una incertidumbre absoluta de 0.0001 .

\subsubsection{Valores esperados de $r$}

Los valores esperados de $r^{k}$ para unos cuantos valores enteros $k$ son de mucha utilidad en el problema tridimensional, porque están relacionados con propiedades físicas del sistema como la presión, la polarizabilidad, el apantallamiento magnético nuclear y el desdoblamiento hiperfino [17, 25, 29]. En el caso bidimensional los valores esperados de $r^{k}$ están dados por

$$
<r_{n l}>=\frac{\int_{0}^{r_{0}} r^{k+1} R_{n l}^{\prime 2} d r}{\int_{0}^{r_{0}} r R_{n l}^{\prime 2} d r}
$$

En la Tabla 2 se reportan los valores esperados $\langle r\rangle,\left\langle r^{2}\right\rangle,\left\langle r^{-1}\right\rangle$ y para $l \neq 0$ también $\left\langle r^{-2}\right\rangle$; el valor $\langle r\rangle$ representa la distancia promedio a la que el electrón se encuentra del núcleo.

La probabilidad de encontrar al electrón dentro del elemento de área $d s=r d r d \phi$ en la vecindad del punto de coordenadas $(r, \phi)$ para cualquier orbital es:

$$
\psi_{n l}^{*}(r, \phi) \psi_{n l}(r, \phi) r d r d \theta
$$

Integrando sobre el ángulo $\phi$ obtenemos la siguiente expresión

$$
N\left(r_{0}, \alpha\right) r{R^{\prime 2}}_{n l}^{2}(r) d r=D_{n l}(r) d r
$$


donde $N\left(r_{0}, \alpha\right)=1 /<R_{n l} \mid R_{n l}>$ es el cuadrado de la constante de normalización.

La ecuación (16) representa la probabilidad de encontrar al electrón entre las distancias $r$ y $r+d r$ del núcleo. Esta misma ecuación sirve para definir a la función de distribución radial de probabilidad $D_{n l}(r)$, que es de mucha utilidad para describir el tamaño de los orbitales.

Tabla 2. Valores esperados de $r$ y $r_{\max }$ para el estado base y algunos estados excitados del átomo de hidrógeno bidimensional confinado. Las distancias están dadas en Bohrs. El valor correspondiente a $r_{0} \rightarrow \infty$ se obtuvo de las fórmulas analíticas de la referencia [135].

\begin{tabular}{|c|c|c|c|c|c|c|c|c|}
\hline$r_{0}$ & $\langle r\rangle$ & $\left\langle r^{2}\right\rangle$ & $\langle 1 / r\rangle$ & $r_{\max }$ & $\langle r\rangle$ & $\left\langle r^{2}\right\rangle$ & $\langle 1 / r\rangle$ & $r_{\max }$ \\
\hline & & $n=1$ & $l=0$ & & $n=2$ & $l=0$ & & \\
0.5 & 0.193 & 0.047 & 8.456 & 0.154 & 0.235 & 0.078 & 11.635 & 0.363 \\
1.0 & 0.335 & 0.147 & 5.147 & 0.138 & 0.518 & 0.350 & 5.036 & 0.716 \\
2.0 & 0.461 & $\mathbf{0 . 3 0 0}$ & 4.092 & 0.259 & 1.114 & 1.472 & 2.047 & 1.309 \\
4.0 & 0.493 & 0.361 & 4.002 & 0.253 & 2.141 & 5.106 & 0.773 & 2.148 \\
6.0 & 0.498 & 0.370 & 4.000 & 0.251 & 2.826 & 8.972 & 0.528 & 2.616 \\
8.0 & 0.499 & 0.372 & 4.000 & 0.254 & 3.200 & 11.716 & 0.462 & 2.789 \\
10.0 & 0.499 & 0.373 & 4.000 & 0.250 & 3.359 & 13.115 & 0.446 & 2.821 \\
15.0 & 0.500 & 0.374 & 4.000 & 0.250 & 3.461 & 14.166 & 0.444 & 2.819 \\
20.0 & 0.500 & 0.375 & 4.000 & 0.250 & 3.482 & 14.412 & 0.444 & 2.805 \\
25.0 & 0.500 & 0.375 & 4.000 & 0.250 & 3.489 & 14.498 & 0.444 & 2.802 \\
30.0 & 0.500 & 0.375 & 4.000 & 0.250 & 3.493 & 14.542 & 0.444 & 2.801 \\
40.0 & 0.500 & 0.375 & 4.000 & 0.250 & 3.496 & 14.583 & 0.444 & 2.800 \\
50.0 & 0.500 & 0.375 & 4.000 & 0.250 & 3.498 & 14.600 & 0.444 & 2.800 \\
60.0 & 0.500 & 0.375 & 4.000 & 0.250 & 3.499 & 14.607 & 0.444 & 2.800 \\
$\infty$ & 0.500 & 0.375 & 4.000 & 0.250 & 3.500 & 14.625 & 0.444 & 2.800 \\
\hline
\end{tabular}

Para un orbital dado la distancia más probable $\left(r_{\max }\right)$ se obtiene al maximizar la correspondiente $D_{n l}$. Para los orbitales con $n-l>0, r_{\max }$ se define como la distancia medida desde el núcleo hasta el máximo principal. Es decir, entre el más externo y más prominente de los máximos. En la Tabla 2 también hemos incluido el valor de $r_{\max }$, para los diferentes valores de $r_{0}$.

Como puede observarse de la Tabla 2, los valores esperados de la posición varían monótonamente ( el mismo comportamiento lo presenta la energía ) y tienden de manera asintótica a los valores del átomo libre, como era de esperarse. El 

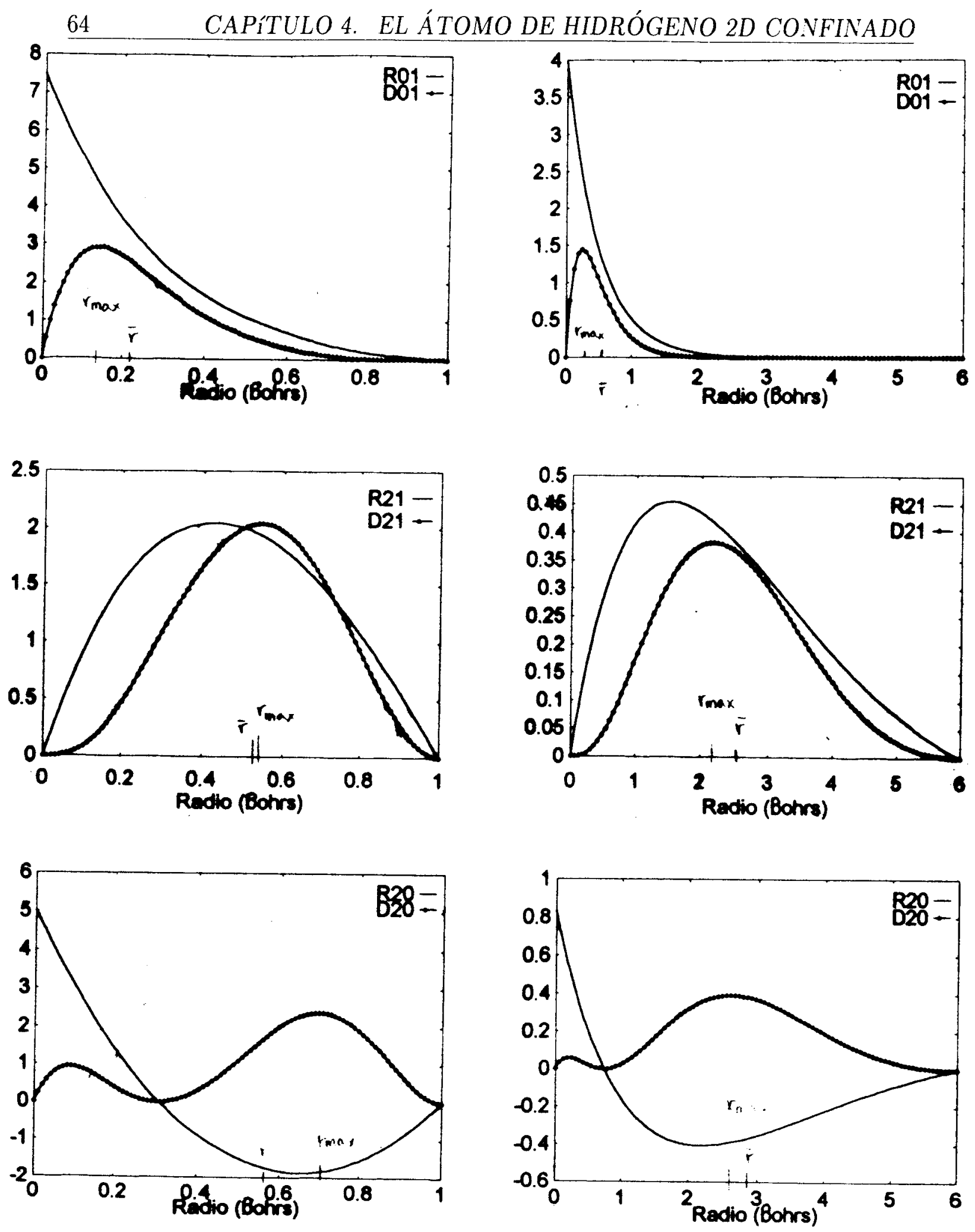

Figura 4.2: Una gráfica de las funciones de onda $R_{n l}(r)$ y las densidades radiales $D_{n l}(r)$ para un tamaño de caja $r_{0}=1,6$. 
comportamiento de $r_{\max }$ no es monótono en general, para ello basta comparar los valores que toma para los estados $n=2, l=0$ y $n=2, l=1$ en función de $r_{0}$. En ambos casos se encuentra un máximo, para un valor de $r_{0}$ cercano a 10 a.u.

Tabla 2. Valores esperados de $r$ y $r_{\max }$ para el estado base y algunos estados excitados del átomo de hidrógeno bidimensional confinado (continuación).

\begin{tabular}{|c|c|c|c|c|c|}
\hline$r_{0}$ & $\langle r\rangle$ & $\left\langle r^{2}\right\rangle$ & $\langle 1 / r\rangle$ & $\left\langle 1 / r^{2}\right\rangle$ & $r_{\max }$ \\
\hline & & $n=2$ & $l=1$ & & \\
0.5 & 0.272 & 0.082 & 4.274 & 23.416 & 0.278 \\
1.0 & 0.535 & 0.318 & 2.180 & 6.136 & 0.542 \\
2.0 & 1.034 & 1.195 & 1.140 & 1.705 & 1.026 \\
4.0 & 1.885 & 4.047 & 0.641 & 0.558 & 1.767 \\
6.0 & 2.473 & 7.134 & 0.503 & 0.356 & 2.161 \\
8.0 & 2.778 & 9.212 & 0.460 & 0.305 & 2.282 \\
10.0 & 2.898 & 10.207 & 0.449 & 0.295 & 2.291 \\
15.0 & 2.972 & 10.932 & 0.445 & 0.295 & 2.269 \\
20.0 & 2.987 & 11.103 & 0.444 & 0.295 & 2.260 \\
25.0 & 2.993 & 11.168 & 0.444 & 0.296 & 2.256 \\
30.0 & 2.995 & 11.194 & 0.444 & 0.296 & 2.254 \\
40.0 & 2.997 & 11.217 & 0.444 & 0.296 & 2.252 \\
50.0 & 2.998 & 11.231 & 0.444 & 0.296 & 2.251 \\
60.0 & 2.999 & 11.238 & 0.444 & 0.296 & 2.251 \\
$\infty$ & 3.000 & 11.250 & 0.444 & 0.296 & 2.250 \\
\hline
\end{tabular}

En la figura 4.2 se muestran las gráficas de las funciones de onda $R_{n l}$ y las densidades radiales $D_{n l}$, de los tres primeros estados del átomo de hidrógeno bidimensional confinado. Es decir, el estado base y los dos primeros estados excitados para $r_{0}=1,6$ Bohrs. En esta figura también se muestra la posición de $\bar{r}=\langle r\rangle$ y $r_{\max }$ para cada caso.

Todos los cálculos de esta sección (así como de la anterior) se pueden hacer en forma analítica y las evaluaciones fueron efectuadas numéricamente. La evaluación numérica fue hecha en una microcomputadora 386-DX. El tiempo consumido fue de alrededor de 1 segundo en la optimización de la energía y mucho menos de un segundo para el cálculo de los valores esperados de la distancia, para cada valor de $r_{0}$. 


\subsection{El método de las series}

El método de las series que hemos empleado en el capítulo 2 y 3 , también puede emplearse para encontrar valores de alta precisión para este problema. La aplicación del método ya ha sido discutida antes, para el átomo de hidrógeno tridimensional. El presente problema no presenta dificultades adicionales, así que solo nos concretaremos a presentar los resultados. En la tabla 3 mostramos los eigenvalores de la energía determinados por el método de las series, las cifras que se muestran en esta tabla estan redondeadas en la última cifra.

Tabla 3. Eigenvalores de alta precisión para el estado base y los dos primeros estados excitados como función del tamaño de la caja. Las distancias estan en Bohrs, mientras que las energias se encuentran expresadas en hartrees.

\begin{tabular}{|c|c|c|c|}
\hline$r_{0}$ & $E_{10}$ & $E_{20}$ & $E_{21}$ \\
\hline 0.5 & 3.907318370054 & 52.069123931538 & 25.213211091985 \\
1.0 & -1.349785716814 & 10.758194353019 & 5.242787506960 \\
2.0 & -1.981158591165 & 1.607823563313 & 0.760782846776 \\
4.0 & -1.999986566533 & -0.038145687612 & -0.110082289910 \\
6.0 & -1.999999993068 & -0.192827805360 & -0.203804143840 \\
8.0 & -1.999999999996 & -0.217363859239 & -0.219239253323 \\
10.0 & -1.999999999999 & -0.221500051056 & -0.221793574951 \\
15.0 & -1.999999999999 & -0.222218547342 & -0.222220171224 \\
20.0 & -2.000000000000 & -0.222222210221 & -0.222222215721 \\
25.0 & -2.000000000000 & -0.222222222191 & -0.222222222206 \\
30.0 & -2.000000000000 & -0.222222222222 & -0.222222222222 \\
40.0 & -2.000000000000 & -0.222222222222 & -0.222222222222 \\
$\infty$ & -2.000000000000 & -0.222222222222 & -0.222222222222 \\
\hline
\end{tabular}

Si comparamos la tabla 3 con la tabla 1 , vemos que los valores reportados en la tabla 1 solo tienen dos decimales de precisión para radios pequenõs, sin embargo, para tener una idea cualitativa del comportamiento de la energía como función del radio de encajonamiento, ésta es suficiente. Los valores reportados en la tabla 3 pueden servir como una referencia para determinar la precisión de otros métodos, que se utilicen para el estudio de este problema. 


\subsection{Conclusiones}

En el problema del átomo de hidrógeno en dos y tres dimensiones (libres y confinados) existen semejanzas y diferencias importantes. Empecemos analizando el caso libre. Lo primero que llama la atención es que al reemplazar al número cuántico principal $n$ por $n-\frac{1}{2}$, en la fórmula para la energía del átomo de hidrógeno en 3 dimensiones, se obtiene el espectro de energías para el caso bidimensional, así como otras fórmulas importantes [135]. La diferencia importante en el caso de dos dimensiones es que las funciones propias solo tienen una parte angular que va como $e^{i l \phi}$ y no los armónicos esféricos, así que las funciones de onda para el caso bidimensional no se obtienen trivialmente (haciendo $\phi=\pi / 2$ ) de las soluciones del caso de tres dimensiones. Sin embargo las densidades radiales $D_{n l}(r)$ del átomo bidimensional (libre y confinado) tienen la misma forma que la de su análogo en tres dimensiones, aunque las dimensiones espaciales son diferentes. Este hecho nos indica que la carga electrónica esta más localizada en un caso que en el otro.

El átomo de hidrógeno bidimensional libre se encuentra más ligado que su análogo en tres dimensiones. El mismo comportamiento de los eigenvalores de la energía se encuentra en los sistemas confinados, es decir, para un tamaño de caja dado $r_{0}$ la energía del átomo de hidrógeno bidimensional es menor que la correspondiente energía del átomo en tres dimensiones.

Observando la tabla 1 vemos que la energía del estado base del átomo bidimensional confinado es casi igual a la del átomo (bidimensional) libre para un valor de $r_{0}=4$ Bohrs, mientras que para el átomo de tres dimensiones confinado el correspondiente radio $r_{0}$ es de 8 Bohrs (ver las Tabla 2 y 4 del capítulo 3 ). Mientras que para los estados $E_{21}$ y $E_{20}$ del átomo de dos dimensiones el valor de $r_{0}$, para el cual se tiene la mayor parte de la energía del caso libres es 10 Bohrs, los correspondientes valores para el átomo de tres dimensiones dan un valor cercano a 12 Bohrs.

El método de las series aplicado a este problema proporciona eigenvalores de la energía y eigenfunciones de alta precisión. Al comparar éstos con los obtenidos 
mediante el método variacional directo, vemos que este último sólo proporciona, valores cuyos errores son del orden de centésimas o milésimas de hartree, suficiente para entender el comportamiento de los niveles de energía como función del radio de confinamiento.

Aún cuando en la realidad no existe un sistema cuántico estrictamente bidimensional, el modelo del átomo de hidrógeno $2 \mathrm{D}$ confinado constituye un caso límite importante para el estudio de impurezas hidrogenoides confinadas en discos y alambres cuánticos. Los cuales corresponden muy cercanamente a estructuras consistentes en arreglos de alambres cuánticos de silicio (silicio poroso), e impurezas hidrogenoides o excitones atrapados en zonas espacialmente acotadas en la interficie entre dos materiales semiconductores.

En este trabajo hemos considerado el problema de un átomo de hidrógeno en dos dimensiones confinado mediante paredes impenetrables, en una segunda etapa consideramos resolver el problema introduciendo un campo externo uniforme, ya sea eléctrico o magnético. 


\section{Capítulo 5}

\section{La inversión del $\mathrm{NH}_{3}$ y sus especies isotópicas}

\subsection{Introducción}

La molécula del $\mathrm{NH}_{3}$ tiene forma de una pirámide triangular, con los tres hidrógenos en su base y el átomo de nitrógeno en la arista. La inversión ocurre cuando el átomo de nitrógeno oscila de un lado a otro del plano de los hidrógenos (fig. 5.1), es un viejo problema que se ha estudiado extensämente mediante métodos experimentales y empíricos [141-152], Los estudios a través de métodos ab initio $[83-84,153]$ han sido escasos.

Para representar la barrera de inversión del $\mathrm{NH}_{3}$ se han propuesto diversos tipos de potenciales con doble pozo [143,148-149, 83-84, 152-153], pero tal vez la forma analítica más sencilla para representar este potencial sea la de un oscilador anarmónico cuártico con doble pozo simétrico [84]:

$$
V(x)=-\frac{1}{2} x^{2}+\lambda x^{4}, \lambda>0
$$

El espectro energético consiste de dobletes, un miembro de cada doblete pertenece a un estado simétrico y el otro a uno antisimétrico. Una característica de este 


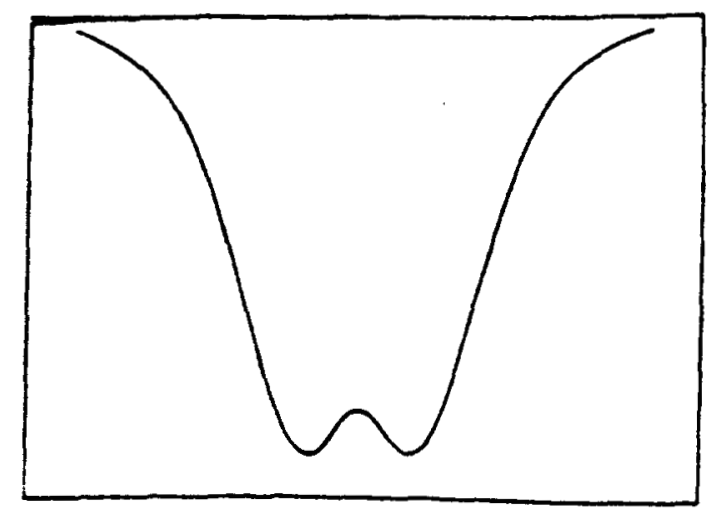

Figura 5.1: El potencial de doble pozo de la inversión de la molécula de $\mathrm{NH}_{3}$.

potencial, es que la ecuación de Schrödinger correspondiente no puede resolverse por medio de teoría de perturbaciones [154-155], ya que la serie perturbativa en potencias del parámetro $\lambda$ es divergente para todo valor de $\lambda>0$.

Hace algunos años Bunker y colaboradores [153] calcularon la superficie de energía para el $\mathrm{NH}_{3}$, mostrando que la barrera de inversión puede predecirse a partir de primeros principios. Campoy y colaboradores [83] seleccionaron algunos puntos de la superficie de energía calculada por Bunker [153] y ajustando por mínimos cuadrados encontraron un polinomio de octavo grado para representar el potencial de inversión. En este trabajo se propone un polinomio de cuarto grado (que reproduce satisfactoriamente los puntos de la superficie de energía seleccionados por Campoy [83]) y a partir de este polinomio se encuentra el potencial del oscilador anarmónico cuártico de doble pozo, posteriormente se encuentran las constantes moleculares y las frecuencias de la inversión del $\mathrm{NH}_{3}$. El polinomio de cuarto grado se determina mediante un ajuste de mínimos cuadrados de algunos puntos de la superficie de potencial a lo largo de la coordenada de inversión. En este trabajo no empleamos ningun dato de la geometría experimental (como en trabajos anteriores $[83,154]$ ) para determinar las frecuencias de inversón. Todo se hace a partir de primeros principios. 


\subsection{El potencial anarmónico cuártico}

Proponemos que el potencial de inversión $\mathrm{V}(\mathrm{x})$ sea representado por un polinomio de cuarto grado :

$$
V^{\prime \prime}(x)=c_{0}+c_{1} x^{2}+c_{2} x^{4}
$$

Para hacer más sencillo el cálculo definimos un nuevo potencial $\mathrm{V}(\mathrm{x})$ mediante:

$$
V^{\prime}(x)=V^{\prime \prime}(x)-c_{0}
$$

Si además definimos

$$
a=-2 c_{1} \text { y } b=2 c_{2}
$$

el hamiltoniano respectivo queda de la siguiente manera:

$$
H^{\prime}=\frac{p^{2}}{2 \mu}-\frac{1}{2} a x^{2}+\frac{1}{2} b x^{4}
$$

donde $\mu$ es la masa reducida del sistema.

Este es un problema que aparentemente tiene dos parámetros, pero realmente es un problema de un solo parámetro como a continuación lo veremos. Hagamos el siguiente cambio de variables para tener el hamiltoniano en unidades adimensionales:

$$
P=\left(\frac{\hbar^{2}}{\mu a}\right)^{1 / 4} p \text { y } Q=\left(\frac{\mu a}{\hbar^{2}}\right)^{1 / 4} x
$$

El hamiltoniano se reduce al de un oscilador anarmónico cuártico de doble pozo:

$$
H=\frac{1}{2} P^{2}-\frac{1}{2} Q^{2}+\lambda Q^{4}
$$


la unidad de la energía es ahora

$$
\epsilon=\hbar \nu_{0}=\left(\frac{a \hbar^{2}}{\mu}\right)^{1 / 2}
$$

mientras que la constante anarmónica $\lambda$ es:

$$
\lambda=\frac{b \epsilon}{2 a^{2}}
$$

\subsection{Método de series}

Como vimos en la sección anterior, el potencial representado mediante un polinomio de cuarto grado (ec. 1) se reduce a un potencial anarmónico cuártico con doble pozo (ec. 6). La búsqueda de soluciones tanto numéricas como analíticas a los potenciales cuárticos ha sido un campo de intensa investigación desde hace más de 30 años (vea un resumen en [158]). El problema no admite solución en el marco de la teoría de perturbaciones estándar, ya que la serie perturbativa de la energía que es expresada en términos del parámetro anarmónico $\lambda$, diverge para cualquier valor positivo de $\lambda$.

El problema de encontrar las eigenfunciones y eigenvalores de este problema, ha generado dos vertientes principales: La primera de ellas esta basada en introducir correcciones a la teoría de perturbaciones usual, mientras que la segunda esta basada en métodos no perturbativos.

A continuación emplearemos el método de la sección 1.2 que ya hemos empleado en los capítulos 2 y 3 , que es un método no perturbativo con el cual se obtienen eigenvalores de alta precisión.

La aplicación del método de encajonamiento al oscilador anarmónico cuártico es muy sencilla y ya había sido empleada por Campoy y Palma [78], y es como sigue:

La ecuación de Schrödinger correspondiente al hamiltoniano de la ecuación (6) se puede escribir como: 


$$
\psi_{n}{ }^{\prime \prime}(x)=\left(x^{2}-2 \lambda x^{4}-2 E_{n}\right) \psi_{n},
$$

donde el símbolo' indica derivada respecto a $x$, y $E_{n}$ son los eigenvalores de la energía. En este caso particular tenemos un potencial simétrico, por lo que las eigenfunciones deberán tener simetría definida, esto facilita el cálculo porque en el desarrollo en serie de Taylor sólo habrá potencias pares o impares. Procediendo como en el capítulo 2 y 3 tenemos:

$$
\psi(x)=\sum_{k} T_{k}(x)
$$

donde

$$
T_{k}=\left(\psi^{(k)}(0) / k !\right) x^{k}
$$

De donde se obtiene la siguiente relación de recurrencia para las derivadas de la función $\psi(x)$, evaluadas en el origen

$$
T_{p+2}=\frac{-2 E_{n} T_{p}+T_{p-2}-2 \lambda T_{p-4}}{(p+1)(p+2)} .
$$

La derivada parcial de la función de onda esta dada por

$$
\partial \psi / \partial E_{n}=\sum_{m} \partial T_{m} / \partial \epsilon=\sum_{p} \dot{T}_{p}
$$

Derivando (10) respecto a $E_{n}$, se obtiene

$$
\dot{T}_{p+2}=\frac{-2 E_{n} \dot{T}_{p}+\dot{T}_{p-2}-2 \lambda \dot{T}_{p-4}-2 T_{p}}{(p+1)(p+2)}
$$

con lo cual queda tederminada la ec. (11), el punto significa derivada parcial respecto a la energía. 
Dada la simetría del potencial, las condiciones iniciales se pueden escoger sin pérdida de generalidad como:

$$
\begin{gathered}
\psi_{n}(0)=1 \psi_{n}^{\prime}=0 \text { si } n \text { es par } \\
\psi_{n}(0)=0 \psi_{n}^{\prime}=1 \text { si } n \text { esimpar. }
\end{gathered}
$$

Usando las ecuaciones (9a), (11) y las condiciones iniciales (13) en:

$$
\epsilon_{i+1}=\epsilon_{i}-\psi\left(x_{0}, \epsilon_{i}\right) /\left[\partial \psi\left(x_{0}, \epsilon_{i}\right) / \partial \epsilon\right]
$$

se obtienen los valores de $E_{n}$ para cada valor de $\lambda$ y cada ancho de la caja de confinamiento. En este caso nos interesan solo los valores obtenidos para cajas muy grandes (valores asintóticos).

Los eigenvalores de la ecuación (8) que se obtienen por este método se comparararon con los obtenidos por Aquino [158] por un método independiente basado en las propiedades asintóticas de la función de onda, encontrándose en ambos casos los mismos resultados. El método alternativo empleado para dar solución al oscilador anarmónico cuártico esta basado sobre las propiedades asintóticas de las funciones de onda y se encuentra extensamente discutido en la referencia [158].

Tabla 1. Puntos seleccionados de la superficie de energía calculada por Bunker [153].

\begin{tabular}{|c|c|c|c|}
\hline $\mathrm{R}$ (a. u.) & $\alpha$ & $\bar{x}$ (a. u. $)$ & $E($ a. u. $)$ \\
\hline 1.8878 & 120 & 0.0 & -56.406388 \\
1.89 & 113 & \pm 0.51011 & -56.413044 \\
1.95 & 113 & \pm 0.52630 & -56.411837 \\
1.95 & 106 & \pm 0.75416 & -56.414996 \\
1.95 & 100 & \pm 0.90956 & -56.413357 \\
1.95 & 95 & \pm 1.02302 & -56.408752 \\
\hline
\end{tabular}




\subsection{Espectro de inversión del $\mathrm{NH}_{3}$}

La inversión del $\mathrm{NH}_{3}$ se puede tratar con buena aproximación como un problema de una partícula en un potencial unidimensional $V(x)$, en donde $x$ representa a la coordenada de inversión, es decir $x$ mide la distancia del átomo de $\mathrm{N}$ al plano de los hidrógenos, donde ambas cantidades $(V(x)$ y $x)$ están dadas en unidades atómicas. Si $R$ representa la distancia del nitrógeno a uno de los hidrógenos y $\alpha$ es el ángulo formado entre los enlaces H-N.H, entoces la coordenada de inversión $x$ estará dada por:

$$
x=\left(1-\frac{4}{3} \sin ^{2}(\alpha / 2)\right)^{1 / 2} R=R \sin \beta
$$

Donde $R$ es la distancia del enlace $\mathrm{N}-\mathrm{H}, \beta$ es el ángulo entre el enlace $\mathrm{N}-\mathrm{H}$ y el plano de los hidrógenos. Si $m$ y $M$ son las masas de los átomos de hidrógeno y nitrógeno respectivamente, la masa reducida se puede expresar como $\mu=3 \mathrm{~m}(\mathrm{M}+$ $\left.3 m \sin ^{2} \beta\right) /(M+3 m)$. En este trabajo a diferencia de los anteriores [83,154], el ángulo $\beta$ no se ha tomado igual a 2149 que corresponde al valor de la configuración de equilibrio (experimental) [151], sino que se ha calculado mediante interpolación de los datos de la Tabla 1 , encontrándose que $\beta$ para la configuración de equilibrio es igual a 2244 .

Como ya se había mencionado en la introducción existe, un buen número de propuestas para la forma analítica de $V(x)$. En este trabajo se propone usar como potencial de inversión un polinomio de cuarto grado, que reproduzca satisfactoriamente los puntos de la superficie de potencial. Esto se logra tomando el mismo conjunto de puntos seleccionados por Campoy et al [83]; este conjunto forma parte de un total de 54 puntos que fueron calculados mediante métodos ab initio por Bunker [153]. Los puntos de la superficie de energía que fueron seleccionados están reportados en la Tabla 1.

El polinomio de cuarto grado (ec. 2.1) que mejor reproduce los datos de la Tabla 1 es el siguiente: 
Tabla 2. Valores de las constantes moleculares del $\mathrm{NH}_{3}$. La referencia [150] es el mejor cálculo empírico que se ha realizado; en éste se uso un potencial de la forma $V(q)=a q^{2} / 2+b q^{4} / 2+\operatorname{dexp}\left(-c q^{2}\right), \mathrm{y}$ los parámetros $(\beta, \mathrm{a}, \mathrm{b}, \mathrm{c} \mathrm{y} \mathrm{d})$ se variaban hasta obtener el valor más cercano al reportado experimentalmente. El valor experimental para el alto de la barrera y la distancia de equilibrio son [145] $(2020 \pm 12) \mathrm{cm}^{-1} \mathrm{y}$ $(0.382 \pm 0.004) \dot{A}$, respectivamente

\begin{tabular}{|c|c|c|}
\hline Constante & Este trabajo & Ref. [150] \\
\hline$\mu(\mathrm{g})$. & $4.263 \times 10^{-24}$ & $4.263 \times 10^{-24}$ \\
$\mathrm{a}($ dinas $/ \mathrm{cm})$ & $9.45 \times 10^{4}$ & $9.42 \times 10^{4}$ \\
$\mathrm{~b}\left(\right.$ dinas $\left./ \mathrm{cm}^{3}\right)$ & $2.96 \times 10^{21}$ & $0.47 \times 10^{21}$ \\
$\mathrm{c}\left(\mathrm{cm}^{-2}\right)$ & 0.00 & $4.269 \times 10^{16}$ \\
$\mathrm{~d}(\mathrm{ergs})$ & 0.00 & $2.452 \times 10^{-12}$ \\
$\nu_{0}\left(\mathrm{~cm}^{-1}\right)$ & 790.3 & 789.9 \\
$\mathrm{H}\left(\right.$ alto barrera $\left.\mathrm{cm}^{-1}\right)$ & 1894.5 & 2018 \\
$X_{e}($ dist. equilibrio $\dot{\mathrm{A}})$ & 0.399 & 0.382 \\
$\beta$ & $22^{\circ} 44^{\prime}$ & $22^{\bullet} 12^{\prime}$ \\
\hline
\end{tabular}

$$
V^{\prime \prime}(x)=c_{0}+c_{1} x^{2}+c_{2} x^{4}
$$

donde $c_{0}=-56.4064$ hartrees, $c_{1}=-3.034493 \times 10^{-2}$ hartrees $/ \dot{\mathrm{A}}^{2}$ y $c_{2}=$ $2.666604 \times 10^{-2}$ hartrees $/ \dot{\mathrm{A}}^{4}$. Con estos datos se encuentra el valor de la constante anarmónica $\lambda$ (ec. 7b), la unidad de la energía $\epsilon$ (ec. 7a) y algunos otros valores de las constantes moleculares del $\mathrm{NH}_{3}$ que están resumidas en la Tabla 2.

Tabla 3. Eigenvalores de la energía del hamiltoniano asociado al potencial de inverión (ec. 1) para el $\mathrm{NH}_{3}$ y sus especies isotópicas, en unidades de $\times 10^{-13}$ ergs.

\begin{tabular}{|c|c|c|c|c|}
\hline Estado & $\mathrm{NH}_{3}$ & $\mathrm{NH}_{2} \mathrm{D}$ & $\mathrm{NHD}_{2}$ & $\mathrm{ND}_{3}$ \\
& $\lambda=0.02607$ & $\lambda=0.02354$ & $\lambda=0.02152$ & $\lambda=0.01970$ \\
\hline $\mathrm{E}_{0}$ & -2.6998882 & -2.7679426 & -2.8299008 & -2.9499003 \\
$\mathrm{E}_{1}$ & -2.6979627 & -2.7633429 & -2.8296371 & -2.9498072 \\
$\mathrm{E}_{2}$ & -0.8547673 & -1.0573977 & -1.2425126 & -1.4451772 \\
$\mathrm{E}_{3}$ & -0.7504095 & -1.0573977 & -1.2218299 & -1.4365080 \\
$\mathrm{E}_{4}$ & 0.4015411 & 0.1125399 & 0.0905031 & -0.2796931 \\
$\mathrm{E}_{5}$ & 1.1010145 & 0.5857529 & 0.2217558 & -0.0918034 \\
$\mathrm{E}_{6}$ & 2.1385022 & 1.4609084 & 1.0046286 & 0.6479646 \\
$\mathrm{E}_{7}$ & 3.2563685 & 2.3721658 & 1.7657043 & 1.2762110 \\
\hline
\end{tabular}


Como se mostró en la sección 2, el problema con un potencial como el de la ec. 2.1 se reduce al de un oscilador anarmónico de doble pozo. Los eigenvalores de la ec. 2.1 están dados en la Tabla 3, mientras que en las tablas 4 se encuentran reportados los valores del espectro vibracional del $\mathrm{NH}_{3}$ que predice este modelo, junto con los valores experimentales [145] y los cálculos realizados por Palma et al [83]'y por Bunker et al [153].

Tabla 4. Espectro vibracional del $\mathrm{NH}_{3}$ a lo largo de la coordenada de inversión en $\mathrm{cm}^{-1}, \mathrm{~s}=$ simétrico y a $=$ anti-simétrico

\begin{tabular}{|c|c|c|c|c|}
\hline Estado & Ref. [153] & Ref.[83] & Presente trabajo [84] & Experimental [145] \\
\hline $\mathrm{O}^{s}$ & 0.0 & 0.0 & 0.0 & 0.0 \\
$\mathrm{O}^{a}$ & 1.02 & 0.995 & 0.969 & 0.793 \\
$1^{s}$ & 986.7 & 924.12 & 928.92 & 932.51 \\
$1^{a}$ & 1032.3 & 981.04 & 981.46 & 968.12 \\
$2^{s}$ & 1682.0 & 1559.57 & 1561.41 & 1597.60 \\
$2^{a}$ & 2011.6 & 1931.34 & 1913.56 & 1882.18 \\
$3^{s}$ & & 2473.40 & 2435.88 & 2384.17 \\
$3^{a}$ & & 3065.91 & 2998.67 & 2895.61 \\
\hline
\end{tabular}

Aunque solo hemos tomado 6 de los puntos de la superficie de potencial calculada por Bunker para obtener $V(x)$ mediante un ajuste con minimos cuadrados, vemos de la tablas 4 y 5 que se pueden obtener resultados con muy buena precisión.

Tabla 5. Espectro vibracional del $\mathrm{ND}_{3}$ a lo largo de la coordenada de inversión en $\mathrm{cm}^{-1}, \mathrm{~s}=$ simétrico y $\mathrm{a}=$ anti-simétrico

\begin{tabular}{|c|c|c|c|}
\hline Estado & Ref.[14] & Presente trabajo & Experimental [145] \\
\hline $\mathrm{O}^{s}$ & 0.0 & 0.0 & 0.0 \\
$\mathrm{O}^{a}$ & 0.048 & 0.047 & 0.053 \\
$1^{s}$ & 756.01 & 757.55 & 745.7 \\
$1^{a}$ & 760.84 & 761.91 & 749.4 \\
$2^{s}$ & 1338.91 & 1344.31 & 1359 \\
$2^{a}$ & 1442.98 & 1438.90 & 1429 \\
$3^{s}$ & 1818.64 & 1811.33 & 1830 \\
$3^{a}$ & 2151.52 & 2127.62 & 2106.6 \\
\hline
\end{tabular}

\subsection{Especies isotópicas del $\mathrm{NH}_{3}$}

A partir de la aproximación de Born-Oppenheimer la misma curva de potencial del $\mathrm{NH}_{3}$ puede usarse para las especies isotópicas $\mathrm{ND}_{3}, \mathrm{NH}_{2} \mathrm{D}$ y $\mathrm{NHD}_{2}$ y los 
78CAPÍTULO 5. LA INVERSIÓN DEL $\mathrm{NH}_{3}$ Y SUS ESPECIES ISOTÓPICAS

resultados deben de ser también satisfactorios ya que el núcleo del deuterio es más pesado que el del hidrógeno. De la Tabla 5 podemos observar que los resultados del presente trabajo son muy parecidos a los que se obtienen al usar un polinomio de octavo grado [83] como potencial de la inversión.

Los resultados obtenidos para las especies isotópicas mezcladas, el $\mathrm{NH}_{2} \mathrm{D}$ y el $\mathrm{NHD}_{2}$, se muestran en la Tabla 6; en estos casos el movimiento es más complicado, pero continuaremos suponiendo que la superficie de potencial es la misma que la del $\mathrm{NH}_{3}$. Nuestros resultados los comparamos con algunos valores experimentales en la Tabla 7, por ejemplo para la separación del estado base $\Delta_{0}=\left|O^{a}-O^{s}\right|$ y para la separación del primer estado excitado $\Delta_{1}=\left|1^{a}-1^{s}\right|$, además en dicha Tabla se encuentra reportado el valor de $k$, que es igual a la raíz cuadrada de la razón de la masa reducida de la especie en particular a la masa reducida del $\mathrm{NH}_{3}$.

Tabla 6. Espectro vibracional del $\mathrm{NH}_{2} \mathrm{D}$ y del $\mathrm{NHD}_{2}$ a lo largo de la coordenada de inversión en $\mathrm{cm}^{-1}, \mathrm{~s}=$ simétrico $\mathrm{y} \mathrm{a}=$ anti-simétrico

\begin{tabular}{|c|c|c|}
\hline Estado & $\mathrm{NH}_{2} \mathrm{D}$ & $\mathrm{NHD}_{2}$ \\
\hline $\mathrm{O}^{s}$ & 0.0 & 0.0 \\
$\mathrm{O}^{a}$ & 0.342 & 0.133 \\
$1^{s}$ & 854.16 & 799.17 \\
$1^{a}$ & 877.32 & 809.58 \\
$2^{s}$ & 1443.16 & 1379.14 \\
$2^{a}$ & 1681.40 & 1536.35 \\
$3^{s}$ & 2122 & 1930.49 \\
$3^{a}$ & 2580.77 & 2313.36 \\
\hline
\end{tabular}

Los valores experimentales de $\Delta_{0}$ y $\Delta_{1}$ para el $\mathrm{NH}_{2} \mathrm{D}$ y el $\mathrm{NHD}_{2}$ han sido tomados de las referencias [150] y [157]. De la Tabla 7 también podemos observar que el potencial de cuarto grado que se ha utilizado en este trabajo produce en general mejores resultados que los potenciales de octavo grado $[83,153]$.

\subsection{Conclusiones}

Los niveles de energía del $\mathrm{ND}_{3}$ no son particularmente sensibles al tipo del potencial usado para modelar la inversión, y esto se puede observar en la Tabla 5 
donde se muestran los resultados con el potencial polinomial de cuarto grado y los reportados en la referencia [83] donde se usa un polinomio de octavo grado, puede observarse que ambos resultados son muy parecidos; creemos que los resultados para las moléculas $\mathrm{NH}_{2} \mathrm{D}$ y $\mathrm{NHD}_{2}$ siguen el mismo comportamiento, mientras que en el caso del $\mathrm{NH}_{3}$ se observan diferencias significativas con los resultados de la referencia [83], siendo los de este trabajo [84] en general más cercanos a los valores experimentales.

Tabla 7. Energías de separación para $\mathrm{NH}_{3}, \mathrm{ND}_{3}, \mathrm{NH}_{2} \mathrm{D}$ y $\mathrm{NHD}_{2}$ en $\mathrm{cm}^{-1}$.

$$
\text { ( } s=\text { simétrico, } a=\text { anti-simétrico) }
$$

\begin{tabular}{|c|c|c|c|c|}
\hline & Experimental & Calculado & Ref. [83] & Ref. [148] \\
\hline & & $\mathrm{NH}_{3}$ & & \\
\hline$\Delta_{0}$ & 0.793 & 0.969 & 0.995 & 1.02 \\
\hline$\Delta_{1}$ & 35.81 & 52.54 & 56.92 & 45.6 \\
\hline $1^{s}$ & 932.51 & 928.92 & 924.12 & 986.7 \\
\hline $1^{a}$ & 968.32 & 981.46 & 981.04 & 1032.3 \\
\hline$k$ & & 1.0 & & \\
\hline & & $\mathrm{ND}_{3}$ & & \\
\hline$\Delta_{0}$ & 0.053 & 0.047 & 0.048 & \\
\hline$\Delta_{1}$ & 3.7 & 4.36 & 4.83 & \\
\hline $1^{s}$ & 745.7 & 757.55 & 756.01 & \\
\hline $1^{a}$ & 749.4 & 762.91 & 760.84 & \\
\hline$k$ & & 1.323 & & \\
\hline & & $\mathrm{NH}_{2} \mathrm{D}$ & & \\
\hline$\Delta_{0}$ & 0.406 & 0.342 & & \\
\hline$\Delta_{1}$ & 19.7 & 23.16 & & \\
\hline $1^{s}$ & 874 & 854.16 & & \\
\hline $1^{a}$ & 894 & 877.32 & & \\
\hline$k$ & & 1.128 & & \\
\hline & & $\mathrm{NHD}_{2}$ & & \\
\hline$\Delta_{0}$ & 0.1705 & 0.133 & & \\
\hline$\Delta_{1}$ & 9.84 & 10.41 & & \\
\hline $1^{s}$ & 808 & 799.17 & & \\
\hline $1^{a}$ & 818 & 809.58 & & \\
\hline$k$ & & 1.233 & & \\
\hline
\end{tabular}

La discrepancia entre nuestros valores calculados (especialmente en los niveles de mayor energia) y los valores experimentales puede deberse al hecho de que el polinomio de cuarto grado crece indefinidamente cuando se incrementa la coordenada de inversión $x$, mientras que el potencial verdadero tiende a la disociación molecular para cierto valor de $x$ (figura 1 ). 
Los puntos del potencial calculados por primeros principios determinan completamente las observables del problema (las constantes moleculares y el espectro de inversión), así que en el presente trabajo no ha sido necesario usar ningún valor experimental de la geometría ni parámetros ajustables para obtener las observables.

Las constantes moleculares encontradas con este modelo son muy similares a las obtenidas por Swalen e Ibers [150] con un método totalmente empírico.

El encajonar al potencial de la inversión del amoniaco es tan sólo un artificio que es particularmente útil para resolver la ecuación de Schrödinger correspondiente. El objetivo de este procedimiento es el de mantener un alto grado de precisión en la obtención de los eigenvalores del sistema libre, a través de una secuencia autoconsistente de cambios adiabáticos en la paredes de confinamiento. Este mismo procedimiento se puede emplear en el estudio de otras moléculas con estructura piramidal $A B_{3}$, o aquellas en las que se observa que el potencial posee una estructura de doble pozo. 


\section{Capítulo 6}

\section{Conclusiones}

Los sistemas cuánticos confinados tienen una gran importancia, como lo demuestra el gran número de modelos que se han usado en diferentes campos de la física para explicar diferentes fenómenos. Como ya lo hemos mencionado antes, la ecuación de Schrödinger para los SCC no tiene, en general una solución analítica, por lo que se han implementado una serie de métodos que permiten obtener soluciones aproximadas. En este trabajo hemos propuesto un método en el que la función de onda se expresa en forma de una serie de Taylor, lo cual reduce el problema de eigenvalores al de encontrar los ceros de una función. Con este método podemos obtener con una precisión muy alta tanto a los eigenvalores de la energía como las eigenfunciones de los SCC que se pueden reducir a un grado de libertad. La precisión de los resultados de este método se ven limitados solo por la precisión del compilador con el que se trabaja; por ejemplo, los resultados mostrados en los capítulos 2 y 3 se pueden mejorar para reportar más 30 cifras decimales, si se usa el paquete Ultrabasic que permite manejar variables reales de más de 60 dígitos, o bien usando los paquetes de álgebra simbólica como Mathematica, Maple o Derive, en los cuales el usuario define el número de cifras significativas con las que quiere realizar el cálculo.

El oscilador armónico isotrópico confinado es muy interesante por si mismo, pero, su estudio se vuelve más importante debido a que mediante él se pueden estudiar 
problemas más complejos, como por ejemplo el átomo de hidrógeno confinado, con o sin campo magnético.

El método que empleamos para el estudio del OAIC nos permite determinar el estado base, así como los estados excitados, utilizando la misma forma funcional de la función de onda. A diferencia de otros métodos, como el variacional, en el que se necesita utilizar una función de prueba diferente para cada estado. Cuando el oscilador armónico se confina dentro de una caja esférica impenetrable, se rompe la degeneración de los niveles de energía, pero la simetría del problema permanece inalterada. Un hecho importante que hay que mencionar es que al encajonar al oscilador armónico e ir reduciendo el tamaño de la caja, se producen cruces en los niveles de energía y para radios menores, dichos niveles invierten su ordenamiento.

Con el método propuesto se pueden determinar simultáneamente los eigenvalores de la energía y las eigenfunciones; con estas últimas hemos calculado algunos valores esperados de la posición y hemos notado que no se habían reportado antes estas cantidades. Tanto los valores esperados de la posición como los eigenvalores de la energía tienen un comportamiento monótono como función del radio de la caja de confinamiento, además cuando el radio de la caja crece, se recobran los valores del caso libre, como era de esperarse.

Como una aplicación del OAIC se estudió al átomo de hidrógeno confinado en una caja esférica impenetrable, mediante la base del OAIC. Los resultados obtenidos son razonables para el número de funciones base usadas, si se incrementa este número se pueden obtener mejores valores. Sin embargo, debemos de notar que la precisión de este resultado, con el mismo número de funciones base, es casi la misma para diferentes radios de la caja. Lo que no sucedería por ejemplo, si la base usada fuera la de una partícula dentro de una caja del mismo radio, va que al aumentar el tamaño del radio se requerirían de un número cada vez mayor de funciones base para obtener la misma precisión.

En el estudio del átomo de hidrógeno confinado, también hacemos uso del método presentado en la sección 1.2. Mediante este método se obtuvieron los valores más 
precisos reportados en la literatura, tanto para los eigenvalores de la energía, como para otras cantidades físicas.

Como ya lo habiamos mencionado, mediante el mismo método podemos determinar los eigenvalores de la energía del estado base y los estados excitados y simultáneamente las eigenfunciones. Además con el mismo formalismo es posible estudiar el átomo de hidrógeno confinado mediante paredes impenetrables (condiciones de Dirchlet), penetrables y bajo condiciones de Neumann.

En el problema confinado dentro de paredes impenetrables, la degeneración de los niveles de energía presente en el caso libre, se remueve con el confinamiento, aunque la simetría del problema permanece inalterada. Al igual que en el OAIC, al reducir el radio de la caja de confinamiento aparecen cruces en algunos niveles de energía y para radios menores el orden se invierte. Esto puede ser muy importante para la descripción del comportamiento de átomos multielectrónicos sometidos a altas presiones.

Una vez que se obtuvieron los eigenvalores de la energía y eigenfunciones para el estado base, se procedió a calcular algunas propiedades físicas como el desdoblamiento hiperfino, el apantallamiento magnético nuclear, la polarizabilidad y la presión que ejercen las fronteras sobre el átomo de hidrógeno. El comportamiento de los eigenvalores de la energía, los valores esperados de la posición y de las cantidades físicas mencionadas, como función del tamaño de la caja es mónótono.

Como resultado del confinamiento las funciones de onda se ven comprimidas al interior de la caja de radio $r_{0}$, su forma cambia poco, pero el número de nodos de la misma permanece constante. Podemos considerar que la función de onda del estado base para el problema encajonado es prácticamente la del problema libre para un radio mayor o igual a 8 a.u., mientras que para el estado $2 s$ es de alrededor de 20 a.u. y para el $2 p$ es de unos 15 a.u. Así que este radio es en general diferente para cada estado.

En el capitulo 4 estudiamos al átomo de hidrógeno bidimensional confinado mediante paredes circulares impenetrables. En este capitulo decidimos hacer primero el estudio con el método variacional directo, a manera de preparación para 
su posterior aplicación a problemas más complicados y de un mayor número de grados de libertad y finalmente aplicamos el método de las series.

Las energías obtenidas mediante el método variacional directo no son tan precisas como las que se obtienen con el método de las series. Sin embargo, es un método sencillo que proporciona valores de la energía con la precisión suficiente para entender el comportamiento del sistema.

Los eigenvalores de la energía y los valores esperados de $\langle r\rangle,\left\langle r^{2}\right\rangle$ y $\left\langle r^{-1}\right\rangle$ fueron calculados como función del radio del círculo de confinamiento. Encontramos que estos valores siguen un comportamiento monótono y que asintóticamente tienden a los valores libres, como en los casos anteriores, sin embargo, la distancia más probable $r_{\max }$ no sigue un comportamiento monótono para los estados $n=2, l=0$ y $n=2, l=1$.

Usando el método de series encontramos los eigenvalores del problema con una alta precisión. Estos valores pueden servir de referencia para checar la precisión de nuevos métodos.

El electrón en el átomo de hidrógeno bidimensional libre como en el caso confinado, se encuentra más ligado, que su análogo en tres dimensiones. Otra diferencia es que para el problema tridimensional el estado base puede considerarse libre para radios mayores a 8 a.u, mientras que en el caso bidimensional esto sucede alrededor de 4 a.u., la mitad de la distancia que en el caso tridimensional.

En el capítulo 5 volvemos a emplear el método de la sección (1.2) para estudiar los espectros de inversión del $\mathrm{NH}_{3}$ y sus especies isotópicas. La molécula del $\mathrm{NH}_{3}$ tiene forma de una pirámide triangular, con los tres hidrógenos en su base y el átomo de nitrógeno en la arista. La inversión ocurre cuando el átomo de nitrógeno oscila de un lado a otro del plano de los hidrógenos, este es un problema casi tan viejo como la misma mecánica cuántica y ha sido extensamente estudiado experimentalmente y mediante método empíricos, pero poco se ha hecho a través de métodos ab-initio. En este trabajo proponemos que el potencial de doble pozo responsable de la inversión sea un polinomio de cuarto grado, simétrico en la coordenada de inversión $x$. Como los estados más bajos de este potencial 
anarmónico cuártico estan muy cercanos se hace un cálculo de alta precisión para determinar los eigenvalores del doble pozo. El método que nos permite realizar esta operación es el mismo que usamos en los capítulos 2 y 3 . El polinomio de cuarto grado se obtuvo mediante el ajuste de mínimos cuadrados de una superficie de potencial generada por el programa Munich que es un programa usado en química cuántica. Los resultados obtenidos para el $\mathrm{NH}_{3}$ y el $\mathrm{ND}_{3}$ reproducen satisfactoriamente a las constantes moleculares y a los valores experimentales. En el caso del $\mathrm{NH}_{2} \mathrm{D}$ y el $\mathrm{ND}_{2} \mathrm{H}$, no hay manera de saberlo, porque hay poco valores experimentales disponibles, pero podemos anticipar que estos resultados no serán tan buenos, debido a que el proceso de inversión para estas últimas dos moléculas no es simétrico y por lo tanto la fórmula de la masa reducida del sistema debe de ser diferente a la empleada para el estudio del $\mathrm{NH}_{3}$ y $\mathrm{ND}_{3}$.

\section{Aplicaciones futuras}

Al igual que el método variacional directo, pero con mayor precisión se puede aplicar el método de las series aquí presentado, para estudiar otros problemas que puedan reducirse a un grado de libertad. Algunos de los sistemas de interés son: el oscilador anarmónico isotrópico, potenciales (unidimensionales) con pozos múltiples, tanto simétricos como asimétricos, factores de Franck-Condon para el potencial de Morse en moléculas diatómicas, etc.

Generalizando este método a más de un grado de libertad podrían abordarse problemas como el efecto Stark y el Zeeman en el átomo de hidrógeno, el átomo de hidrógeno y excitones en puntos cuánticos confinados en geometrías diferentes a la esférica, así como átomos multielectrónicos confinados en cajas esféricas, excitones en alambres cuánticos y cristalitas, centros F, etc..

En el método de series para $m$ grados de libertad la función de onda estará representada por $\psi\left(x_{1}, x_{2}, x_{3}, \ldots, x_{m}\right)=\sum a_{i j k \ldots q} x_{1}^{i} x_{2}^{j} x_{3}^{k} \ldots x_{m}^{q}$. Como el método esta basado en la evaluación de ésta función en las paredes de la caja, el número de éstas evaluaciónes aumenta rápidamete con el número de grados de libertad del problema. Por lo que una de las investigaciones futuras consistirá en encontrar la manera de implementarlo eficientemente para problemas de más de un grado de libertad. 


\section{Referencias}

[1] A. Michels, J. De Boer and A. Bijl, Physica 4, 981 (1937).

[2] A. Sommerfeld and H. Welker, Ann. Phys. 32, 56 (1938).

[3] S. R. De Groot and C. A. Ten Seldam, Physica 12, 669 (1946).

[4] R. B. Dingle, Proc. Cambridge Philos. Soc. 49, 103 (1953).

[5] E. P. Wigner, Phys. Rev. 94, 77 (1954).

[6] A. D. Buckingham and K. P. Lawley, Mol. Phys. 3, 219 (1960).

[7] K. K. Singh, Proc. Nat. Inst. Science India A 27, 86 (1961).

[8] B. M. Gimarc, J. Chem. Phys. 44, 373 (1966).

[9] B. F. Gray, J. Chem. Phys. 55, 2848 (1971).

[10] V. C. Aguilera-Navarro, W. M. Kloet and A. H. Zimmermann, Rev. Bras. Fis. 1, 55 (1971).

[11] L. S. Cederbaum and K. Schönhammer, Phys. Rev. A 12, 2257 (1975).

[12] D. Suryanarayana and J. A. Weil, J. Chem. Phys. 64, 510 (1976).

[13] E. V. Ludeña, J. Chem. Phys. 66, 468 (1977).

[14] M. Friedman, Y. Rosenfeld, A. Rabinovitch and Thieberger, J. Comp. Phys. 26, 169 (1978).

[15] M. Friedman, A. Rabinovitch and Thieberger, J. Comp. Phys. 33, 359 (1979).

[16] J. A. Weil, J. Chem. Phys. 71, 2803 (1979)

[17] E. Ley-Koo and S. Rubinstein, J. Chem. Phys. 71, 351 (1979).

[18] E. Ley-Koo and S. Rubinstein, J. Chem. Phys. 73, 887 (1980).

[19] J. Fleischauer, Z. Naturforsch 35 a, 1285 (1980).

[20] E. Ley-Koo and S. A. Cruz, J. Chem. Phys. 74, 4603 (1981).

[21] J. Killingbeck, Phys. Lett. 84 A, 95 (1981).

[22] F. M. Fernández and E. A. Castro, J. Math. Phys. 23, 1103 (1982).

[23] F. M. Fernández and E. A. Castro, J. Chem. Phys. 75, 2908 (1981). 
[24] J. L. Marín and S. A. Cruz, Am. J. Phys. 59, 931 (1991).

[25] J. L. Marín and S. A. Cruz, J. Phys. B: At. Mol. Opt. Phys. 24, 2899 (1991).

[26] J. L. Marín and S. A. Cruz, J. Phys. B: At. Mol. Opt. Phys. 25, 1965 (1992).

[27] C. Joslin and S. Goldman, J. Phys. B: At. Mol. Opt. Phys. 25, 1965 (1992).

[28] S. Goldman and C. G. Joslin, J. Phys. Chem. (1992).

[29] N. Aquino, Int. J. Quantum Chem. 54, 107 (1995).

[30] R. Vargas, J. Garza and A. Vela, Phys. Rev. E 53, 1954 (1996).

[31] F. M. Fernández and E. A. Castro, Kinam 4, 193 (1982).

[32] D. S. Kothari and F. C. Auluck, Science and Culture 6, 370 (1940).

[33] F. C. Auluck, Proc. Nat. Inst. Sci. India 7, 133 (1941).

[34] F. C. Auluck, Proc. Nat. Inst. Sci. India 7, 383 (1941).

[35] F. C. Auluck, Proc. Nat. Inst. Sci. India 8, 147 (1942).

[36] S. Chandrasekhar, Astrophys. J. 97, 263 (1943).

[37] F. C. Auluck and D. S. Kothari, Proc. Cambridge Philos. Soc. 41, 175 (1945).

[38] N. Aquino, J. Phys. A: math. Gen. 30, 2403 (1997).

[39] B. Suryan, Phys. Rev 71, 741 (1947).

[40] E. M. Corson and I Kaplan, Phys. Rev. 71, 130 (1947).

[41] R. B. Dingle, Proc. R. Soc. London Ser. A 212, 47 (1952).

[42] J. S. Baijal and K. K. Singh, Prog. Theor. Phys. 14, 214 (1955).

[43] P. Dean, Proc. Cambridge Phil. Soc. 62, 277 (1966).

[44] R. Vawter, Phys. Rev. 174, 749 (1968).

[45] R. Vawter, J. Math. Phys. 14, 1864 (1973).

[46] A. Consortini and B. R. Frieden, Nuovo Cimento 35 B, 153 (1976).

[47] F. C. Rotbar, J. Phys. A: Math. Gen 11, 2363 (1978).

[48] V. C. Aguiler-Navarro, E. Ley-Koo and A. H. Zimermann, J. Phys. A: Math. Gen. 13, 3585 (1980). 
[49] J. L. Marín and S. A. Cruz, Am. J. Phys. 56, 1134 (1988).

[50] F. M. Fernández and E. A. Castro, Kinam, 5, 179 (1983).

[51] T. L. Cotrell, Trans. Faraday Soc. 47, 337 (1951).

[52] C. A. Ten Seldam and S. R. De Groot, Physica 18, 891 (1952).

[53] C. A. Ten Seldam and S. R. De Groot, Physica 18, 905 (1952).

[54] K. K. Singh, Physica 30, 211 (1964).

[55] B. M. Gimarc, J. Chem. Phys. 47, 5110 (1967).

[56] H. Müller, Theor. Chim. Acta (Berlin) 32, 331 (1974).

[57] E. V. Ludeña, J. Chem. Phys. 69, 1770 (1978).

[58] E. V. Ludeña and M. Gregori, J. Chem. Phys. 71, 2235 (1979).

[59] P. L. Kapur and Peierls, Proc. R. Soc. London, Ser A 166, 277 (1938).

[60] A. J. Siegert, Phys. Rev. 56, 750 (1939).

[61] A. Sommerfeld and H. Hartman, Ann. Physik (Leipzig) 37, 333 (1940).

[62] M. F. Osborne, Phys. Rev. 81, 147 (1951).

[63] R. B. Dingle, Phys. Rev. 82, 966 (1951).

[64] S. A. Moszkowski, Phys. Rev. 99, 803 (1955).

[65] L. Eyges, Ann. Phys. 2, 101 (1957).

[66] R. C. Baetzold, C. T. Tahk and D. J. Wilson, J. Chem. Phys. 45, 4209 (1966).

[67] A. Rabinovitch and J. Zak, Phys. Rev. B 4, 2358 (1971).

[68] E. Switkes and E. L. Russell, J. Chem. Phys. 64, 3774 (1976).

[69] T. Lukes, G. A. Ringwood and B. Suprato, Physica 84 A, 421 (1976).

[70] C. H. Maier, L. S. Cederbaum and W. Domcke, J. Phys. B 13, L119 (1980).

[71] J. Killingbeck, Phys. Lett. 78A, 235 (1980).

[72] E. Wigner and F. Seitz, Phys. Rev. 43, 804 (1933).

[73] E. Wigner and F. Seitz, Phys. Rev. 46, 509 (1934).

[74] H. Fröhlich, Phys. Rev. 54, 945 (1938). 
[75] R. Kronig, J. de Boer and J. Korringa, Physica 12, 245 (1946).

[76] J. Bardeen, J. Chem. Phys. 6, 372 (1938).

[77] E. Wigner and H. B. Huntington, J. Chem. Phys. 3, 764 (1935).

[78] G. Campoy y A. Palma, Acta Méx. de Ciencia y Tecnología 11 (8), 47 (1984).

[79] G. Campoy, Ph. D. Tesis, México (1990), sin publicar.

[80] A. Palma and G. Campoy, Phys. Lett. A 21, 221 (1987).

[81] R. N. Chaudhuri and B. Mukherjee, J. Phys. A: Math. Gen. 17, 277 (1984).

[82] J. F. Rivas-Silva, G. Campoy and A. Palma, Int. J. Quantum Chem. 43, 747 (1992).

[83] G. Campoy, A. Palma and L. Sandoval, Int. J. Quantum Chem.: Quantum Chemistry Symposium 23, 355 (1989).

[84] N. Aquino, A. Palma, J. L. López, and M. A. Rosales, Pramana J. Phys. 45, 75 (1995).

[85] A. S. Davydov, Theory of Molecular Excitons (Plenum, New York, 1971) Cap. 1.

[86] S. V. Nair, S. Sinha, and K. C. Rustagi, Phys. Rev. B 35, 4098 (1987).

[87] P. E. Lippens and M. Lannoo, Phys. Rev. B 41, 6079 (1990).

[88] G. T. Einevoll, Phys. Rev. B.45, 3410 (1992).

[89] Y. Kayanuma, Phys. Rev. B 38, 9797 (1988).

[90] Y. Kayanuma and H. Momiji, Phys. Rev. B41, 10261 (1990).

[91] N. Porras-Montenegro and S. T. Pérez-Merchancano, Phys. Rev. B 46, 9780 (1992).

[92] W. Jaskólski, Phys. Rep. 271, 1 (1996).

[93] F. Rajadell, J. Planelles, W. Jaskólski and C. Zicovich-Wilson, Int. J. Quantum Chem. 60, 993 (1996).

[94] F. M. Fernández and E. A. Castro, Phys. Rev. A 24, 2883 (1981).

[95] W. H. Press, B. P. Flannery, S. A. Teukolsky and W. T. Vetterling Numerical Recipes: The Art of Scientific Computing (New York: Cambridge University Press, 1986). 
[96] W. S. Dorn and D. D. MacCraken, Numerical Methods with Fortran IV:Case Studies (New York: Wiley, 1972).

[97] J. P. Killinbeck Microcomputer Algorithms, Action from Algebra (New York:Hilger, 1991).

[98] J. L. Marín, Tesis Doctoral UNAM, México 1992.

[99] F. M. Fernández and E. A. Castro, J. Phys. A:Math and Gen. 14, L485 (1981).

[100] O. L. de Lange and R. E. Raab ,Operator Methods in Quantum Mechanics (Oxford: Claredon, 1991).

[101] M. Moshinsky, The Harmonic Oscillator in Modern Physics: From Atoms to Quarks (London: Gordon and Breach 1969).

[102] E. Ley-Koo, S. Mateos-Cortes, and G. Villa-Torres, Int. J. Quantum Chem. 50, 175 (1995).

[103] E. Ley-Koo, S. Mateos-Cortes, and G. Villa-Torres, Int. J. Quantum Chem. 58, 23 (1996).

[104] A. Chodos, R. Jaffe, K. Johnson, C. Thorn, and V. F. Weiskopf, Phys. Rev. 29, 3471 (1974).

[105] V. F. Weiskopf, in Lepton and Hadron Structure, Subnuclear Series, edited by A. Zichichi (Academic, New York, 1974), Vol. 12, p. 777.

[106] J. L. Marín, A. Clark, R. Rodríguez, R. Aceves, and S. A. Cruz, J. Chem. Phys. 76, 3107 (1982).

[107] E. Castaño y G. Kirczenow, Solid State Comm. 70, 801 (1989), Phys. Rev. B41, 5055 (1990).

[108] L. D. Landau y E. Lifshitz, Mecánica Cuántica, Teoria no-relativista (Reverte, México 1976), L. I. Shiff, Quantum Mechanics (McGraw-Hill, New York 19868).

[109] L. E. Brus, J. Chem. Phys. 79, 5566 (1983).

[110] L. Katsikas, A. Eychmüller, M. Giersing and H. Weller, Chem. Phys. Lett. 172, 201 (1990).

[111] J. Gorecki and W. Byers Brown, J. Phys. B: At. Mol. Opt. Phys. 20, 5953 (1987), 21, 403 (1988).

[112] J. Gorecki and W. Byers Brown, J. Chem. Phys. 89, 2138 (1988).

[113] T. L. Cotrell and S. Paterson, Phil. Mag. 42, 391 (1951). 
[114] W. Byers Brown, Proc. Cambridge Phil. Soc. 58, 251 (1958).

[115] N. Argydes, Phys. Rev. 154, 410 (1967).

[116] F. M. Fernández and E. A. Castro, J. Chem. Phys. 75, 2908 (1981).

[117] T. Pang, Phys. Rev. A, 49, 1709 (1994).

[118] J. Killinbeck, Phys. Lett. 84 A, 95 (1981).

[119] M. Friedman, A. Rabinovitch and R. Thieberger, J. Comput. Phys. 33, 359 (1979).

[120] R. Loudon, Am. J. Phys. 27, 649 (1959).

[121] M. Andrews, Am. J. Phys. 34, 1194 (1966).

[122] L. K. Haines and D. H. Roberts, Am. J. Phys. 37, 1145 (1969).

[123] J. F. Gomes and A. H. Zimerman, Am. J. Phys. 48, 579 (1980).

[124] I. R. Lapidus, Am. J. Phys. 49, 807 (1981).

[125] H. N. Nuñez Yepez, C. A. Vargas, and A. L. Salas-Brito, Eur. J. Phys. 8, 189 (1987).

[126] G. Q. Hassoun, Am J. Phys. 49, 143 (1981).

[127] J. W.-K. Huang and A. Kozycki, Am. J. Phys. 47, 1005 (1979).

[128] T.-I. Shibuya and C. E. Wulfman, Am. J. Phys. 33, 570 (1965).

[129] B. Zaslow and M. E. Zandler, Am. J. Phys. 35, 1118 (1967).

[130] B. B. Dasgupta, Am. J. Phys. 49, 189 (1981).

[131] M. M. Nieto, Am. J. Phys. 47, 1067 (1979).

[132] K. Andrew, Am. J. Phys. 58, 1177 (1990).

[133] W. Kohn and J. M. Luttinger, Phys. Rev 98, 915 (1955).

[134] A. Cisneros and H. McIntosh, J. Math. Phys. 10, 277 (1969).

[135] X. L. Yang, S. H. Guo, F. T. Chan, K. W. Wong, and W. Y. Ching, Phys. Rev. A, 43, 1186 (1991).

[136] J. Gorecki and W. Beyers Brown, J. Phys. B: At. Mol. Opt. Phys. 22, 2659 (1989). 
[137] H. N. Spector and J. Lee, Am. J. Phys. 53, 248 (1985).

[138] X. G. Wen and A. Zee, Phys. Rev. Lett. 62, 1937 (1989).

[139] K. W. Wong and W. Y. Ching, Physica B+C 158C,1 (1989); 158C, 15 (1989).

[140] B. D. Perlson and J. A. Weil, J. Magn. Reson. 15, 594 (1974).

[141] H. Y. Sheng, E. F. Barker and D. M. Dennison, Phys. Rev. 60,786 (1941).

[142] M. V. Migeotte and E. F. Barker, Phys. Rev. 50, 418 (1936).

[143] F. T. Wall and G. Glocker, J. Chem. Phys. 5, 314 (1937).

[144] W. E. Good, Phys. Rev. 70, 213 (1946).

[145] W. S. Benedict and E. K. Plyer, Can. J. Phys. 35, 1235 (1957).

[146] C. W. Townes, Phys. Rev. 70, 665 (1946).

[147] M. T. Weiss and M. W. P. Strandberg, Phys. Rev. 83, 567 (1951).

[148] M. F. Manning, J. Chem. Phys. 3, 136 (1935).

[149] R R Newton and L H Thomas, J. Chem. Phys. 16, 310 (1948).

[150] J. D. Swalen and J. A. Ibers, J. Chem. Phys. 36, 1914 (1962).

[151] C. H. Townes and A. L. Schalow, Microwave Spectroscopy (Dover, New York, 1975) p. 300.

[152] C. C. Costain and G. B. B. M. Shuterland, J. Phys. Chem. 65, 321 (1952).

[153] P. R. Bunker, W. P. Kraemer and V. Spirko, Can. J. Phys. 62, 1801(1984)

[154] N. Aquino, Tesis de Maestría, UNAM, México (1992).

[155] K. Banerjee and S. P. Bhatnagar, Phys. Rev. D18, 4767 (1978)

[156] J. L. Marín, R. Rosas and A. Uribe, Am. J. Phys. 63, 460 (1995).

[157] M. T. Weiss and M. W. Strandberg, Phys. Rev. 83, 567 (1951)

[158] N. Aquino, J. Math. Chem. 18, 349 (1995). 